\title{
Akustische Beeinflussung einer Instabilität in Kanälen mit überströmten Resonatoren
}

\author{
Dissertation \\ zur Erlangung des Doktorgrades \\ der Mathematisch-Naturwissenschaftlichen Fakultäten \\ der Georg-August-Universität zu Göttingen \\ vorgelegt von \\ Matthias Jüschke \\ aus \\ Oldenburg
}

Göttingen 2006 
D7

Referent: Prof. Dr. Dirk Ronneberger

Korreferent: Prof. Dr. Ulrich Parlitz

Tag der mündlichen Prüfung: 18. Juli 2006 


\section{Inhaltsverzeichnis}

1 Einleitung 1

2 Eigenschaften runder Resonatorabschnitte 4

2.1 Aufbau des Kanals . . . . . . . . . . . . . . . . . . . . . . 4

2.2 Resonatorabschnitt ............................ 5

2.2.1 Akustische Admittanz der Wand im Resonatorabschnitt . . . . 7

2.3 Verhalten bei Strömung . . . . . . . . . . . . . . . . . . . 9

2.3.1 Spektren der Druckschwankungen . . . . . . . . . . . . . . . 9

2.3 .2 Einfluss der Resonatorenzahl . . . . . . . . . . . . . . . . . . . . 12

2.3.3 Einfluss der Querschnittsgeometrie . . . . . . . . . . . . . 14

2.3.4 Amplitudenstatistik der Druckschwankungen . . . . . . . . . . . 16

2.4 Druckamplituden bei Beschallung . . . . . . . . . . . . . . . . . 17

2.4.1 Die Amplitudenabhängigkeit der Streufaktoren . . . . . . . . . . 17

2.4 .2 Frequenz- und Machzahlabhängigkeit von $t^{+} \ldots \ldots . . . .23$

2.4.3 Spektren bei monofrequenter Beschallung . . . . . . . . . . . . . 27

2.5 Schallinduzierte Veränderung des Druckabfalls . . . . . . . . . . . . . . 32

2.5 .1 Strömungszustand $\mathrm{im}$ Rohr . . . . . . . . . . . . . . . 33

2.5.2 Druckabfall am Resonatorabschnitt ohne Beschallung . . . . . . 35

2.5.3 Einfluss von Schall auf den Druckabfall . . . . . . . . . . . . . 39

2.6 Höhere akustische Moden . . . . . . . . . . . . . . . . . . . 42

2.6.1 Druckschwankungen in der letzten Resonatorkammer . . . . . . 42

2.6.2 Einfluss externer Beschallung . . . . . . . . . . . . . . . 45

2.6.3 Einfluss der ersten höheren Mode auf den Druck . . . . . . . . . 48

3 Eigenschaften des rechteckigen Resonatorabschnitts 54

3.1 Aufbau des Resonatorabschnitts . . . . . . . . . . . . . . . . . 54

3.2 Verhalten bei Strömung . . . . . . . . . . . . . . . . . . . . 57

3.2 .1 Spektrales Verhalten . . . . . . . . . . . . . . . . 57

3.2.2 Amplitudenstatistik der Druckschwankungen . . . . . . . . . . 58 
3.3 Einfluss von Schall auf den Druckabfall . . . . . . . . . . . . . . . . . . 58

3.4 Transmissionsfaktoren ...................... 61

4 Theorie zur Schallausbreitung im Resonatorabschnitt 65

4.1 Aufstellen der Dispersionsrelation . . . . . . . . . . . . . . 65

4.1 .1 Rechteckprofil . . . . . . . . . . . . . . . . . 66

$4.1 .2 \quad$ Strömungsprofil . . . . . . . . . . . . . . . . . . . . 69

4.2 Das Problem der Ausbreitungsrichtung der Moden . . . . . . . . . . . . 70

4.2.1 Ausbreitungsrichtung in Vorgängerarbeiten . . . . . . . . . . . 70

4.2.2 Weitere Hinweise zur Ausbreitungsrichtung . . . . . . . . . . . . 74

4.3 Zusammenfassung der Modenausbreitung . . . . . . . . . . . . . . . . . 82

4.3.1 Alternativer Ansatz . . . . . . . . . . . . . . . . . . 83

5 Schlussbetrachtungen $\quad 85$

5.1 Ergebnisse . . . . . . . . . . . . . . . . . . . . . . . 85

5.2 Ausblick . . . . . . . . . . . . . . . . . . . . . 87

$\begin{array}{lr}\text { A Ansteuerungsparameter } & \mathbf{8 8}\end{array}$ 


\section{Symbolverzeichnis}

$A_{s} \quad$ Amplitude der Lautsprecheranregung

$B \quad$ Breite der Resonatorkammern (rund: $5 \mathrm{~mm}$, rechteckig: $4 \mathrm{~mm}$ )

c Schallgeschwindigkeit

$D \quad=50 \mathrm{~mm}$, Rohrdurchmesser

$e \quad$ Konstante der auf die Dichte bezogenen Gesamtenthalpie

$f_{\max } \quad$ Frequenz maximaler Schallverstärkung

$f_{s} \quad$ Frequenz der Anregung

$i \quad$ imaginäre Einheit

$J_{m} \quad$ Besselfunktion

$k \quad$ Betrag der Wellenzahl

$k_{r} \quad$ radiale Wellenzahl im Rohr

$k_{r, \mathrm{~W}}$ radiale Wellenzahl in den Resonatorkammern

$k_{x} \quad$ axiale Wellenzahl

$L \quad$ Länge der offnen Kammern im Resonatroabschnitt

$m=\rho u$, Konstante der Kontinuitätsgleichung, sowie azimutale Mode $\exp (i m \phi)$

Ma über den Kanalquerschnitt gemittelte Machzahl

$\mathrm{Ma}_{\text {ref }}=0,96 \sqrt{\Delta p_{1} / p_{0}}$, ungefähre Machzahl

$p \quad$ statischer Druck

akustischer Wechseldruck

$p_{0} \quad$ statischer Druck im Labor

$p_{\text {dyn }} \quad$ Staudruck

$\operatorname{Pr} \quad=0,713$, Prandtlzahl von Luft

$r \quad$ radiale Koordinate

$R \quad=D / 2=25 \mathrm{~mm}$, Rohrradius

$r^{+} \quad$ Reflexionsfaktor bei Schalleinfall in Strömungsrichtung 
$R_{a} \quad=100 \mathrm{~mm}$, Außenradius des Resonators

$S \quad$ Breite des freien Ringspalts im Rohr mit Zentralkörper

$s^{+} \quad \approx t^{+} / r^{+}$, Amplitudenverhältnis der stromab laufenden zur stromauf laufenden Welle bei der Beschallung aus dem Resonator

$t^{+} \quad$ Transmissionsfaktor in Strömungsrichtung

$\bar{u} \quad$ Strömungsgeschwindigkeit

$u_{r} \quad$ Radialschnelle

$u_{x} \quad$ Axialschnelle

$u_{\phi} \quad$ azimutale Schnelle

$w_{i} \quad$ gespamelte Mikrofonwerte

$Y \quad$ Feldadmittanz

$Y_{m} \quad$ Neumannfunktion

$Y_{\mathrm{W}} \quad$ Wandadmittanz

$\alpha \quad$ Winkel zwischen Mikrofonen, die im Resonator eingebaut sind

$\gamma \quad=1,401$, Adiabatenexponent von Luft

$\gamma_{W_{1} W_{2}}$ Kohärenz von Mikrofonsignalen

$\delta=\sqrt{2 \nu / \omega}$, Stokesdicke

$\Delta p \quad$ Durckabfall durch den Resonatorabschnitt

$\Delta p_{1} \quad$ Druckabfall am Kanaleinlauf

$\Delta p_{\mathrm{ak}}$ akustisch induzierter Durckabfall

$\eta \quad=\frac{i}{\omega^{\prime}} u_{r}$, Auslenkung

$\lambda \quad$ Rohrreibungskoeffizient

$\mu \quad$ dynamische Zähigkeit von Luft

$\nu \quad$ kinematische Zähigkeit von Luft

$\rho \quad$ Dichte der Luft

$\phi_{L} \quad$ Phasenunterschied, mit dem die Lautsprecher am Resonator betrieben werden

$\omega \quad$ Kreisfrequenz

$\omega^{\prime} \quad=\omega-k \bar{u}$, dopplerverschobene Kreisfrequenz 


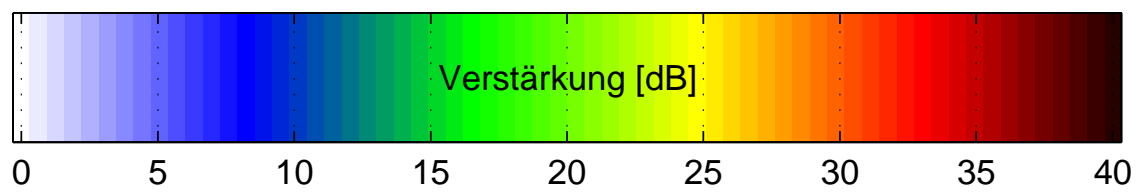

Abb. 0.1: Farbcodierung der spektralen Überhöhungen über den Median des Spektrums

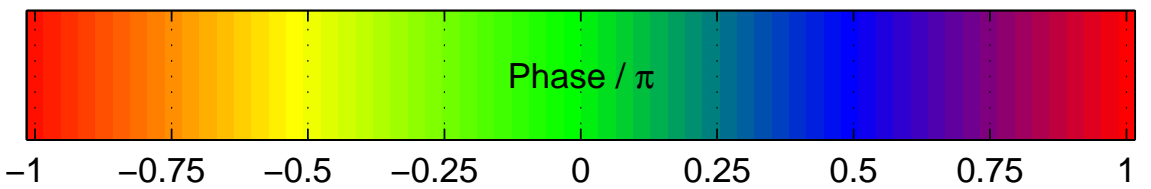

Abb. 0.2: Farbcodierung der Phase 



\section{Kapitel 1}

\section{Einleitung}

In durchströmten Kanälen breitet sich neben der Strömung oft auch Schall aus, beispielsweise in Abgaskanälen von Verbrennungsmotoren oder in Belüftungsanlagen. Häufig ist dieser Schall unerwünscht. Mit dem Ziel, die Schallausbreitung zu dämpfen, können Resonatoren an den Seitenwänden des Kanals angebracht werden. Solche Konfigurationen können bei ruhender Luft die Schalltransmission beträchtlich verringern. Jedoch kann es bei einem durchströmten Kanal zu Schallverstärkung und zu selbsterregten Schwingungen kommen.

Um das akustische Verhalten von derartigen Anordnungen vorhersagen zu können, wurden theoretische Modelle entwickelt, die von einem ebenen Strömungsprofil mit unendlich dünner Wandgrenzschicht ausgehen und die akustisch ausgekleidete Wand als homogen und lokal reagierend mit gegebener Wandadmittanz betrachten (Koch und Möhrung [13], Quinn und Howe [18]). Dabei ist jedoch nicht klar, wie die Randbedingung an dem sprunghaften Übergang zwischen dem hartwandigen Rohr mit der Admittanz Null und dem Abschnitt mit von Null verschiedener Admittanz zu wählen ist.

Die Wahl der richtigen Randbedingung sollte durch Vergleich der Modellvorhersagen mit geeigneten experimentellen Ergebnissen gefunden werden. Das Experiment wurde wegen der übersichtlicheren Strömungsverhältnisse in einem Kanal mit rundem Querschnitt durchgeführt. Die Wandadmittanz sollte möglichst dämpfungsarm und gut zu berechnen sein. Als Wandauskleidung wurde eine Serie scheibenförmiger Hohlräume gewählt, die den Kanal konzentrisch umgeben und nur durch ein feinmaschiges Drahtgitter vom Kanalinneren getrennt sind (siehe Abb. 2.2). Zum Vergleich mit den Modellvorhersagen sollten die vier akustischen Streufaktoren für die Grundmode an einem kurzen, derartig aufgebauten Resonatorabschnitt herangezogen werden. Es zeigte sich allerdings, dass die Wellenausbreitung in dem Resonatorabschnitt facettenreicher ist als ihre Beschreibung durch die erwähnten Modelle, so dass selbst ein qualitativer 
Vergleich kaum möglich war (Förster [9], Rebel [19]).

Zu den zunächst nicht erwarteten Phänomenen gehörte die Beobachtung, dass in einem gewissen Frequenzbereich etwas oberhalb der Resonanzfrequenz der Resonatorkammern Transmissionsfaktoren für die in Strömungsrichtung einfallende Schallwelle auftreten, die weit über eins liegen. Diese Schallverstärkung geht mit einem erhöhten Druckabfall in der Gleichströmung einher (Krause [14], Brandes [2]). Diesen Effekt haben Brandes [2], Lange [15] sowie Lange und Ronneberger [16] genutzt, um durch eine Modulation des verstärkten Schalls eine entsprechende Modulation des Druckabfalls zu erzielen. Auf Grund des oszillierenden Druckabfalls wirkt der Resonatorabschnitt wie eine Dipolquelle, die Schall bei der Modulationsfrequenz abstrahlt. Lange hat auf diese Weise tieffrequenten Schall, der sich im Strömungskanal ausbreitet, breitbandig kompensiert.

In dem Kompensationsexperiment mit einem Rohrdurchmesser von $50 \mathrm{~mm}$ und einer Resonanzfrequenz der Resonatorscheiben von $840 \mathrm{~Hz}$ lag die höchste Modulationsfrequenz bei etwa $200 \mathrm{~Hz}$. Damit eröffnet sich die Möglichkeit, ein akustisch gesteuertes Ventil mit kurzer Ansprechzeit zu konstruieren. Allerdings wird bei der bisher untersuchten Anordnung der Steuerschall verstärkt abgestrahlt, Transmissionsfaktoren bis zu 30 wurden gemessen. Da dieser Schall störend sein kann, liegt es nahe, nach ähnlichen Anordnungen zu suchen, bei denen die Steuerbarkeit des Druckgradienten erhalten bleibt, die starke Schallabstrahlung aber unterdrückt wird. Dieses ist der Ausgangspunkt der vorliegenden Arbeit.

Die bisherigen Beobachtungen [2], [14], [16] lassen sich durch die Hypothese erklären, dass die Strömung in dem Resonatorabschnitt konvektiv instabil ist, diese Instabilität am stromauf liegenden Ende des Abschnitts durch die aufgeprägte Schallwelle angeregt wird und zu einer räumlich anwachsenden Instabilitätswelle führt. Am stromab liegenden Ende des Abschnitts wird ein Teil der stark angewachsenen Strömungsoszillation als Schall abgestrahlt. Außerdem treten nichtlineare Effekte auf, die unter anderem dazu führen, dass der axiale Impuls verstärkt zur Wand transportiert wird (vergleichbar mit dem turbulenten Impulstransport), es kommt zur Erhöhung der Wandschubspannung und des Druckabfalls im Resonatorabschnitt.

Bisher ist es allerdings kaum gelungen, diese Hypothese theoretisch zu untermauern (Großer [10], Rienstra [22]). Zwar wurden innerhalb der bisher untersuchten Modelle stromab anwachsende Moden gefunden, deren komplexe Wellenzahlen hängen aber gänzlich anders von der Frequenz und der Strömungsgeschwindigkeit ab, als man es aufgrund der gemessenen Transmissionsfaktoren erwartet. Bei der neuerlichen Modellierung des Streuprozesses, bei dem noch immer die Randbedingungen am Einlauf und am Auslauf des Resonatorabschnitts geraten werden mussten, wurde zwar eine 
qualitativ bessere, aber noch keineswegs befriedigende Übereinstimmung mit den Beobachtungen erzielt.

Die vorliegende Arbeit ist überwiegend experimentell ausgerichtet. In erster Linie werden weitere Kanalquerschnittsformen und weitere Geometrien zur Anregung der hypothetischen Instabilität untersucht, insbesondere auch solche, bei denen die Abstrahlung einer verstärkten Schallwelle unterdrückt ist. Das gelingt durch die Anregung höherer Moden, die im hartwandigen Kanal nicht ausbreitungsfähig sind.

In den Kapiteln 2 und 3 werden Untersuchungen an Kanälen mit rundem und ringförmigem, sowie mit rechteckigem Querschnitt dargestellt: Zunächst werden Spektren der turbulenten Druckschwankungen als Funktion der Strömungsgeschwindigkeit betrachtet, in denen sich mögliche Instabilitäten durch eine erhöhte spektrale Leistungsdichte bemerkbar machen. Sie sind Hinweise für die dann folgenden Untersuchungen mit Schallanregung. Hierbei werden die Amplitude der stromab abgestrahlten Schallwelle und die Erhöhung des Druckabfalls als Funktion der Anregungsamplitude, der Frequenz und der Strömungsgeschwindigkeit gemessen. Teilweise sind die Abhängigkeiten mit zwei unterschiedlichen Anregungsarten durchgeführt, die Instabilität konnte einerseits als mit der Strömung laufende Grundmode im Kanal, andererseits an der stromauf liegenden Seite des Resonatorabschnitts angeregt werden. Die nichtlinearen Effekte machen sich nicht nur durch Sättigung der abgestrahlten Schallamplitude und durch die Erhöhung des Strömungswiderstandes bemerkbar, sondern auch durch Veränderung der turbulenten Wechseldruckspektren, die ebenfalls betrachtet werden. Mit diesen Messungen soll eine breitere Datenbasis geschaffen werden, mit der zukünftige Modelle verglichen werden können. Darüber hinaus werden Vorgaben für die praktische Anwendung der schallgesteuerten Druckänderung geliefert.

In Kapitel 4 schließen sich theoretische Untersuchung zur Wellenausbreitung in Kanälen mit nachgiebigen Wänden an. Die Frage, in welche Richtung sich die einzelnen Moden ausbreiten, bereitet unerwartete Schwierigkeiten, die hier eingehend betrachtet werden. 


\section{Kapitel 2}

\section{Eigenschaften runder Resonatorabschnitte}

\subsection{Aufbau des Kanals}

Die Messungen wurden in einem Strömungskanal mit kreisförmigem Querschnitt durchgeführt. Dieser Kanal wurde im Rahmen der Diplomarbeiten von Brandes [1] und Enghardt [8] aufgebaut, er ist für die Messung von akustischen Streufaktoren optimiert. In den angeführten Arbeiten ist das Verfahren zur Bestimmung der Streufaktoren näher beschrieben. In Abb. 2.1 ist der Aufbau skizziert.

Die Temperatur und die relative Luftfeuchtigkeit der Laborluft, sowie der Umgebungsdruck werden außerhalb des Kanals gemessen. Am Einlauf befindet sich hinter einem Strömungsgleichrichter ein reflexionsarmer Abschluss, in dem das Rohr auf einer Länge

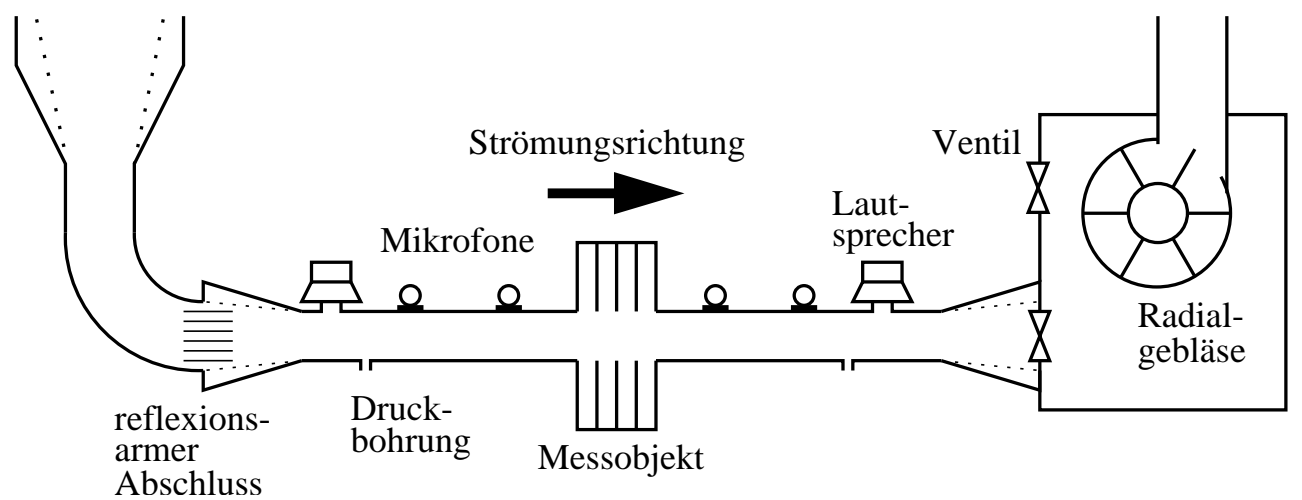

Abb. 2.1: Aufbau des Strömungskanals. 
von einem Meter seinen Durchmesser von $100 \mathrm{~mm}$ auf den Durchmesser des Messrohres, $D=50 \mathrm{~mm}$ verengt. Die erste Druckmessstelle liegt ca. $22 D$ weiter stromab. Verschiedene Untersuchungen haben unterschiedliche Einlauflängen ergeben, nach denen sich ein ausgebildetes turbulentes Strömungsprofil eingestellt hat, sie liegen zwischen $25 D$ und 100D [24]. In der vorliegenden Arbeit wird davon ausgegangen, dass sich schon nach $22 D$ eine weitere Änderung des Strömungsprofils nicht auf die Schallausbreitung und den Druckabfall auswirkt.

Die Strömungsgeschwindigkeit im ersten Teilrohr wird über den Druckabfall am Einlauf (mit Druckwandler vom Typ Setra 239) bestimmt, genau beschrieben wird der Zusammenhang in Abschnitt 2.5.1. Der Strömungszustand im zweiten Teilrohr wird durch eine weitere Druckmessung ermittelt.

Das zu untersuchende Messobjekt wird zwischen den beiden Teilrohren befestigt. Mit einem Rohrdurchmesser von $50 \mathrm{~mm}$ sind bis zu Frequenzen von ca. $4 \mathrm{kHz}$ nur ebene Moden ausbreitungsfähig. Der Schall zur Messung der Streufaktoren wird über zwei Lautsprecher (ALTEC Modell 290-4G) an den Außenseiten der Teilrohre ein-

gebracht. Die Lautsprecher werden über einen Konstant-Strom-Leistungsverstärker (Rohrer-Müchen, 3xPA150-B, $5 \mathrm{kHz}$ ) angesteuert.

Je Teilrohr erfassen bis zu 16 Mikrofone das Schallfeld. Die in dieser Arbeit gezeigten Druckspektren wurden mit 1/4 Zoll Kondensatormikrofonen (Brüel \& Kjær, Typ 4136) aufgenommen.

Die Laborluft wird durch das Rohr über ein Radialgebläse angesaugt. Die Strömungsgeschwindigkeit kann mittels zweier über Schrittmotoren angesteuerter Ventile eingestellt werden. In der Ebene des Messobjekts, in der die Teilrohre zusammentreffen, beträgt die über den Rohrquerschnitt gemittelte Strömungsgeschwindigkeit maximal ca. $115 \mathrm{~m} / \mathrm{s}$.

In der Abbildung 2.1 ist die Strömung konventionsgemäß nach rechts laufend eingezeichnet. Von dieser Konvention wird später bei einigen Bezeichnungen Gebrauch gemacht.

\subsection{Resonatorabschnitt}

Es werden unterschiedliche Resonatorabschnitte untersucht. Den Effekten um die Resonanzfrequenz der Kammern wird besondere Aufmerksamkeit zuteil. Dieses Kapitel beschäftigt sich mit kreisförmigen Resonatoren, in Kapitel 3 wird ein Resonatorabschnitt im rechteckigen Kanal untersucht. 


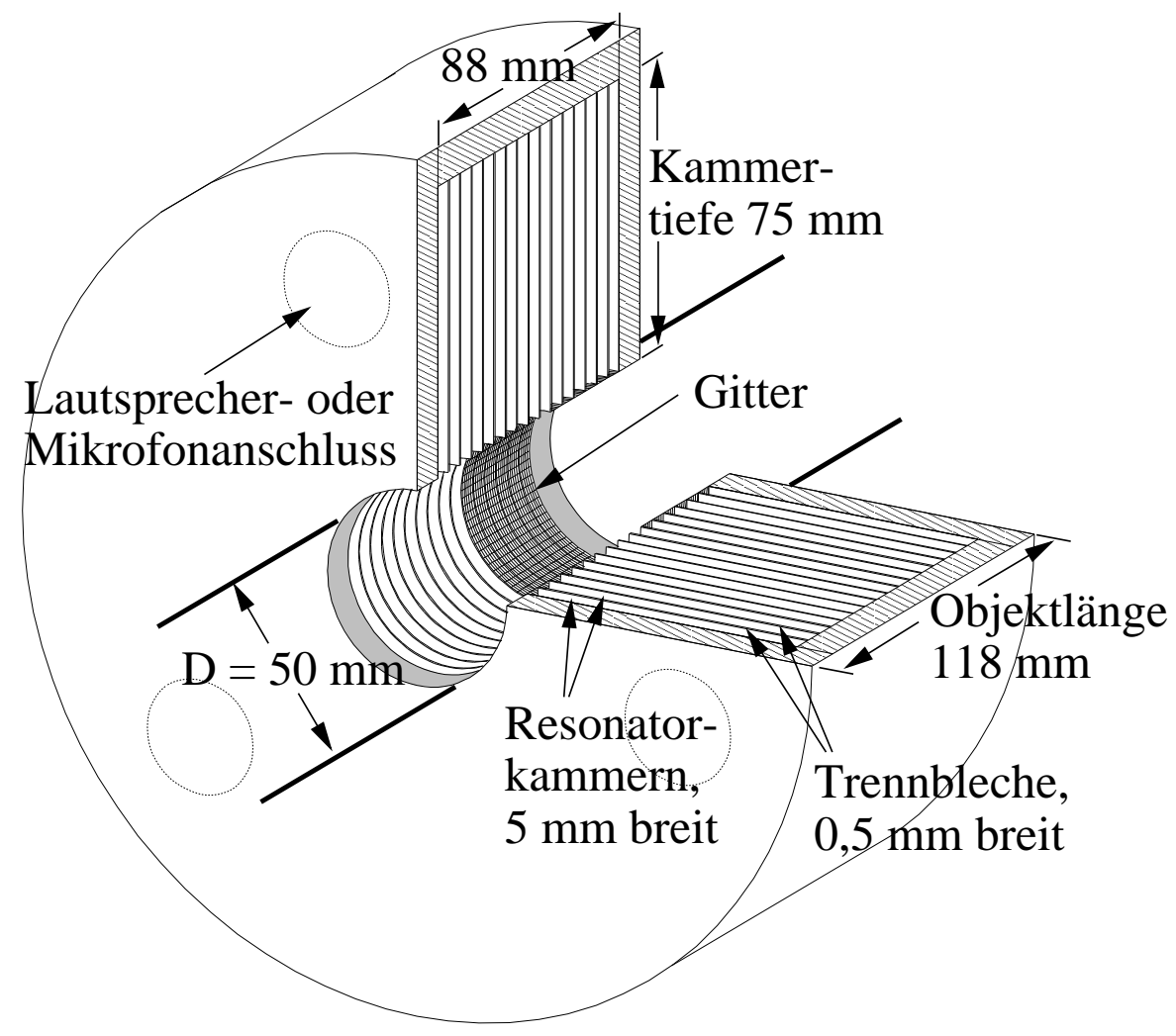

Abb. 2.2: Aufbau des runden Resonatorabschnitts.

Der runde Resonatorabschnitt (siehe Abb. 2.2) besteht aus 16 Resonatorkammern, die jeweils eine Breite von $B=5 \mathrm{~mm}$ haben und durch $0,5 \mathrm{~mm}$ dicke Bleche voneinander getrennt sind. Die Kammern sind $75 \mathrm{~mm}$ tief. Eine Unterteilung in Umfangsrichtung besteht nicht. Der Durchmesser des freien Querschnitts beträgt $50 \mathrm{~mm}$, ist also gleich dem des angeschlossenen Messrohrs. Die Resonatorkammern sind durch ein feinmaschiges Drahtgitter (mit einer Maschenweite von $125 \mu \mathrm{m}$ und einem Drahtdurchmesser von $80 \mu \mathrm{m}$ [2]) vom Rohr getrennt, so dass die turbulenten Fluktuationen der Strömung möglichst wenig in die Kammern eindringen und mit den Trennblechen wechselwirken.

Bei Messungen mit einer kleineren Zahl von Resonatoren sind einige Kammern der stromauf liegenden Seite mit Klebeband abgeklebt. Eine Vergleichsmessung, bei der die abgeklebten Kammern zusätzlich mit einem Messingrohr mit einer Wandstärke von 0,5 mm verdeckt wurden, zeigt keine signifikanten Unterschiede. Eine Abdeckung mit dem Klebeband ist also ausreichend, um die akustische Wirkung der Kammern auszuschalten.

Eine Änderung des Rohrquerschnitts ist ebenfalls möglich. In den Resonatorabschnitt 


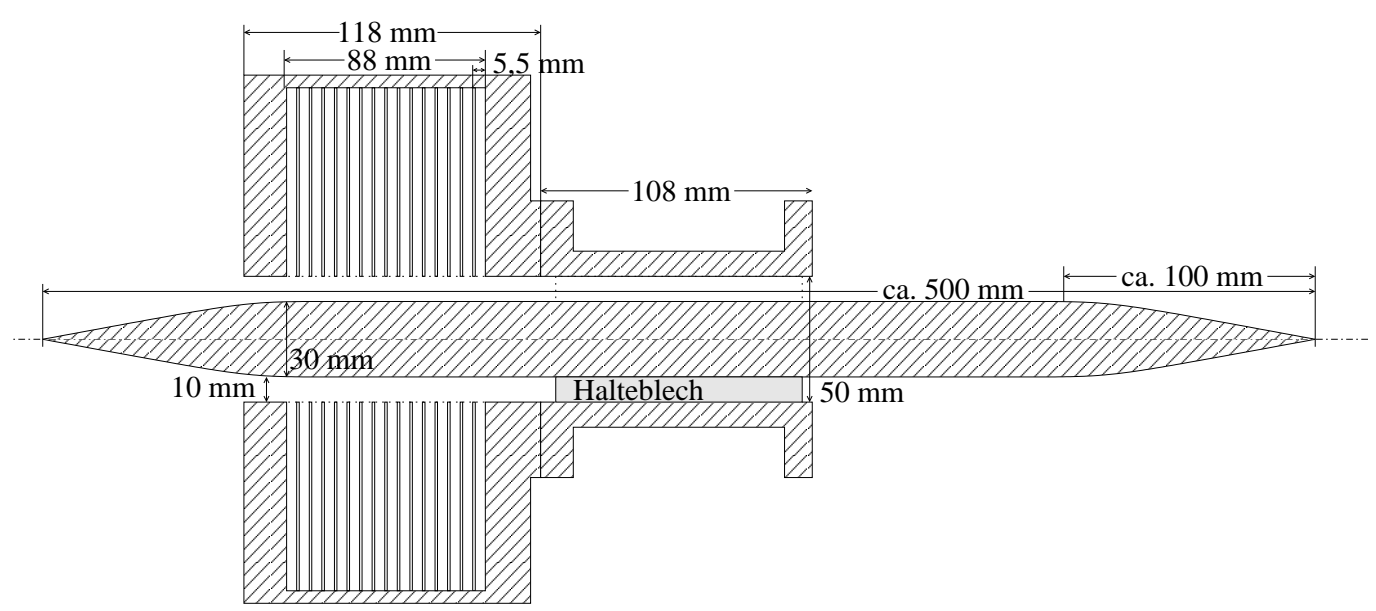

Abb. 2.3: Resonatorabschnitt mit Zentralkörper zur Reduzierung des Rohrquerschnitts.

wird ein Zentralkörper gebracht, so dass ein freier Ringspalt offen bleibt, s. Abb. 2.3. Diese Anordnungen werden über die Breite des freien Spaltes $S$ beschrieben. In der Abbildung ist $S=10 \mathrm{~mm}$ gezeigt. Zwei schmalere Spalte, $S=8 \mathrm{~mm}$ und $5 \mathrm{~mm}$, werden durch Aufsetzen eines Rohrstücks auf den gezeigten Zentralkörper realisiert. Diese Rohrstücke schließen stromauf glatt mit dem Zentralkörper ab. Ein weiterer, kleinerer Zentralkörper lässt einen Spalt von $S=12,5 \mathrm{~mm}$ offen.

Auf einer Seite des Resonatorabschnitts sind im Abstand von $120^{\circ}$ drei Anschlüsse vorhanden. Wahlweise können dort mit dem Anschluss von Lautsprechern (Monacor KU-100) Schallfelder angeregt oder mit Mikrofonen Druckschwankungen aufgenommen werden. Auf der anderen Seite des Resonatorabschnitts sind zwei Mikrofonanbohrungen vorhanden, die $180^{\circ}$ auseinander liegen.

\subsubsection{Akustische Admittanz der Wand im Resonatorabschnitt}

Mit den linearisierten Eulergleichungen in Zylinderkoordinaten (siehe Kap. 4) kann die Admittanz $Y_{\mathrm{W}}=u_{r} / p$ der Resonatoren an der Rohrwand bei $R=25 \mathrm{~mm}$ berechnet werden. Als Randbedingung wird angenommen, dass die Radialschnelle an der AuBenseite der Resonatoren beim Radius $R_{a}=100 \mathrm{~mm}$ verschwindet, $u_{r}\left(R_{a}\right)=0$. Die Axialschnelle ist im Resonator ebenfalls Null, $u_{x}(r)=0$. In Umfangsrichtung sind periodische Lösungen der Form $\sim \exp (i m \phi)$ möglich. Für ein gegebenes $m$ setzt sich die Lösung aus Besselfunktionen $J_{m}$ und Neumannfunktionen $Y_{m}$ (auch Besselfunktion 2. Gattung genannt) zusammen: 


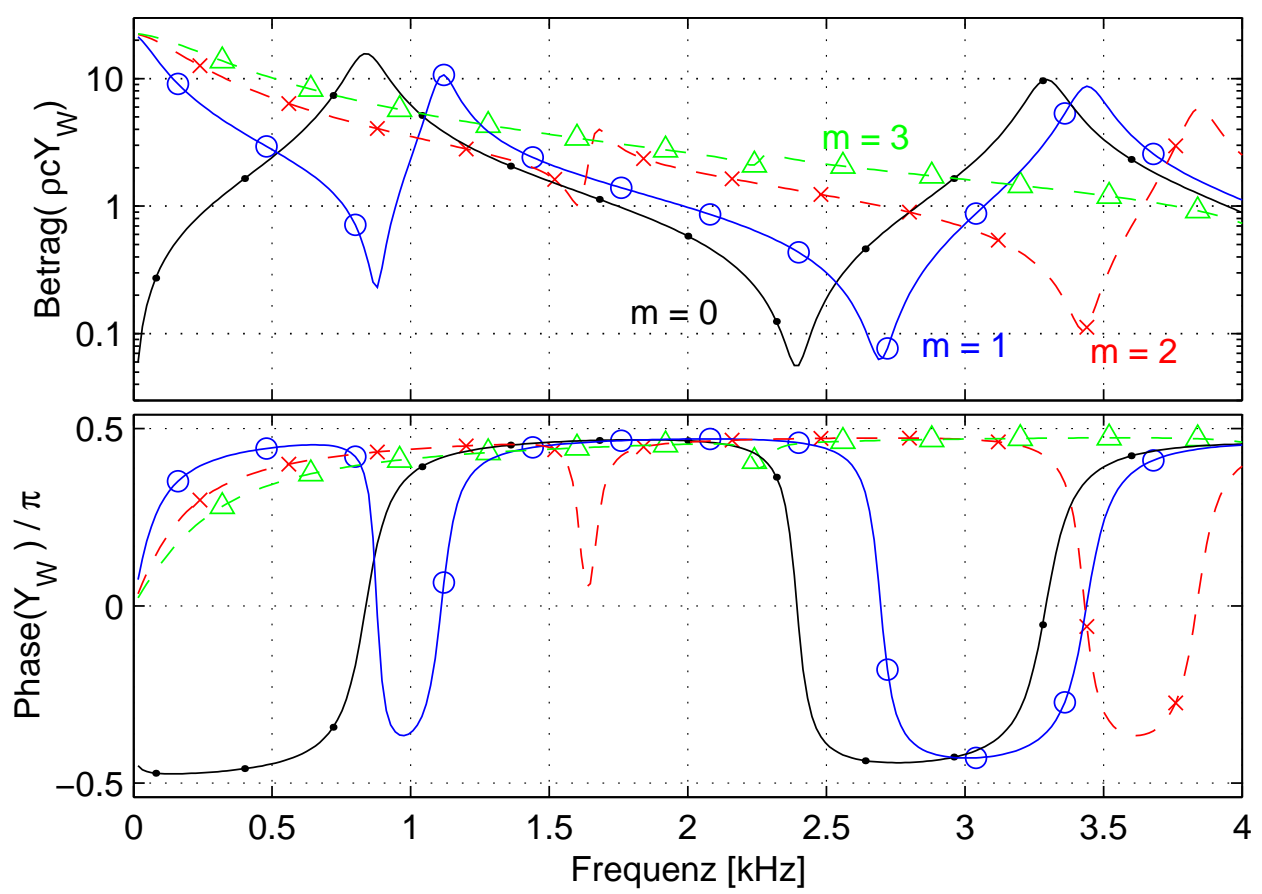

Abb. 2.4: Wandadmittanz im Resonatorabschnitt für einige zirkulare Moden, $p \sim \exp (i m \phi)$ nach Gleichung 2.4 .

$$
\begin{aligned}
\rho c Y_{\mathrm{W}}^{\prime} & =i \frac{m}{k_{r, \mathrm{~W}} R}-i \frac{J_{m-1}\left(k_{r, \mathrm{~W}} R\right)+a_{m} Y_{m-1}\left(k_{r, \mathrm{~W}} R\right)}{J_{m}\left(k_{r, \mathrm{~W}} R\right)+a_{m} Y_{m}\left(k_{r, \mathrm{~W}} R\right)} \quad \mathrm{mit} \\
a_{m} & =-\frac{k_{r, \mathrm{~W}} R_{a} J_{m-1}\left(k_{r, \mathrm{~W}} R_{a}\right)-m J_{m}\left(k_{r, \mathrm{~W}} R_{a}\right)}{k_{r, \mathrm{~W}} R_{a} Y_{m-1}\left(k_{r, \mathrm{~W}} R_{a}\right)-m Y_{m}\left(k_{r, \mathrm{~W}} R_{a}\right)}
\end{aligned}
$$

Die Schallgeschwindigkeit ist mit $c$, die Dichte mit $\rho$, und die Wellenzahl in radialer Richtung mit $k_{r, \mathrm{~W}}$ bezeichnet. In den Resonatorkammern gilt - bis auf eine Dämpfung $k_{r, \mathrm{~W}}=\frac{\omega}{c}$. Die Dämpfung wird in der Wellenzahl berücksichtigt, die Verluste durch viskose Reibung und Wärmeleitung an der Wand der Resonatorkammer einbezieht und über die Kammerbreite mittelt (siehe z. B. Cremer [6]). Damit ergibt sich für die über eine Kammer gemittelte Wellenzahl ${ }^{1}$

$$
k_{r, \mathrm{~W}}=\frac{\omega}{c}\left[1+\frac{\delta}{B}(1+i)\left(1+\frac{\gamma-1}{\mathrm{Pr}}\right)\right]
$$

\footnotetext{
${ }^{1}$ In dieser Arbeit wird die Konvention verwendet, dass akustische Wechselgrößen sich $\sim \exp (-i \omega t)$ verhalten. Von dem Vorzeichen des Exponenten hängt das Vorzeichen des Imaginärteils der Wellenzahl ab.
} 
mit der Stokesdicke $\delta=\sqrt{2 \nu / \omega}$, der kinematischen Zähigkeit $\nu$ und der Prandtlzahl $\operatorname{Pr}=0,713$.

Ein Drahtgitter trennt die Resonatorkammern von dem durchströmten Rohr. Akustisch äußert sich dies in einer zusätzlichen Impedanz, die von Förster [9] frequenzun-

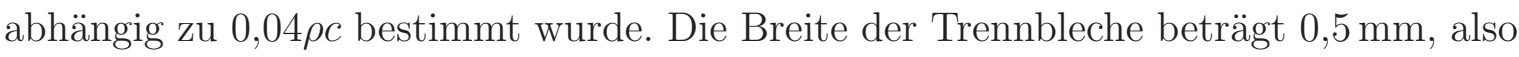
$10 \%$ der Kammerbreite. An der Kanalseite dieser Trennblechen ist $u_{r}=0$. Werden diese beiden Korrekturen noch in der Wandadmittanz berücksichtigt ergibt sich

$$
Y_{\mathrm{W}}=\frac{Y_{\mathrm{W}}^{\prime}}{1,1 \cdot\left(1+0,04 \rho c Y_{\mathrm{W}}^{\prime}\right)}
$$

Abb. 2.4 zeigt die Wandadmittanz als Funktion der Frequenz für die radialsymmetrische Mode $m=0$ und die höheren Moden bis $m=3$. Die genaue Lage der Resonanzen hängt von der Schallgeschwindigkeit und der Dämpfung ab. Hier ist $c=343 \mathrm{~m} / \mathrm{s}$ gesetzt.

Die ersten beiden Resonanzen im Fall $m=0$ liegen mit den angegebenen Parametern bei $840 \mathrm{~Hz}$ und $3290 \mathrm{~Hz}$, die Antiresonanz bei $2390 \mathrm{~Hz}$. Für $m=1$ sind die Resonanzen bei $1110 \mathrm{~Hz}$ und $3440 \mathrm{~Hz}$ sowie die Antiresonanzen bei $880 \mathrm{~Hz}$ und $2700 \mathrm{~Hz}$.

\subsection{Verhalten bei Strömung}

In diesem Kapitel wird der Einfluss der Wandauskleidungen auf das akustische Spektrum im Kanal bei Strömungsüberlagerung betrachtet. Zunächst sind die Messungen für das nicht beschallte Rohr gezeigt. Anschließend wird der Einfluss von zusätzlich eingestrahltem Schall diskutiert. Das Hauptinteresse liegt auf der Messung der Druckschwankungen im Rohr stromab der Auskleidung.

\subsubsection{Spektren der Druckschwankungen}

Abb. 2.5 zeigt die spektrale Leistungsdichte der turbulenten Druckschwankungen im Rohr stromab des Resonatorabschnitts in willkürlichen Einheiten (w. E.) für unterschiedliche Machzahlen. Definiert ist die Machzahl in dieser Arbeit als auf die Schallgeschwindigkeit normierte und über den Rohrquerschnitt gemittelte Strömungsgeschwindigkeit, $\mathrm{Ma}=\bar{u} / c$. Die Spektren sind durch den runden Resonatorabschnitt mit 16 


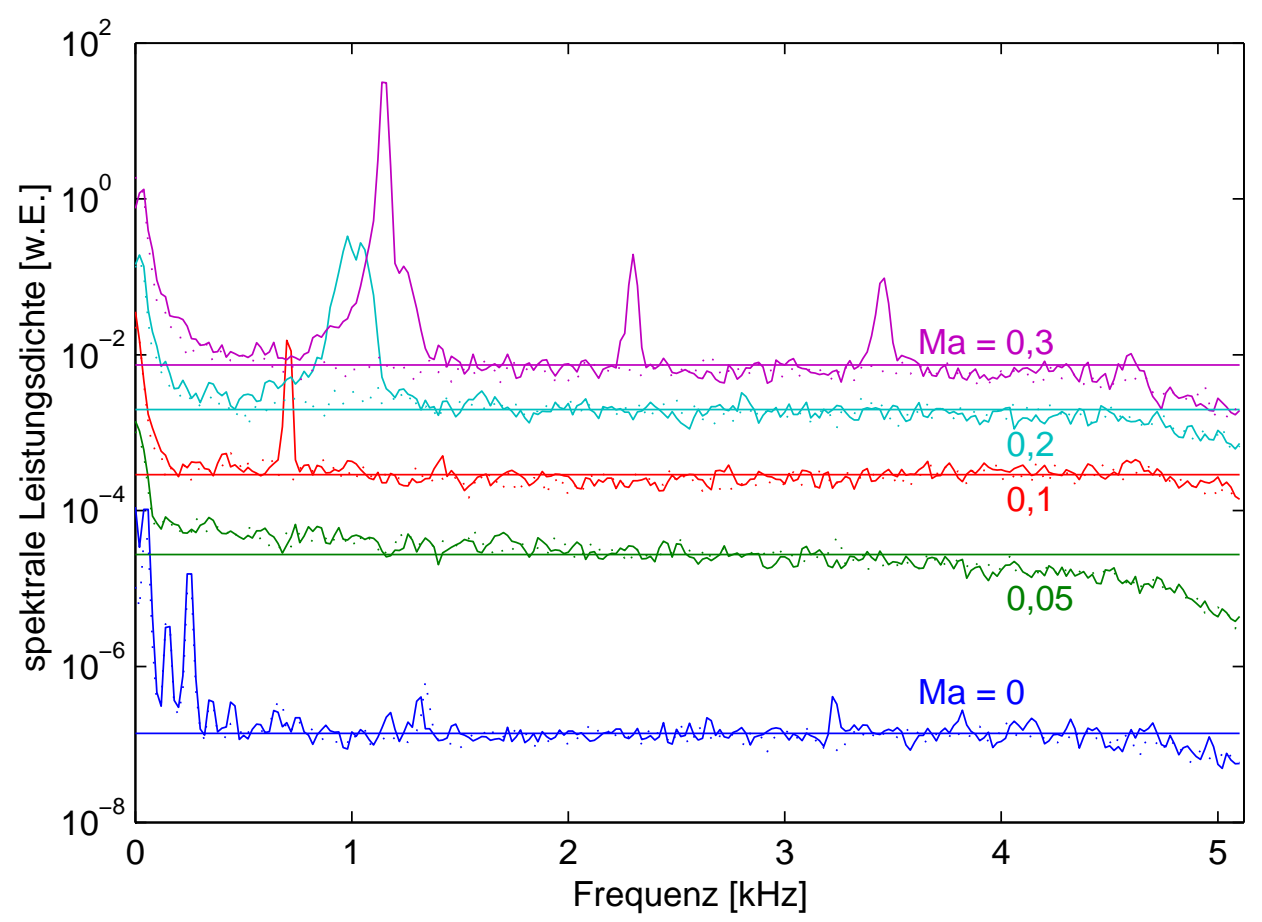

Abb. 2.5: Spektrale Leistungsdichte stromab des Resonatorabschnitts mit 16 Resonatoren (durchgezogene Kurven), deren Median-Werte (waagerechte Linien) sowie Vergleichsmessungen ohne Resonatorabschnitt (gepunktet).

Kammern spektral verfärbt; zum Vergleich sind die Kurven aus dem Rohr ohne Einbauten gepunktet eingezeichnet. Die Samplingfrequenz beträgt 10,24kHz. Die Spektren sind aus Zeitreihen mit 8192 Samples gewonnen, die in 512 Samples lange Abschnitte zerlegt mit einem Hanning-Fenster gemittelt wurden. Die Frequenzauflösung beträgt somit $40 \mathrm{~Hz}$.

Oberhalb von $4,5 \mathrm{kHz}$ beginnt ein Tiefpass die spektrale Leistungsdichte abzuschneiden. Bei tiefen Frequenzen bis ca. $300 \mathrm{~Hz}$ treten sehr große Druckschwankungen auf; darüber ist das Spektrum für Machzahlen ab 0,05 - bis auf Einflüsse durch die Resonatoren - praktisch unabhängig von der Frequenz.

Zusätzlich sind Medianwerte der spektralen Leistungsdichte eingezeichnet. Für die weitere Auswertung (siehe Abb. 2.6) wird die spektrale Leistungsdichte auf den jeweiligen Median bezogen und farbcodiert aufgetragen. Aufgelöst wird der Pegelbereich zwischen $0 \mathrm{~dB}$ und $40 \mathrm{~dB}$, kleinere Werte sind weiß, größere schwarz gezeichnet. Der Farbverlauf ist in der Farbtabelle $0.1 \mathrm{im}$ Symbolverzeichnis zu sehen. Im Verlauf mit 


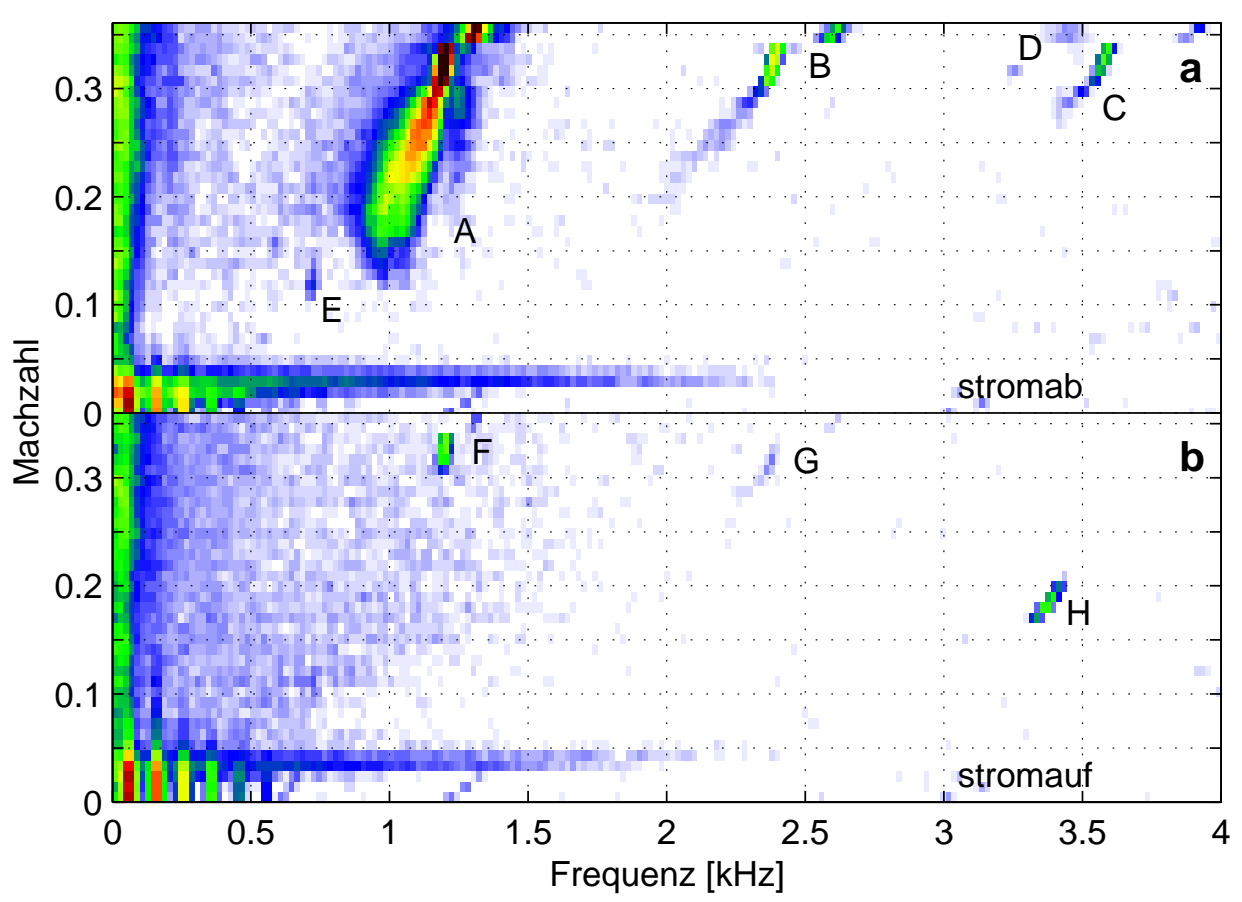

Abb. 2.6: Druckspektren mit 16 Resonatoren für Machzahlen bis 0,355 gemessen im Rohr stromab (oben) und stromauf (unten) des Resonatorabschnitts; die farbcodierte Leistungsdichte ist auf den jeweiligen Median-Wert bezogen. Siehe Farbtabelle 0.2 im Symbolverzeichnis.

der Machzahl lassen sich spektrale Überhöhungen so gut erkennen². Da der Median der Referenzwert ist, ist mindestens die Hälfte jeder Farbzeile weiß3. Für Ma $\lesssim 0,05$ ist der bereits erwähnte Pegelanstieg zu tiefen Frequenzen hin zu sehen. Bei noch kleineren Machzahlen kommt bei $50 \mathrm{~Hz}$ und deren ungeraden Harmonischen zunehmend das Übersprechen der Versorgungsspannung in den Messgeräten heraus.

Abb. 2.6 zeigt die Spektren in der beschriebenen Codierung in Abhängigkeit von der Machzahl für zwei Mikrofonpositionen. In der oben gezeigten Messung befindet sich das Mikrofon stromab, in der unteren ist es stromauf des Resonatorabschnitts angebracht. Die Abstände sind groß im Vergleich zum Rohrdurchmesser.

Mehrere Bereiche spektraler Überhöhungen sind hier zu sehen. Besonderes Interesse

${ }^{2}$ Statt des Medians könnte hier auch das Spektrum im leeren Rohr abgezogen werden. Jedoch wäre dann bei Messungen mit anderem Kanalquerschnitt nicht klar, welchen Wert die ReferenzMachzahl hat. Zudem wird zur Bestimmung des Medians keine Referenzmessung benötigt.

3 Tatsächlich wird erst der Bereich ab 0,6 dB oberhalb des Medians farbcodiert, wenn viele Werte sehr dicht am Median liegen, ist deutlich mehr als eine halbe Farbzeile weiß. 
gilt dem mit A gekennzeichneten Bereich bei Frequenzen etwas oberhalb der Kammerresonanz von $840 \mathrm{~Hz}$. Ab Ma $=0,12$ ist er um $980 \mathrm{~Hz}$ zu erkennen. Mit wachsender Machzahl wird die Überhöhung deutlich stärker, das Maximum verschiebt sich zu höheren Frequenzen und erreicht $1,2 \mathrm{kHz}$ bei Ma $=0,34$, wobei der Frequenzbereich immer schmaler wird. Bei der nächsthöheren Machzahl 0,35 springt die Frequenz des Pegelmaximums auf $1,3 \mathrm{kHz}$ und verschiebt sich mit der Machzahl weiter zu höheren Frequenzen; maximal wird $\mathrm{Ma}=0,355$ erreicht. Bei Strömungen ab $\mathrm{Ma}=0,2$, bzw. deutlich ab 0,29 treten höhere Harmonische dieser Instabilitätsfrequenz auf (mit B und C gekennzeichnet). Ihre größten Amplituden erreichen sie vor dem Sprung über die Grenze bei 1,2 kHz. Hier strahlt die Grundfrequenz und die erste Harmonische auch in das Rohr stromauf (Abb. 2.6 unten, Bereiche F und G). Das deutet darauf hin, dass sich aus der konvektiven Instabilität eine selbsterregte Schwingung (oder globale Instabilität) entwickelt 4 .

Schwach zu sehen ist für große Machzahlen bei etwa $3,4 \mathrm{kHz}$ eine Überhöhung (D), wieder etwas oberhalb der Frequenz, bei der die 2. Kammerresonanz liegt, vgl. Abb. 2.4.

Stromab tritt ein Bereich bei $710 \mathrm{~Hz}$ zwischen Ma = 0,11 und 0,14 leicht hervor (E). Eine weitere Überhöhung ist stromauf um $3,4 \mathrm{kHz}$ bei Ma von 0,17 bis $0,20 \mathrm{zu}$ sehen (H). Diese beiden Überhöhungen werden hier nicht näher betrachtet.

\subsubsection{Einfluss der Resonatorenzahl}

Besondere Beachtung soll dem zuerst erwähnten Verstärkungsbereich A zuteil werden. In Abb. 2.7 sind oben die erreichten maximalen Erhöhungen über dem Medianwert in Abhängigkeit von der Machzahl aufgetragen. Ähnliche Messungen wurden mit unterschiedlicher Anzahl von offenen Resonatorkammern wiederholt - also für verschiedene Längen der Wandauskleidung. Das eigentliche Messobjekt besteht aus 16 Resonatoren; die Messungen mit weniger Resonatoren werden mit abgeklebten stromaufseitigen Resonatoren durchgeführt. Zur Auswertung der Frequenzabhängigkeit werden 128 Samples lange Abschnitte fouriertransformiert und auf $5 \mathrm{~Hz}$ interpoliert. Besonders für niedrige Machzahlen, also kleinen Überhöhungen, werden wegen der breiteren Mittelung bessere Ergebnisse erzielt.

\footnotetext{
${ }^{4}$ Da die physikalischen Prinzipien der Instabilität nicht klar sind, kann streng genommen nicht entschieden werden, ob es sich um eine globale Instabilität oder um eine absolute Instabilität handelt. Im Folgenden wird sie als globale Instabilität bezeichnet, was insofern als schwächere Formulierung betrachtet werden kann, als eine absolute Instabilität im Wellenzahlraum gefunden wird und somit ebenfalls eine globale Betrachtung voraussetzt [11].
} 

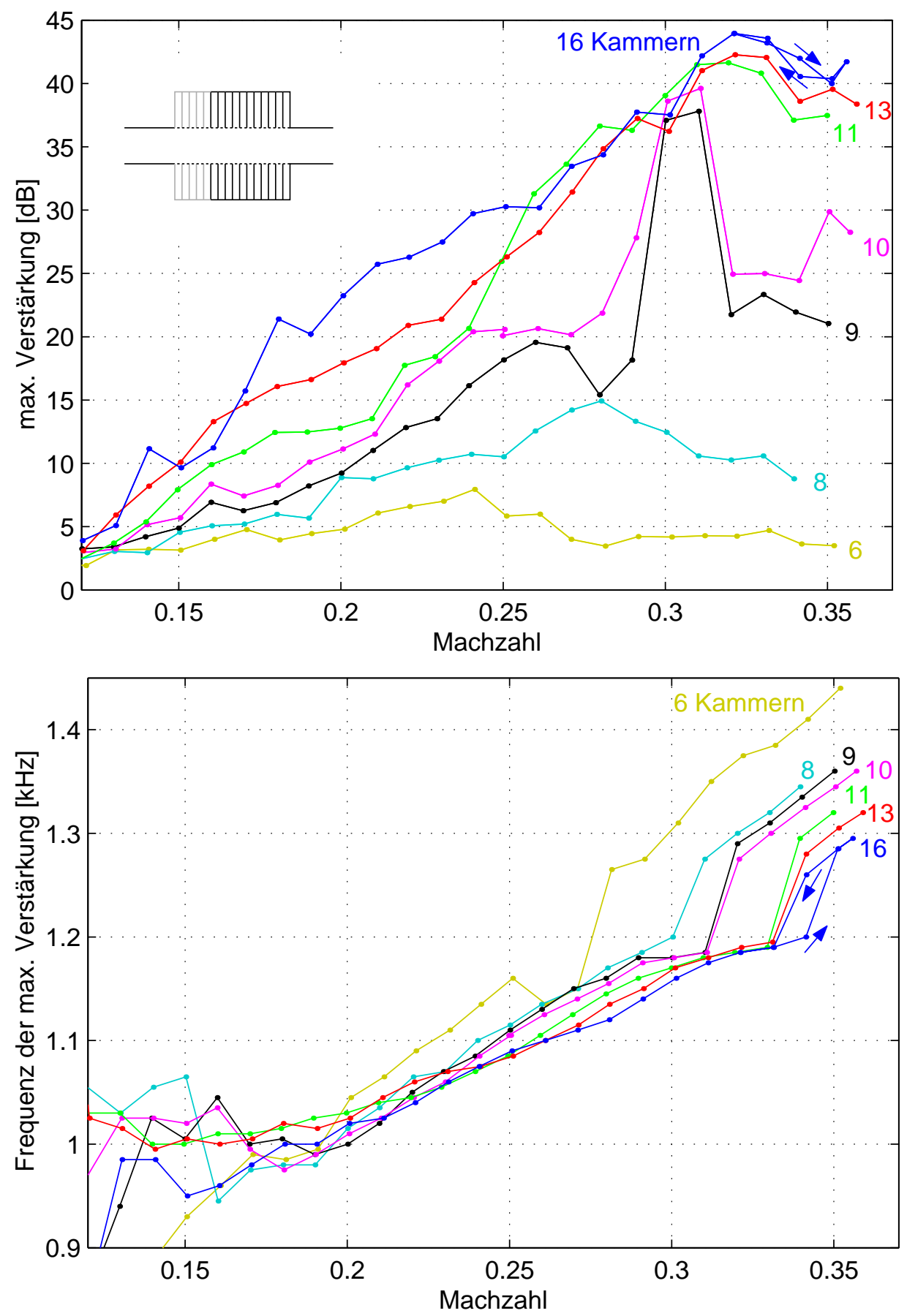

Abb. 2.7: Maximale spektrale Drucküberhöhung zwischen 0,85 und $2 \mathrm{kHz}$ (oben) und zugehörige Frequenz $f_{\max }$ (unten) stromab des Resonatorabschnitts. Parameter ist die Anzahl offener Resonatorkammern. Um Ma $=0,34$ tritt Hysterese auf, die Umlaufrichtung ist durch Pfeile angezeigt 
Die Verstärkung nimmt mit wachsender Resonatorenzahl zu. Das erscheint zunächst naheliegend, allerdings hatten Messungen von Brandes an einem Resonatorabschnitt mit einer Resonanzfrequenz von $2960 \mathrm{~Hz}$ und mit acht bzw. mit 16 Kammern an dem kürzeren Abschnitt einen etwas größeren Transmissionsfaktor aufgewiesen [2, Abb. 4.5].

Beachtlich ist hier der Unterschied zwischen acht und neun Resonatorkammern. Bei acht Kammern ist noch kein Ansatz einer Aufsteilung der Amplitude zu erkennen, wenn sich die Frequenz, bei der die maximale Verstärkung auftritt, 1,2 kHz nähert. Mit neun Kammern wird in der Aufsteilung ein Wert erreicht, der sich bis zu 16 Kammern nicht mehr wesentlich erhöht.

Klar zu sehen ist für alle Resonatorenzahlen der Anstieg der am stärksten verstärkten Frequenz $f_{\max }$ mit der Machzahl. Bemerkenswert ist, dass sich die Frequenzen $f_{\max }$ oberhalb von $\mathrm{Ma}=0,23$ mit steigender Machzahl zunehmend langsamer an 1,20 kHz nähern, und dann zu Frequenzen oberhalb von 1,26 kHz springen, ohne Zwischenwerte anzunehmen. Bevor diese Schwelle bei $1,20 \mathrm{kHz}$ übersprungen wird, steilt sich die Amplitude beträchtlich auf - besonders deutlich ist das in Abb. 2.7 bei neun und zehn Resonatoren zu sehen. Damit wird der Frequenzbereich zunehmend schmaler bis schließlich ein nahezu monofrequenter Ton vorliegt. Dabei wird der Wert der maximalen Verstärkung wenig aussagekräftig, da immer über einen $40 \mathrm{~Hz}$ breiten Bereich gemittelt wird. Oberhalb von $1,26 \mathrm{kHz}$ ist die Amplitude wieder geringer, der Frequenzbereich verbreitet sich ein wenig.

Bei den Messungen wurde die Machzahl kontinuierlich erhöht. Nur für den Fall mit 16 Kammern ist hier zusätzlich das Verhalten eingezeichnet, wenn die Machzahl wieder verringert wird. Die Pfeile zeigen die Richtung der Änderung an. Um Ma =0,34 liegt Hysterese vor, die ein Zeichen für eine globale Instabilität ist.

\subsubsection{Einfluss der Querschnittsgeometrie}

Mit einem achsensymmetrischen Zentralkörper in der Kanalmitte (s. Abb. 2.3) ändert sich die Verstärkung und die zugehörige Frequenz $f_{\max }$, wie in Abb. 2.8 gezeigt. Als Parameter ist die Breite des für die Strömung frei bleibenden ringförmigen Spaltes $S$ angegeben. Der Durchmesser des Zentralkörpers nimmt kurz vor den Resonatoren stetig zu und erreicht etwa bei der ersten Kammer seinen Maximalwert. Der Zentralkörper lässt einen ringförmigen Querschnitt frei, dessen Breite jeweils angegeben ist. Die Machzahl bezieht sich auf die Strömung in diesem Spalt. Stromab nimmt der Durchmesser des Zentralkörpers wieder stetig ab. In den Teilrohren stromauf und 

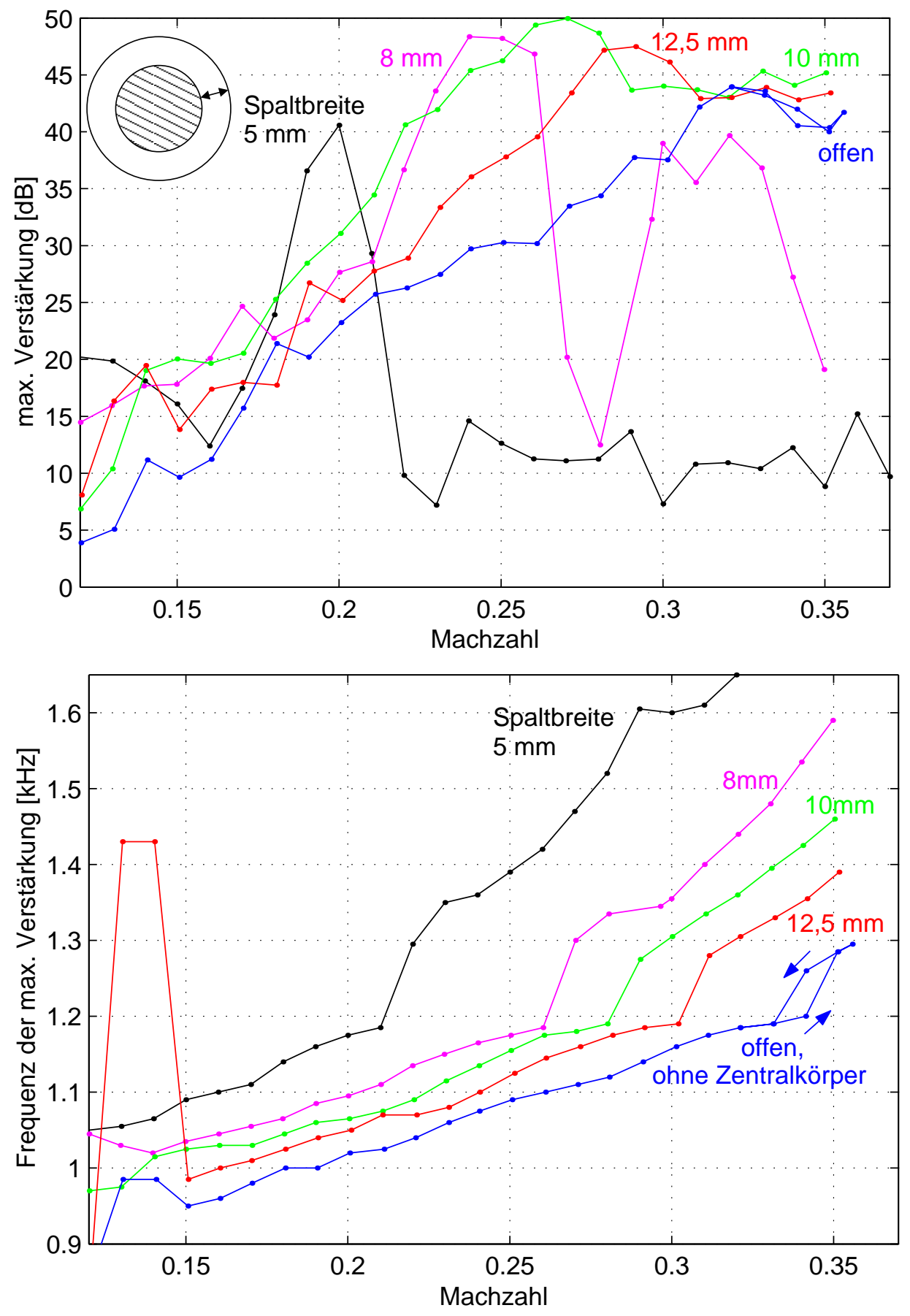

Abb. 2.8: Wie Abb. 2.7, jedoch für 16 Resonatorkammern mit verschiedenen Zentralkörpern, also unterschiedlichen Breiten der freien Ringspalte 
stromab ist die Strömungsgeschwindigkeit also bis auf kompressible Effekte um das Querschnittsverhältnis zwischen Ringspalt und Messrohr kleiner.

Auch hier steigt die Frequenz $f_{\max }$ mit der Strömungsgeschwindigkeit an. Frequenzen zwischen 1,20 und 1,26 kHz werden auch hier nicht angenommen. Je schmaler der Spalt wird, desto größer werden die Frequenzen. Dabei bleibt die Steigung mit der Machzahl etwa konstant. Je weiter $f_{\max }$ mit steigender Machzahl in die Nähe von 1,2 kHz kommt, desto größer wird die Verstärkung. Nach dem Sprung von $f_{\max }$ auf Frequenzen oberhalb $1,26 \mathrm{kHz}$ verringert sich die zugehörige Verstärkung etwas, ähnlich wie es bei den Kurven in Abb. 2.7 zu sehen ist. Die maximale Verstärkung wird bei einer Spaltbreite von $10 \mathrm{~mm}$ erreicht. Bei kleineren Spalten nimmt die Verstärkung deutlicher ab, wenn Machzahlen erreicht werden, bei denen $f_{\max }$ oberhalb $1,26 \mathrm{kHz}$ liegt.

\subsubsection{Amplitudenstatistik der Druckschwankungen}

Nun soll die Statistik der Mikrofonsignale untersucht werden. Turbulente Druck- und Geschwindigkeitsschwankungen laufen mit der Strömung in den Resonatorabschnitt. Wenn eine konvektive Instabilität vorliegt, werden sie - abhängig von ihrer Frequenz verstärkt oder gedämpft. Die Amplituden der Signale im Strömungsrauschen sind gaußverteilt; sie bleiben es auch bei einer linearen Verstärkung. Führt eine Nichtlinearität zu einer Begrenzung der Amplitude, beispielsweise in einer globalen Instabilität, so ändert sich die Amplitudenverteilung. Im einfachsten Fall entsteht eine Sinusschwingung. Die Signalamplituden sind dann nicht mehr gaußverteilt.

Abb. 2.9 zeigt einen Vergleich der Verteilung des Betrags der gesampelten Mikrofonwerte $w_{i}$ mit einer Normalverteilung und der Verteilungsfunktion eines Sinussignals. Gezeigt sind Kurven für 16 offene Resonatorkammern bei freiem Querschnitt. Die Samples sind auf ihren RMS-Wert $\sigma$ normiert. Nun wird die Anzahl der Werte ermittelt, die in Abschnitten (engl. Bins) mit $n / 5<\left|w_{i} / \sigma\right|<(n+1) / 5$ liegen, wobei

$n \in\{0,1,2, \ldots\}$. Diese Anzahl wird über $\left(\frac{2 n+1}{10}\right)^{2}$ halblogarithmisch aufgetragen. Eine Normalverteilung ergibt somit eine Gerade. Neben dieser Geraden ist mit einer gestrichelten Linie die Amplitudenverteilung einer Sinusfunktion eingetragen, die in Bins der gleichen Größe eingeteilt ist. Mit steigender Machzahl ähneln die Kurven zunehmend der Sinusverteilung, nur bei $\mathrm{Ma}=0,35$, also wenn $f_{\max }$ über $1,2 \mathrm{kHz}$ springt, ändert sie sich leicht in Richtung einer Gaußverteilung.

Als Maß für die Abweichung von einer Normalverteilung ist in der kleinen Grafik für die gesampelten Werte zu allen Machzahlen der Wert des $\chi^{2}$-Tests aufgetragen (siehe z. B. [4]). Man sieht, dass ab $\mathrm{Ma}=0,22$ die Abweichung von der Normalverteilung zunimmt und ab $\mathrm{Ma}=0,29$ sprunghaft ansteigt. Das deckt sich mit dem Verlauf 


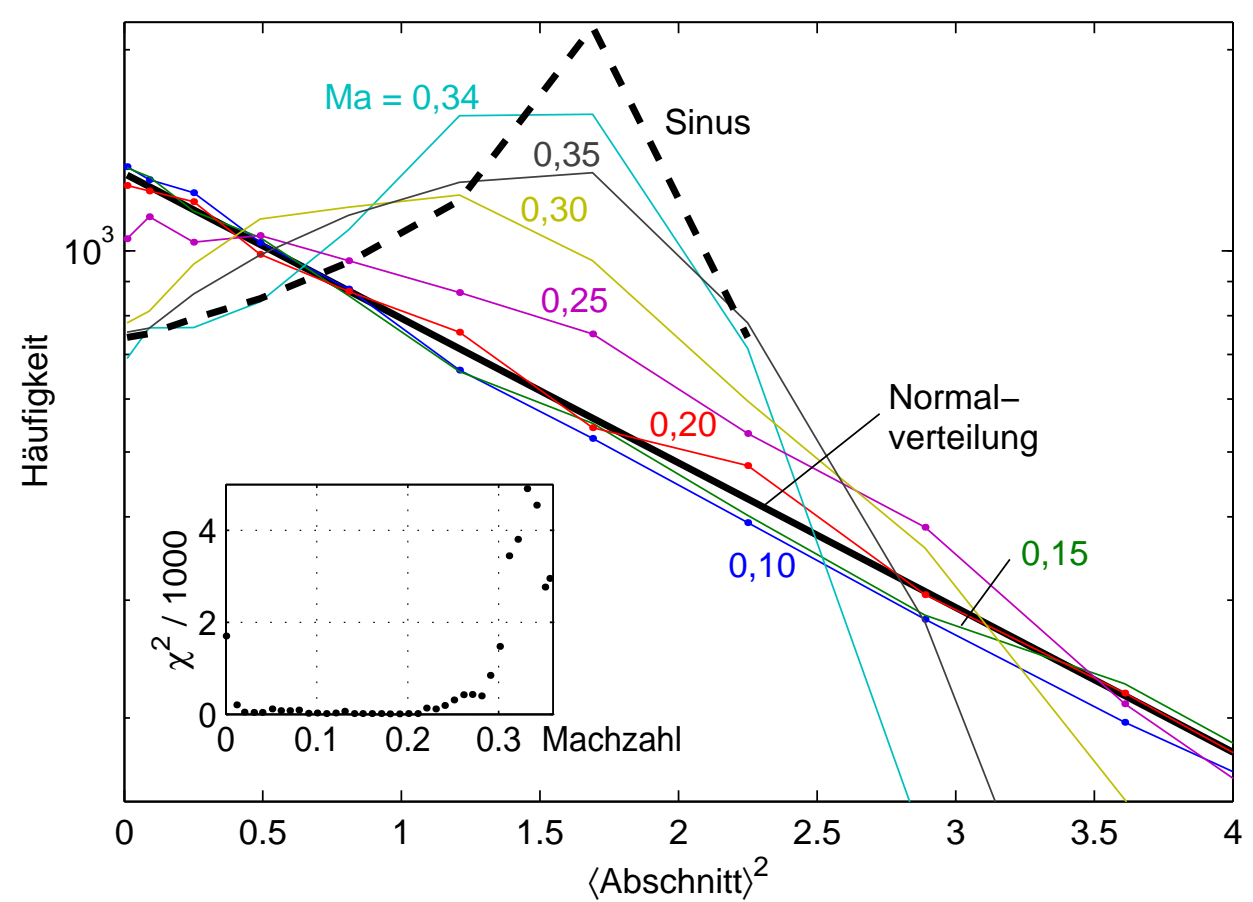

Abb. 2.9: Amplitudenverteilung der quadrierten, auf die Varianz bezogenen Mikrofonsignale (Bin-Breite 0,2) für ausgewählte Machzahlen, im Vergleich mit den Verteilungen eines normalverteilten und eines Sinussignals. Die kleine Auftragung zeigt einen $\chi^{2}$-Vergleich zwischen den Verteilungen des Mikrofonsignals und eines normalverteilten Signals als Funktion der Machzahl.

des Spektrums aus Abb. 2.6, in dem bei dieser Machzahl die doppelte Grundfrequenz $2 f_{\max }$ zunehmend hervortritt.

Die hier analysierten Werte stammen aus der Frequenzanalyse und sind daher nicht statistisch unabhängig voneinander. Die ermittelten $\chi^{2}$-Werte können daher nur zu qualitativen Aussagen herangezogen werden. Aussagen zur Signifikanz der Abweichung von der Normalverteilung sind nicht möglich.

\subsection{Druckamplituden bei Beschallung}

\subsubsection{Die Amplitudenabhängigkeit der Streufaktoren}

In diesem Abschnitt wird zunächst die Abhängigkeit der Schalltransmission und -reflexion von der Amplitude stromauf eingebrachten, monofrequenten Schalls be- 


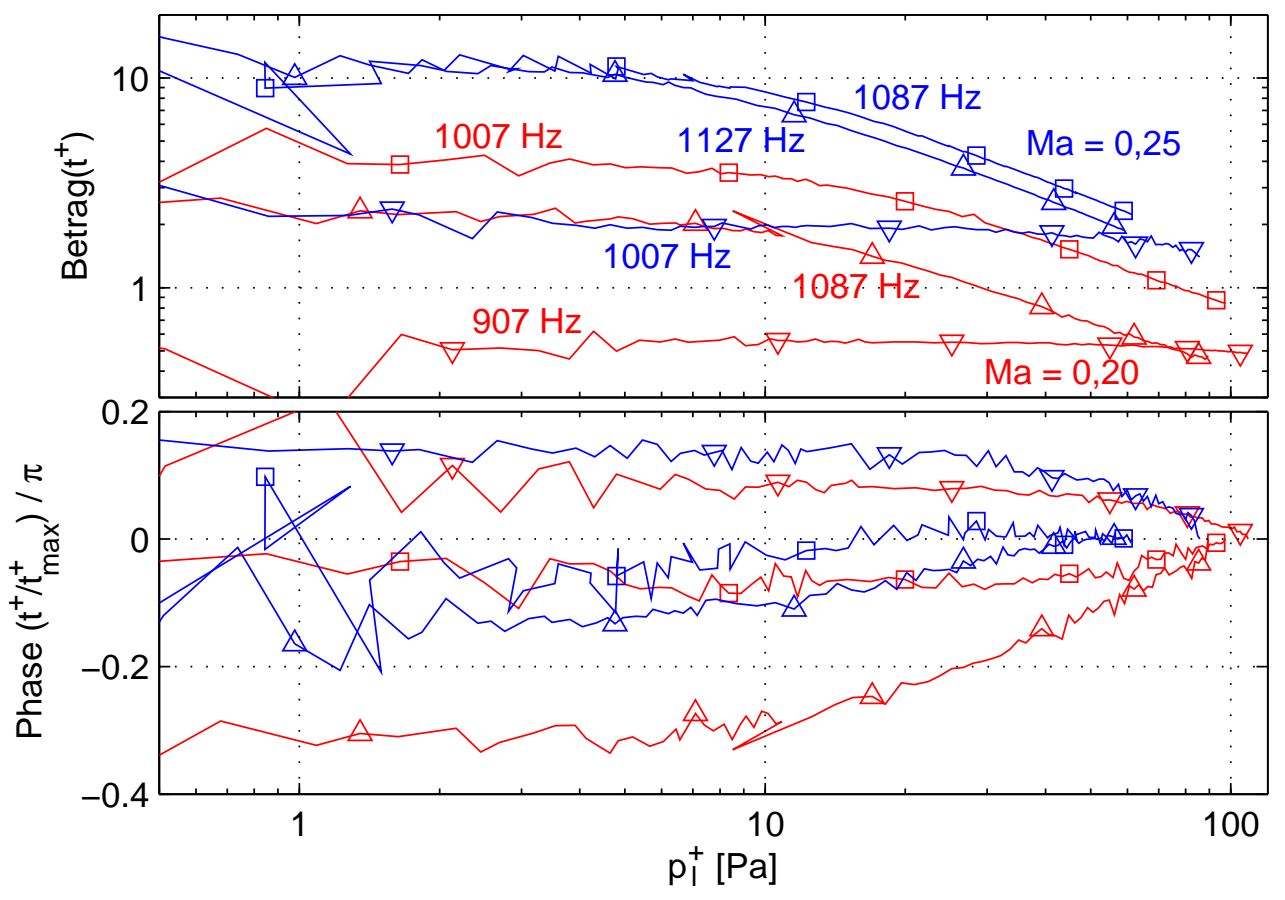

Abb. 2.10: Betrag und Phase des Transmissionsfaktors in Strömungsrichtung $t^{+}$als Funktion der Amplitude der einlaufenden Welle für $\mathrm{Ma}=0,2(\operatorname{rot}, \nabla: 907 \mathrm{~Hz}, \square: 1007 \mathrm{~Hz}, \triangle: 1087 \mathrm{~Hz})$, für $\mathrm{Ma}=0,25$ (blau, $\nabla: 1007 \mathrm{~Hz}, \square: 1087 \mathrm{~Hz}, \triangle: 1127 \mathrm{~Hz}$ ).

trachtet. Anschließend wird der Einfluss des Schalls auf das turbulente, durch den Resonatorabschnitt verfärbte Spektrum im Rohr gezeigt.

An zwei verschiedenen Stellen kann Schall eingebracht werden: Eine Lautsprecherposition befindet sich im Rohr stromauf. Als zweite Möglichkeit können an der ersten Resonatorkammer drei Lautsprecher je $120^{\circ}$ in Umfangsrichtung versetzt angebracht werden.

\section{Beschallung aus dem Rohr stromauf}

Um die Linearität des Resonatorabschnitts bezüglich der eingestrahlten Schallamplitude zu beurteilen, wird für verschiedene Machzahlen und Frequenzen die Abhängigkeit des Stehwellenfeldes in den Messrohren von der Beschallungsamplitude $A_{s}$ gemessen. Die Mikrofonsignale werden phasenstarr zur anregenden Frequenz über fünf Sekunden gemittelt. An die Messwerte der Mikrofone (die zuvor ohne Strömung und ohne Resonatorabschnitt geeicht werden) wird in jedem Teilrohr eine nach rechts und eine nach 
links laufende Schallwelle angepasst. Das Verhältnis der im linken Teilrohr an Resonatoren reflektierten Schallamplitude ${ }^{5} p_{l}^{-}$zu der aus Strömungsrichtung einlaufenden $p_{l}^{+}$ist der Reflexionsfaktor $r^{+}:=p_{l}^{-} / p_{l}^{+}$. Das Verhältnis der durch die Resonatoren transmittierten Schallwelle $p_{r}^{+}$zu $p_{l}^{+}$ist der Transmissionsfaktor $t^{+}:=p_{r}^{+} / p_{l}^{+}$. Da hier nur aus dem linken Teilrohr beschallt wird, muss zudem angenommen werden, dass kein stromauf laufender Schall aus dem rechten Teilrohr ins linke gelangt, dass also das Produkt aus dem Reflexionsfaktor am stromabseitigen Rohrende und dem Transmissionsfaktor im Resonatorabschnitt gegen die Strömungsrichtung sehr klein ist. Für die betrachteten Frequenzen liegt der Wert nach Brandes [2] und Lange [15] in der Größenordnung von 0,001.

Abb. 2.10 zeigt Betrag und Phase des Transmissionsfaktors $t^{+}$als Funktion von $p_{l}^{+}$ für $\mathrm{Ma}=0,25$ (blaue Kurve) bei Frequenzen oberhalb (mit dem Symbol $\triangle$ markiert), etwa gleich $(\square)$, sowie unterhalb $(\nabla)$ der Frequenz maximaler Verstärkung $f_{\max }$. Mit gleicher Bedeutung der Symbole sind für $\mathrm{Ma}=0,2$ (rot) drei Kurven eingetragen. Die Werte sind gegen die Amplitude des Schalls in der Einheit Pascal aufgetragen. Diese Angabe des absoluten Schalldrucks soll die Größenordnung verdeutlichen, eine kalibrierte Bestimmung war nicht möglich ${ }^{6}$. Die gemessene Anregungsamplitude wird mit $p_{l}^{+}$bezeichnet, wenn diese nicht ermittelt wurde, wird die Amplitude $A_{s}$ verwendet, die der Lautsprecheransteuerung dient. Beide Größen sind proportional, die Proportionalitätskonstante hängt jedoch von der Frequenz und der Strömungsgeschwindigkeit ab. Bei den hier gezeigten verschiedenen Frequenzen und Machzahlen schwankt die Maximalamplitude, die mit dem Lautsprecher erreicht wird, um den Faktor 2, was u. a. an der sich ändernden Impedanz an der Lautsprecherposition im Messrohr liegt, wie auch an der Frequenzabhängigkeit des Lautsprechers selbst, siehe Abb. A.1.

Dem Transmissionsfaktor im Grenzfall kleiner Amplituden kommt eine besondere Bedeutung zu. In diesem Fall hängt die Amplitude der transmittierten Schallwelle linear von der Amplitude der einlaufenden Welle ab. Die Gleichströmung bleibt vom Schall unbeeinflusst. Im durchströmten Rohr wird mit kleinen Amplituden das Signal-zuRausch-Verhältnis ungünstig, die zugehörigen Messkurven werden verrauschter.

Die Frequenzen liegen alle im Bereich der Instabilität. Der Betrag von $t^{+}$nimmt mit wachsendem Schalldruck ab, nur die Kurve für die jeweils niedrigste Frequenz bleibt nahezu konstant. Die Transmissionsfaktoren zu $f_{\max }$ und zu der nächsthöheren Fre-

\footnotetext{
${ }^{5}$ Der obere Index gibt die Laufrichtung in $(+)$ bzw. gegen $(-)$ die Strömung an, der untere Index das Teilrohr, stromauf oder links $(l)$ bzw. stromab oder rechts $(r)$.

${ }^{6}$ Es sind Ablagerungen auf dem Mikrofonmembran zu sehen; der Raum vor dem Mikrofon bildet mit der Bohrung einen Resonator mit einer Resonanz bei ca. $10 \mathrm{kHz}$ [1, S. 34]; die Messgerätekette ist seit langem nicht kalibriert worden.
} 
quenz nehmen von den kleinsten zu den größten Amplituden etwa um den Faktor 5 ab.

Es scheint, dass sich $t^{+}\left(p_{l}^{+}\right)$in zwei Bereiche einteilen lässt: Für kleine Amplituden ist $t^{+}$konstant, für große $p_{l}^{+}$ist $t^{+} \sim\left(p_{l}^{+}\right)^{-1+\epsilon}$, mit $\epsilon \geq 0$, abhängig von der Machzahl und Frequenz. Der Übergang erfolgt allmählich bei jeweils unterschiedlichen Amplituden, hier mit $\mathrm{Ma}=0,25$ für beide höheren Frequenzen etwa bei $8 \mathrm{~Pa}$ und mit $\mathrm{Ma}=0,20$ für die höheren Frequenzen bei ca. 15 Pa, bevor die Sättigung einsetzt.

Möglicherweise tritt die Sättigung durch eine Änderung des mittleren Strömungsprofils ein, das sich mit ansteigender Schallamplitude zunehmend verformt. Für kleine Amplituden ist die Verformung gering, die transmittierte Welle $p_{r}^{+}$hängt dann linear von der Amplitude der einlaufenden Welle ab. Bei großen Amplituden dagegen tritt eine Sättigung im Lauf durch den Resonatorabschnitt auf, das Profil ist so weit verformt, dass eine weitere Verstärkung nicht stattfinden kann. Es kommt zu einem Gleichgewicht zwischen der Verformung durch die verstärkte Schallwelle und dem Druckgradienten bzw. der zeitlich gemittelten Schubspannung, so dass $p_{r}^{+}$unabhängig von der einlaufenden Welle wird. Bei immer weiter steigender Amplitude (die hier nicht mehr erreicht wird), sollte schließlich eine andere akustische Mode für die Transmission an Bedeutung gewinnen und $t^{+}$wieder für einen Amplitudenbereich konstant sein, bevor die Nichtlinearität durch die Kompression der Luft eintritt.

In Abb. 2.10 (unten) ist die Phase von $t^{+}$bei der Maximalamplitude auf Null gedreht. So lässt sich ihre Änderung mit der Amplitude gut erkennen. Hier ist auch bei den tiefen Frequenzen ein Trend zu sehen, das Verhalten ordnet sich nach der Lage zu der Frequenz mit maximaler Verstärkung $f_{\text {max }}$ : für die gemessenen tieferen Frequenzen nimmt die Phase mit wachsender Amplitude ab, bei $f_{\max }$ bleibt sie etwa konstant und bei der höheren Frequenz steigt sie an. Wenn diese Tendenz allgemein gilt, liegt die Kurve zu Ma $=0,2$ und $1007 \mathrm{~Hz}$ etwas oberhalb von $f_{\max }$, da die Phase mit $p_{l}^{+}$ zunimmt.

Abb. 2.11 stellt Betrag und Phase des Reflexionsfaktors $r^{+}$in Abhängigkeit von der Schallamplitude dar. Die Frequenzen und Machzahlen sind die gleichen wie in der vorigen Abbildung 2.10. Die Phase ist wieder bei der maximalen Amplitude auf Null gesetzt. Betrag und Phase von $r^{+}$sind unabhängig vom Schalldruck. Für die betrachteten Machzahlen und Frequenzen liegt der Betrag von $r^{+}$zwischen 1,05 für Ma $=0,2$ und $907 \mathrm{~Hz}$ und 1,3 für Ma=0,25 bei 1087 und $1127 \mathrm{~Hz}$. Einen eindeutigen, wenn auch schwachen Trend zeigt nur die Phase von $r^{+}$für Ma $=0,25$ mit der höchsten Frequenz. 


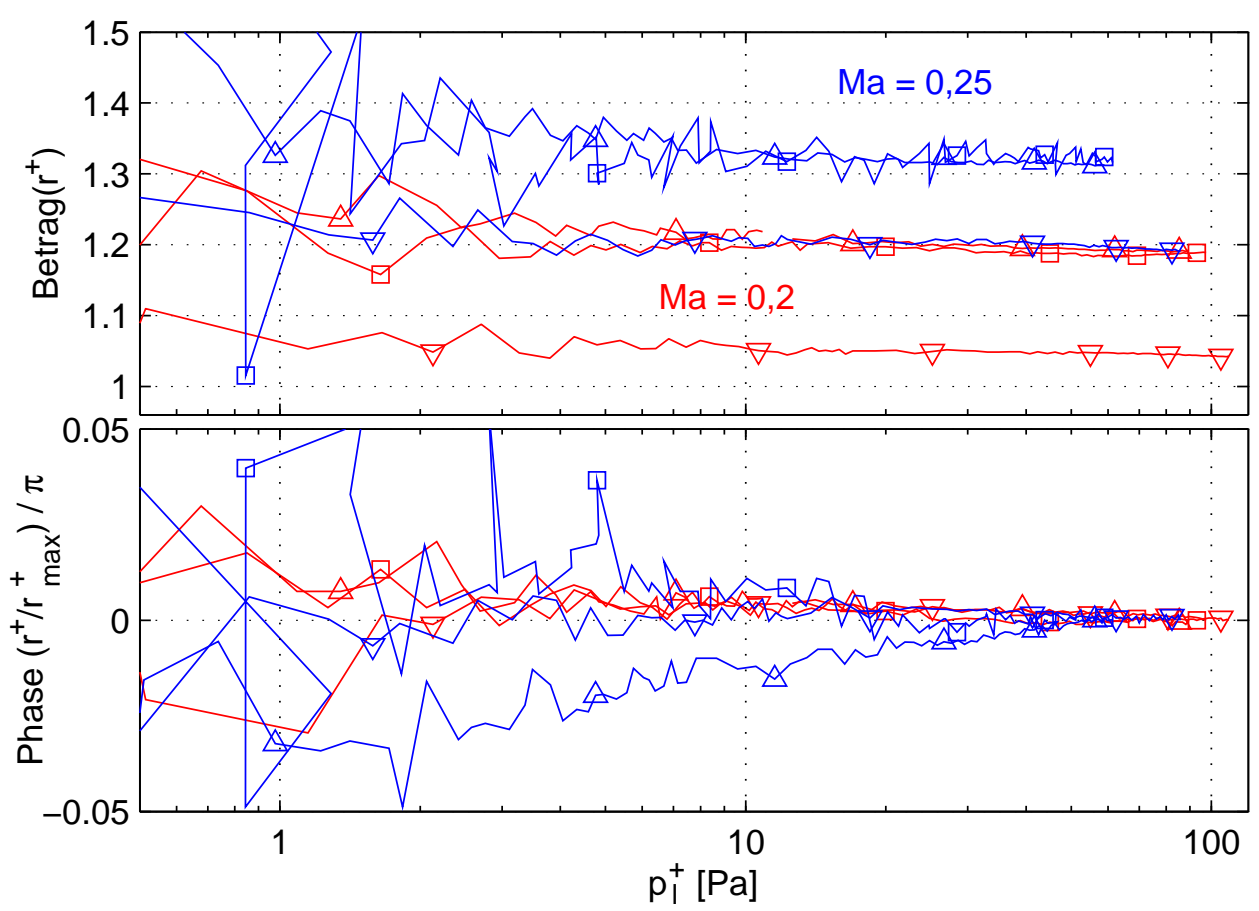

Abb. 2.11: Betrag und Phase des Reflexionsfaktors $r^{+}$als Funktion der Amplitude der einlaufenden Welle, Symbole wie zuvor.

Rot: $\mathrm{Ma}=0,2, \nabla: 907 \mathrm{~Hz}, \square: 1007 \mathrm{~Hz}, \triangle: 1087 \mathrm{~Hz}$, blau: $\mathrm{Ma}=0,25, \nabla: 1007 \mathrm{~Hz}, \square: 1087 \mathrm{~Hz}, \triangle: 1127 \mathrm{~Hz}$.

\section{Beschallung aus dem Resonator stromauf}

Die bisher gezeigten Streufaktoren $t^{+}$und $r^{+}$sind für eine Beschallung mit dem Lautsprecher im Rohr berechnet. Daneben besteht die Möglichkeit, drei Lautsprecher an der stromauf liegenden Resonatorkammer anzubringen. Hier können die Streufaktoren nicht mehr berechnet werden, da die Amplitude der einlaufenden Schallwelle $p_{l}^{+}$nicht gemessen werden kann. Allerdings wird eine Welle $p_{r}^{+}$stromab transmittiert, sowie $p_{l}^{-}$stromauf gestreut. Das Verhältnis ist mit $s^{+}:=p_{r}^{+} / p_{l}^{-}$bezeichnet in Abb. 2.12 aufgetragen. Mit der Annahme, dass der Reflexionsfaktor $r^{+}$zwischen den Lautsprechern und den weiteren Resonatorkammern wie bei der Beschallung aus dem Rohr unabhängig vom Schalldruck ist und nahe bei eins liegt, entspricht die Auftragung also etwa dem Transmissionsfaktor, genauer $t^{+} / r^{+}$. Die Amplitude ist hier nicht mehr absolut geeicht. Sie ist in Einheiten der Ansteuerung $A_{s}$ angegeben, die Kurven sind untereinander damit schlechter vergleichbar. Neben den Werten für Ma $=0,25$ und 0,2 sind hier zusätzlich Messwerte für $\mathrm{Ma}=0,1$ aufgetragen, sowie Messergebnisse mit elf statt 16 offenen Resonatoren (gestrichelt gezeichnet). Die Frequenzen weichen 


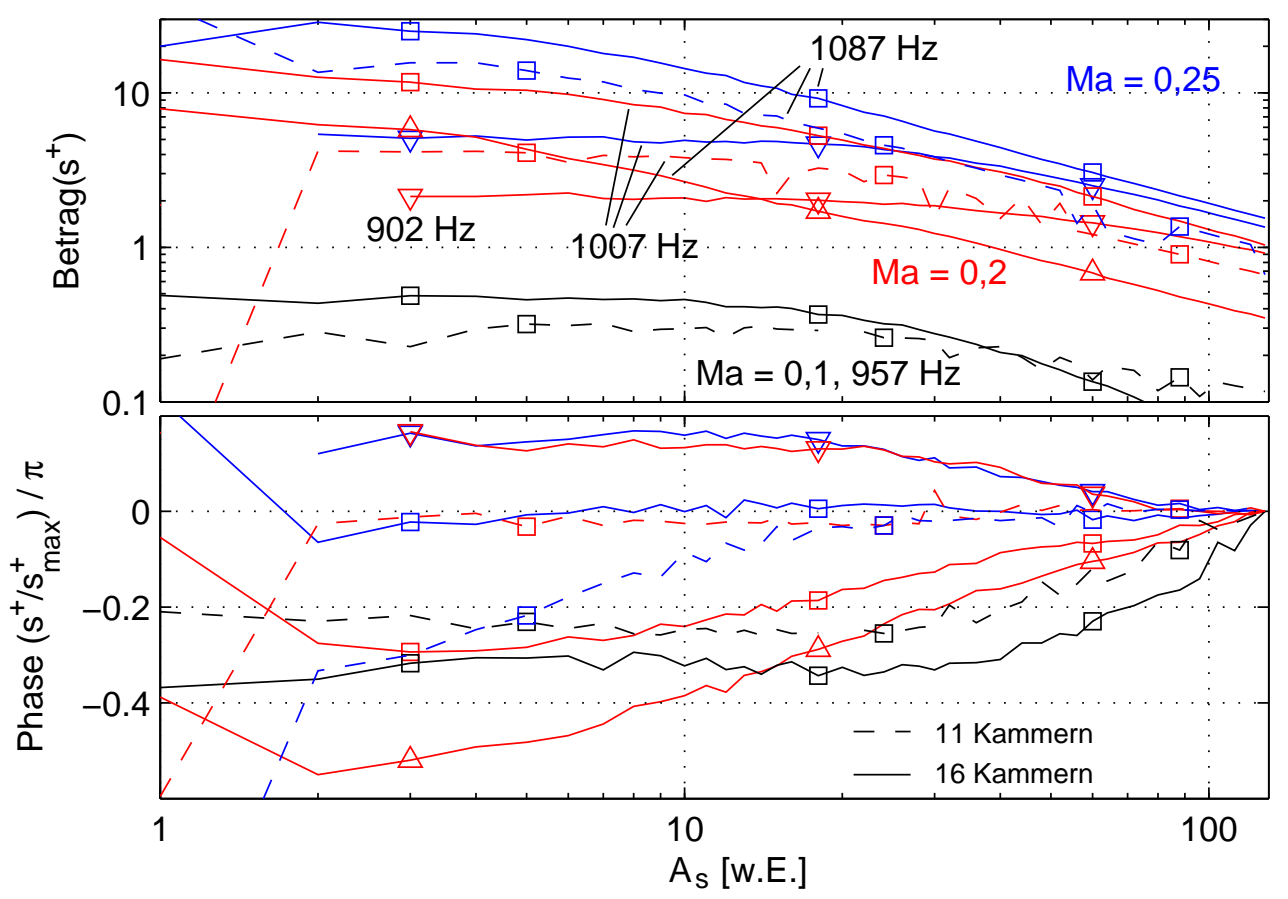

Abb. 2.12: Komplexes Verhältnis $s^{+}$von der stromab zu der stromauf abgestrahlten Schallamplituden (Schätzung des Transmissionsfaktors $t^{+}$) als Funktion der Druckamplitude der stromauf laufenden Welle für Ma =0,25, 0,2 und 0,1 mit verschiedene Frequenzen bei Beschallung aus der ersten von elf bzw.

16 Resonatorkammern.

teilweise von den zuvor gezeigten ab.

Es gibt einige Änderungen von $s^{+}$gegenüber $t^{+}$. So beginnt die Sättigung im Vergleich zur Maximalamplitude schon bei den kleinsten Amplituden. Zudem ist für kleine Amplituden der Betrag von $s^{+}$deutlich größer, als etwa mit der Beziehung $s^{+} \approx t^{+} / r^{+}$ $\mathrm{zu}$ erwarten wäre, beispielsweise bei $\mathrm{Ma}=0,2$ mit $902 \mathrm{~Hz}$ um den Faktor 4 und bei $1007 \mathrm{~Hz}$ um den Faktor 2.

Für Ma $=0,1$ und $957 \mathrm{~Hz}$ ist $s^{+}$bis zu relativ großen Amplituden von etwa $A_{s}=20$ konstant, nimmt dann aber ebenfalls ab. Mit 16 Kammern und oberhalb $A_{s}=40$ nimmt sogar bei steigender Beschallungsamplitude die transmittierte Welle absolut ab (also ohne Normierung auf die Eingangsamplitude). Hier liegt vermutlich eine Instabilitätswelle vor, die nur im Spektrum noch nicht zu erkennen war. Mit der Schallamplitude läuft der Schalldruck nicht einfach in eine Sättigung. Wahrscheinlich ändert sich das zeitlich gemittelte Strömungsprofil mit der Schallamplitude bei dieser Frequenz. Dadurch wird wiederum die Schallausbreitung im Verlauf des geänderten Strömungs- 
profils beeinträchtigt.

Da die Phase mit wachsender Amplitude schließlich ansteigt liegt nach deren Verlauf zu urteilen die Anregungsfrequenz von $957 \mathrm{~Hz}$ etwas oberhalb von $f_{\max }$.

Wesentlich ist aber der große Bereich mit konstantem $s^{+}$(bzw. $t^{+}$, nicht gezeigt). Der Transmissionsfaktor für kleine Strömungsgeschwindigkeiten kann somit bei den kleineren Amplituden der Lautsprecher im Messrohr einfach mit dem von Brandes [1] und Enghard [8] aufgebauten Verfahren gemessen werden.

Die Ursachen für die Unterschiede zwischen $t^{+}$und $s^{+}$müssen an den verschiedenen Lautsprecheranregungen liegen. So ist die Amplitude bei der Anregung aus der Resonatorkammer größer. Hier werden drei Lautsprecher verwendet, die mit 8 $\Omega, 100$ Watt spezifiziert sind. Dagegen befindet sich ein mit $4 \Omega, 100$ Watt spezifizierter Lautsprecher im Rohr. Angetrieben werden sie mit einem Konstant-Strom Verstärker. Nach einer sehr einfachen Abschätzung gibt der Lautsprecher im Rohr demnach 1/6 der Leistung der Anordnung mit drei Lautsprechern ab. Direkt vergleichen lässt sich die Anregungsamplitude jedoch nicht, da die Lautsprecher frequenzabhängig an unterschiedliche Impedanzen angeschlossen sind und unterschiedliche Frequenzgänge haben.

Neben der unterschiedlich eingebrachten Leistung ist die Art der Anregung verschieden. Bei der Anregung aus dem Rohr läuft eine ebene Schallwelle in den Resonatorabschnitt. Zunächst sind Schalldruck und Schallschnelle über dem Rohrquerschnitt konstant. Bei der Anregung aus der Resonatorkammer liegt in der Rohrmitte eine vergleichsweise kleine Schallschnelle vor. Aufgrund der tiefen betrachteten Frequenzen sollte dieser Unterschied nicht weiter als einen Rohrradius in den Resonatorabschnitt hinein reichen.

Es ist möglich, dass dadurch, sowie durch die größere Amplitude bei der Beschallung aus den Resonatorkammern eine Sättigung schon weiter stromauf einsetzt. Möglicherweise lässt sich für die Schallanregung aus dem Rohr durch einen längeren Resonatorabschnitt im Frequenzbereich der Instabilität ein quantitativ ähnliches Verhalten erzeugen wie mit einem kürzeren Abschnitt bei der Anregung aus der Resonatorkammer.

\subsubsection{Frequenz- und Machzahlabhängigkeit von $t^{+}$}

Der Strömungskanal ist für die Messung von akustischen Streufaktoren optimiert. Die Messmethode setzt ein Messobjekt voraus, dessen Streufaktoren unabhängig von der Amplitude der Lautsprecher sind. In diesem Aufbau ist diese Voraussetzung häufig nicht erfüllt, die Abb. 2.10 und 2.12 zeigen die Nichtlinearität. 


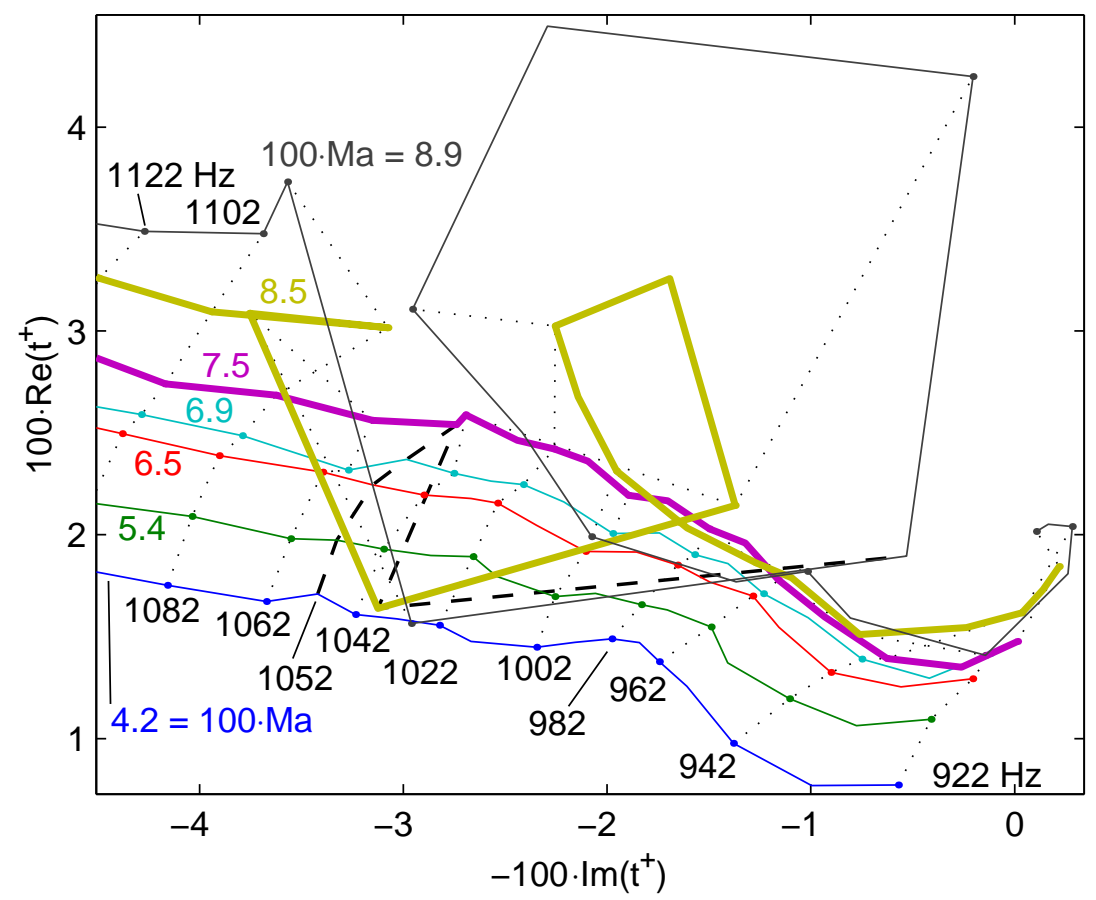

Abb. 2.13: Transmissionsfaktor in Strömungsrichtung $t^{+}$in der (gedrehten) komplexen Ebene für verschiedene Strömungsgeschwindigkeiten im Frequenzbereich um $1050 \mathrm{~Hz}$, in dem die instabile Mode zu sehen ist; der Resonatorabschnitt besteht aus neun Resonatoren.

Bei kleinen Strömungsgeschwindigkeiten und nicht zu großen Amplituden ist keine bedeutende Abhängigkeit der Streufaktoren von der Schallamplitude zu erkennen. Die Streufaktormessung sollte in diesem Fall korrekte Ergebnisse liefern.

Im Folgenden wird gezeigt, wie sich die Instabilitätswelle bei niedrigen Strömungsgeschwindigkeiten auf den Transmissionsfaktor auswirkt. Da bei 16 Resonatoren $t^{+}$ sehr klein wird und Unterschiede leicht im Rauschen untergehen, ist die Messung mit neun offenen Resonatoren durchgeführt. Abb. 2.13 zeigt den Transmissionsfaktor der stromab laufenden Schallwelle in der (um $90^{\circ}$ gedrehten) komplexen Ebene. Die gezeigten Frequenzen liegen zwischen 922 und $1122 \mathrm{~Hz}$, die Machzahl reicht von 0,042 bis 0,089. Bis $\mathrm{Ma}=0,069$ verschiebt sich die Kurve lediglich. Die Messung zu Ma = 0,075 sieht sehr ähnlich aus, hier fallen jedoch die Messwerte zu 1052 und $1042 \mathrm{~Hz}$ fast zusammen. Eine deutliche Änderung ist bei Ma = 0,085 zu sehen: Zwischen 1002 und $1082 \mathrm{~Hz}$ durchläuft $t^{+}$eine Schleife. Bei Ma $=0,089$ vergrößert sich diese Schleife und beeinflusst auch einen etwas größeren Frequenzbereich. Bei kleinen Machzahlen beginnt die Instabilität bei Frequenzen oberhalb der Resonanz. 


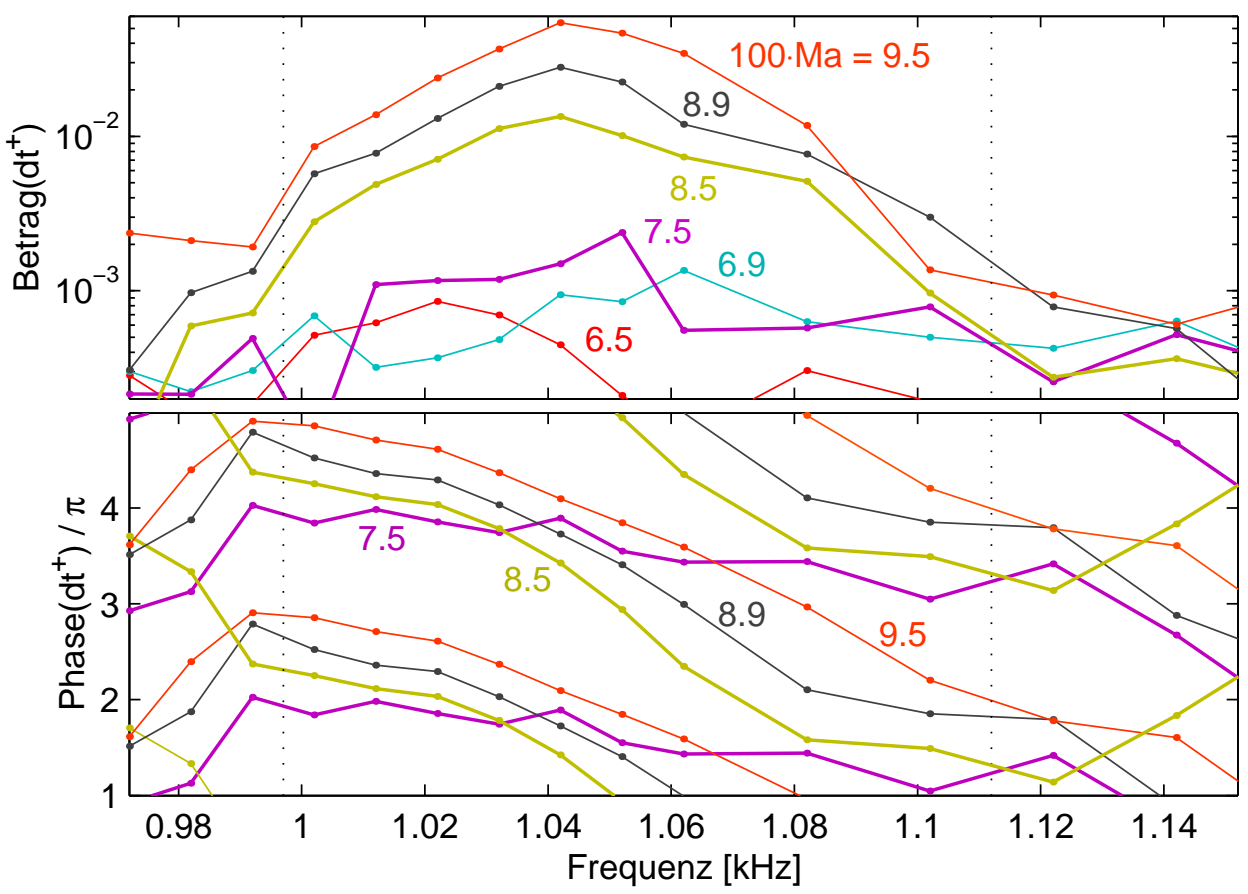

Abb. 2.14: Betrag und Phase der aus $t^{+}$rekonstruierten Amplitude der Instabilitätswellen des Resonatorabschnitts mit neun Kammern.

Die (nicht gezeigten) Reflexionsfaktoren im nicht durchströmten Rohr liegen im betrachteten Bereich relativ konstant um 0,9. Für den Schalleinfall von links bzw. von rechts weichen sie etwa um 5\% voneinander ab, was eine Asymmetrie des Messobjekts anzeigt.

Um die Instabilität deutlicher herauszustellen wird im unbeeinflussten Bereich (je drei Frequenzen um 982 und $1142 \mathrm{~Hz}$ ) ein Polynom 2. Grades angepasst. Der Betrag und die Phase der Differenz zwischen diesem Polynom und den Messwerten $d t^{+}$ist in Abb. 2.14 aufgetragen. Die Frequenzbereiche, die für die Anpassung des Polynoms verwendet wurden, liegen in Abb. 2.14 außerhalb der beiden gestrichelten Linien. Der Betrag von $d t^{+}$in diesen Bereichen zeigt den Anpassungserfolg, die Phase streut dort um das angepasste Polynom.

In der Instabilität (zwischen den gestrichelten Linien) sieht man deutlich den sprunghaften Anstieg zwischen $\mathrm{Ma}=0,075$ und 0,085. Mit steigender Machzahl nimmt der Betrag weiter zu. Die Phase durchläuft dabei etwas mehr als $2 \pi$. Die Phasen für die beiden niedrigsten Machzahlen sind nicht eingezeichnet, um die Abbildung übersichtlich zu halten.

Mit wachsender Strömungsgeschwindigkeit erreicht die Instabilitätswelle größere Am- 
plituden. Es wird schließlich mehr Schall transmittiert als über den Lautsprecher eingebracht. Dabei wird der von der Instabilität beeinflusste Frequenzbereich größer.

Für 16 Resonatoren zeigt Abb. 2.15 den Betrag des Transmissionsfaktor für verschiedene größeren Strömungsgeschwindigkeiten als Funktion der Frequenz. Bei dieser Messung wurde die oben gezeigte Nichtlinearität nicht berücksichtigt, daher sind die Ergebnisse als untere Abschätzung von $\left|t^{+}\right|$zu sehen. Ein Vergleich mit dem Spektrum stromab (Abb. 2.6) zeigt, dass die Überhöhung im Spektrum wie erwartet gut mit dem Frequenzbereich der Instabilität im Transmissionsfaktor übereinstimmt. Die Abweichungen betreffen hauptsächlich den Beginn der Instabilität bei kleinen Machzahlen. So ist im Spektrum noch nichts zu erkennen, wenn $\left|t^{+}\right|$bei Ma $=0,1$ leicht aus dem typischen resonanzartigen Verlauf herausragt. Bei Ma $=0,15$ ist $\left|t^{+}\right|$knapp unterhalb 1, im Spektrum ist eine deutliche Erhebung zu sehen. Bei den größten Machzahlen, wenn die konvektive in eine globale Instabilität übergeht, wird stromab möglicherweise nicht mehr Schallleistung abgestrahlt, sondern nur die selbsterregte Instabilität nahe ihrer Eigenfrequenz mit der des eingebrachten Schalls synchronisiert.

Es sei darauf hingewiesen, dass der Transmissionsfaktor im Bereich der Instabilität von Details am Messobjekt, der Strömung und der Anregung abhängen kann. So hat Brandes mit demselben Resonatorabschnitt an demselben Kanal teilweise deutlich größere Transmissionsfaktoren gemessen [2, Abb. 4.1a]. Für Ma = 0,1 liegt bei $957 \mathrm{~Hz}$ sein Wert um den Faktor 10 über dem hier gezeigten. Vollständig lässt sich dieser Unterschied weder durch die Nichtlinearität von $t^{+}$noch durch eine unterschiedlich bestimmte Machzahl erklären. Das qualitative Verhalten ist jedoch gleich.

Bisher wurde die 2. Resonanz der Kammern um 3,4 kHz nicht weiter beachtet (vgl. die Wandadmittanz der Kammern, Abb. 2.4). Im Spektrum der Abb. 2.6 (Bereiche C und D) ist an dieser Stelle nur für große Machzahlen ab 0,27 eine kleine Überhöhung zu sehen. Bis $\mathrm{Ma}=0,35$ wird es aber durch die 2. Harmonische der bisher betrachteten Instabilität überdeckt, so dass das Einsetzen der Instabilität nicht ausgemacht werden kann.

In Abb. 2.16 ist der Betrag von $t^{+}$im Bereich der 2. Resonanz gezeigt. Ohne Strömung wird $\left|t^{+}\right|$nicht ganz so klein wie in der 1. Resonanz, was aufgrund der Frequenzabhängigkeit der Dämpfung im Resonator auch zu erwarten ist. Mit Strömung sind die Werte recht verrauscht, wachsen aber für Ma $=0,25$ und 0,3 ganz ähnlich wie in der 1. Resonanz an. Das starke Rauschen hat wahrscheinlich zwei Ursachen. Zum einen ist die Amplitude, die der Lautsprecher in diesem Frequenzbereich erzielen kann, relativ gering. Zum Anderen sind im Resonatorabschnitt bei Frequenzen oberhalb von $3 \mathrm{kHz}$ zahlreiche nicht-radialsymmetrische Moden ausbreitungsfähig, die durch Asymmetrien im Aufbau und insbesondere in den turbulenten Fluktuationen angeregt werden 


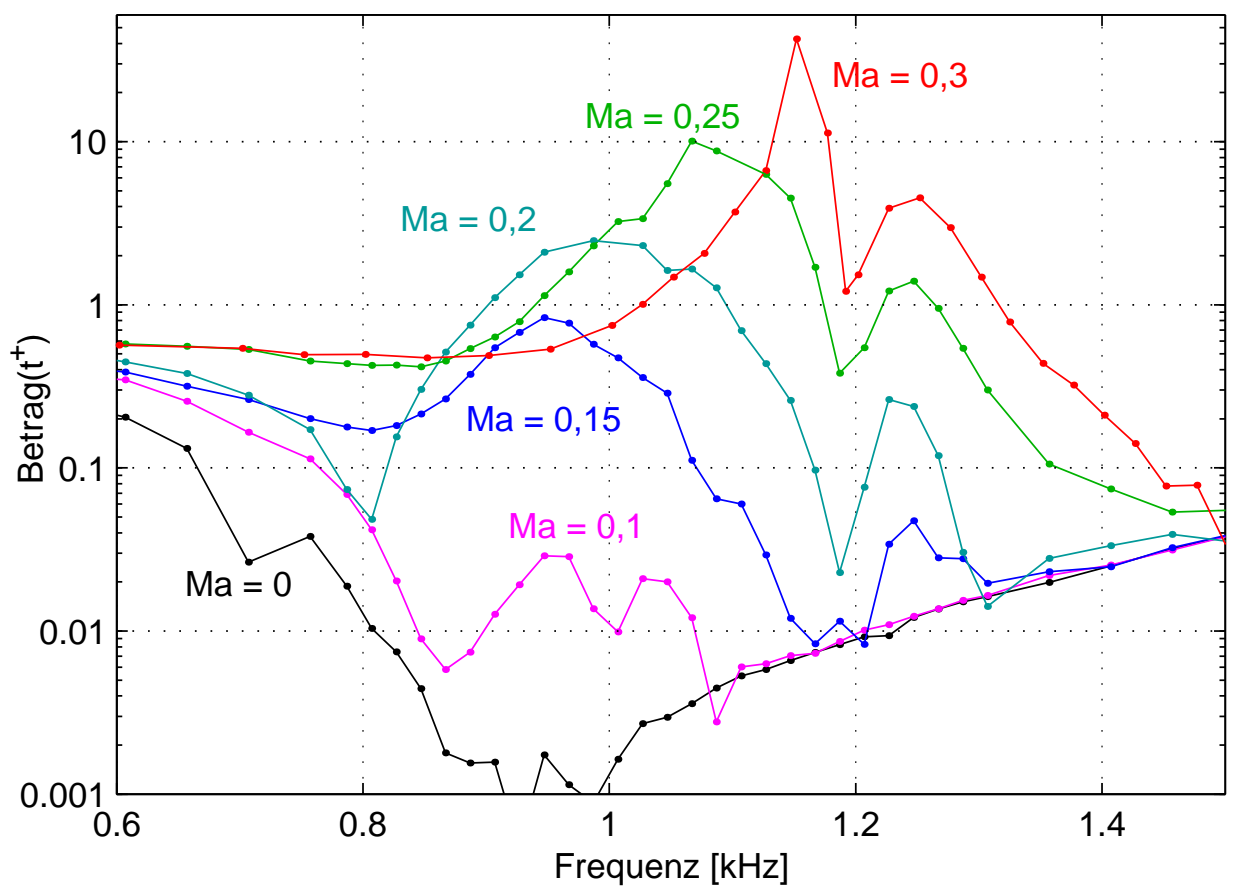

Abb. 2.15: Transmissionsfaktor $t^{+}$des Resonatorabschnitts mit 16 Kammern für Frequenzen im Bereich der ersten Kammerresonanz bei verschiedenen Machzahlen.

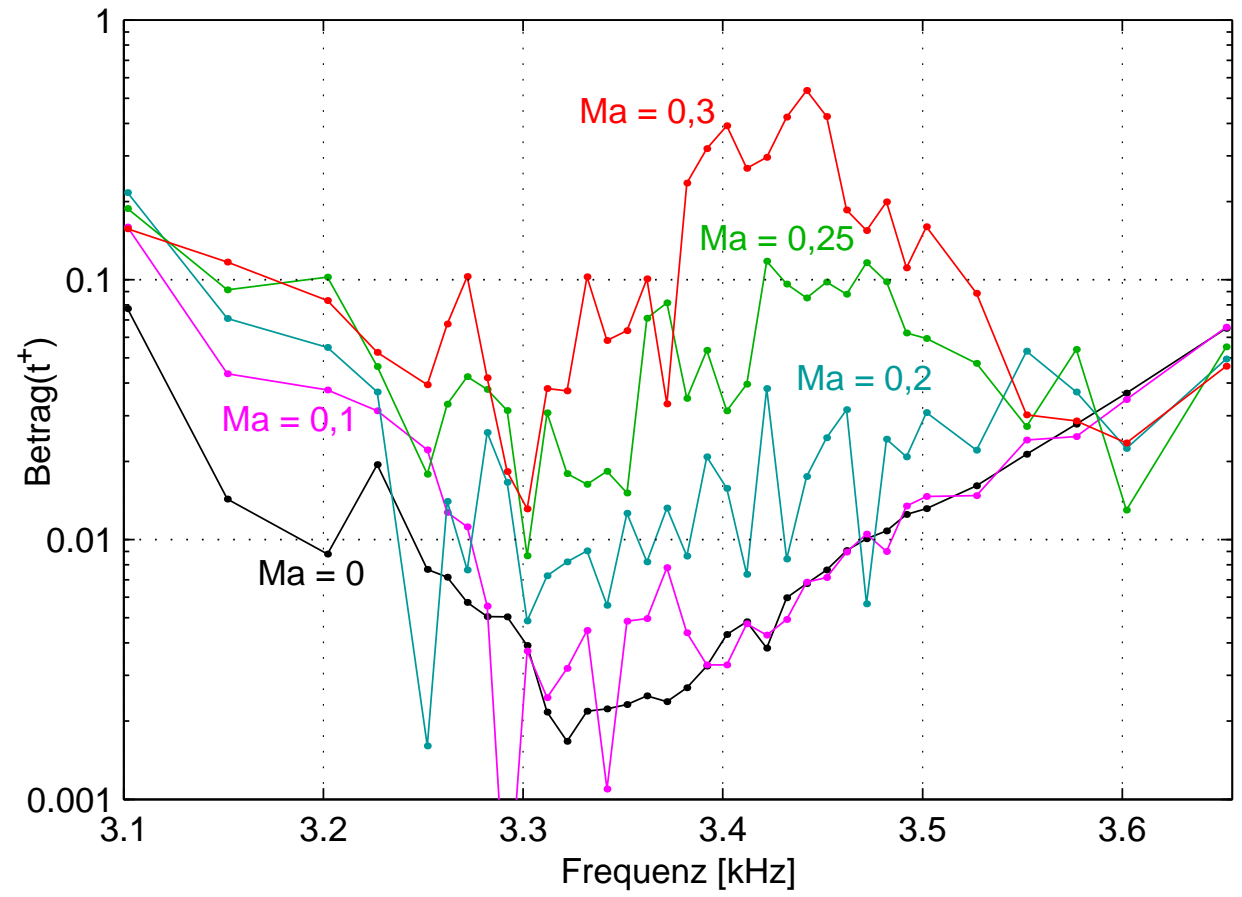

Abb. 2.16: Transmissionsfaktor $t^{+}$des Resonatorabschnitts mit 16 Kammern für Frequenzen im Bereich der zweiten Kammerresonanz bei verschiedenen Machzahlen. 


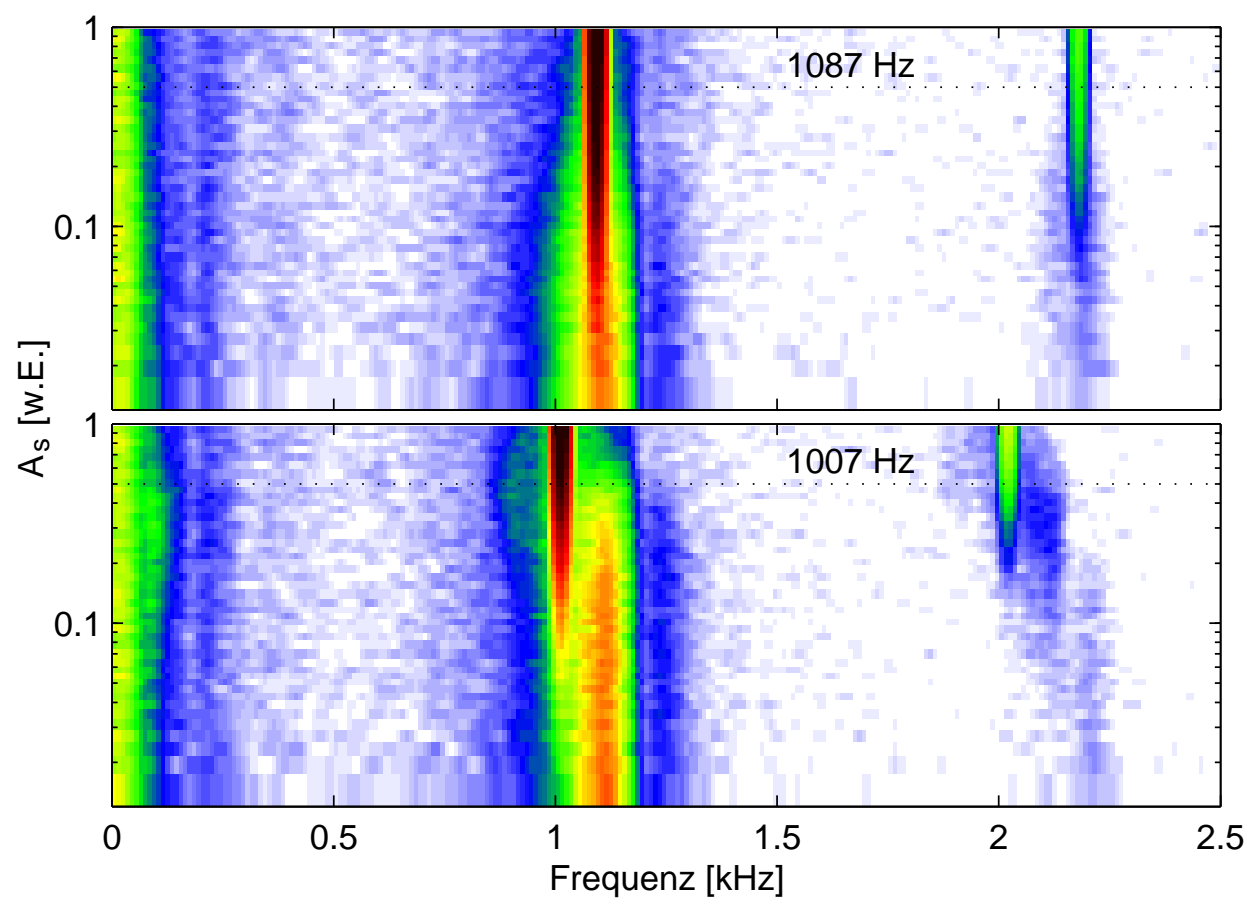

Abb. 2.17: Spektrum stromab des Abschnitts mit 16 Resonatoren bei Ma $=$ 0,25 in Abhängigkeit von der Anregungsamplitude, Beschallung aus dem Rohr stromauf mit $1087 \mathrm{~Hz}$ (oben) und $1007 \mathrm{~Hz}$ (unten).

können und so die radialsymmetrische Instabilität beeinflussen, siehe Abschnitt 2.6.

\subsubsection{Spektren bei monofrequenter Beschallung}

\section{Einfluss der Beschallungsamplitude}

Von hier ab wird das Druckspektrum nur im Rohr stromab betrachtet. Der Schall beeinflusst das Spektrum nicht nur bei der eingebrachten Frequenz sondern in einem größeren Bereich. Das Spektrum ist in Abb. 2.17 abhängig von der Beschallungsamplitude $A_{s}$ für $\mathrm{Ma}=0,25$ bei den Beschallungsfrequenzen $f_{s}=1087 \mathrm{~Hz}$ und $f_{s}=1007 \mathrm{~Hz}$ aufgetragen. Die Werte von $0 \mathrm{~dB}$ bis $40 \mathrm{~dB}$ über dem Median der jeweiligen Messung sind farbcodiert, siehe Farbtabelle 0.1. Die unterste Zeile der Spektren zeigt jeweils eine Messung ohne Lautsprecheranregung.

Sowohl für $f_{s}=1087 \mathrm{~Hz}$ als auch für die 2 . Hamonische um $2 f_{s} \approx 2,2 \mathrm{kHz}$ ist ab $A_{s}=0,05$ ein Einfluss auf das Spektrum erkennbar; bei diesen Frequenzen tritt im Spektrum Verstärkung auf. 
Um $A_{s}=0,1$ nimmt die Breite des Instabilitätsbereichs ab. Hier tritt die Spektrallinie der eingebrachten Frequenz deutlicher hervor. Die Energie in den Druckschwankungen wird also zunehmend auf die Anregungsfrequenz konzentriert. Bei wenig größeren Amplituden $A_{s}$ nimmt auch die Breite des Bereichs um die 2. Hamonische ab.

Für $f_{s}=1007 \mathrm{~Hz}$ ist ebenfalls ab $A_{s}=0,05$ ein Anwachsen der Spektrallinie zu sehen. Damit wird die Überhöhung um die 2. Harmonische beeinflusst, die sich von $2 f_{\max } \approx 2,2 \mathrm{kHz}$ auf $f_{\max }+f_{s} \approx 2,1 \mathrm{kHz}$ verlagert. Ab $A_{s}=0,15$ kommt bei $2 f_{s}$ die 2. Harmonische der Beschallungsfrequenz hervor. Bei ähnlichen Amplituden wird allmählich das Spektrum oberhalb von $f_{s}$ bis $1,3 \mathrm{kHz}$ flacher. Zudem tritt die Differenzfrequenz zwischen der turbulent und der mit dem Lautsprecher angeregten Frequenz selbst $\left|f_{\max }-f_{s}\right|$ auf (siehe auch Abb. 2.18). Bei größeren Amplituden konzentriert sich die Schallleistung zunehmend bei der Anregungsfrequenz.

Die waagerechte gepunktete Linie in Abb. 2.17 bei der Amplitude 0,5 zeigt den Wert an, bei dem die folgenden Messungen durchgeführt wurden. Bei diesen Amplituden sind also schon nichtlineare Effekte im Spektrum zu sehen.

\section{Einfluss der Beschallungsfrequenz}

Die Abhängigkeit des Spektrums von der Anregungsfrequenz $f_{s}$ ist für $\mathrm{Ma}=0,25$ in Abb. 2.18 für die beiden vorhandenen Lautsprecherpositionen aufgetragen. Bei der Anregung aus dem Rohr (oberes Teilbild) ist der Einfluss auf den Verstärkungsbereich im Wesentlichen auf Frequenzen zwischen 950 und $1200 \mathrm{~Hz}$ beschränkt. Hier konzentriert sich der größte Teil der Schallenergie auf die Anregungsfrequenz. Die Überhöhung, die ohne Schall vorhanden ist, wird in diesem Frequenzbereich nahezu vollständig unterdrückt. Die eingebrachte Frequenz selbst sowie die Differenzfrequenz $\left|f_{\max }-f_{s}\right|$ ist für $f_{s}$ zwischen 0,9 und $1,3 \mathrm{kHz}$ zu sehen.

Bei der Beschallung aus der Resonatorkammer (unteres Teilbild) ist über den gesamten gezeigten Bereich der Einfluss des Schalls auf das übrige Spektrum zu sehen. Bei Frequenzen bis $f_{s}=1,35 \mathrm{kHz}$ ist der größte Teil der Energie um die eingebrachte Frequenz konzentriert. Aber auch bei höheren Frequenzen flacht der Instabilitätsbereich deutlich gegenüber dem Fall ohne Beschallung ab. Die Harmonische um $2 f_{\max } \approx 2,2 \mathrm{kHz}$, die bei Beschallung aus dem Rohr für Frequenzen deutlich unterhalb bzw. oberhalb der Instabilität zu sehen ist, tritt hier nicht hervor - selbst dann nicht, wenn die Anregungsfrequenz nicht mehr im Spektrum zu erkennen ist, wie hier oberhalb von 1,5 kHz. Unterhalb von $0,7 \mathrm{kHz}$ tritt zunehmend die zweite Harmonische der Anregungsfrequenz in den Verstärkungsbereich und beeinflusst wieder das Spektrum, daher ist die Darstellung hier abgeschnitten. 


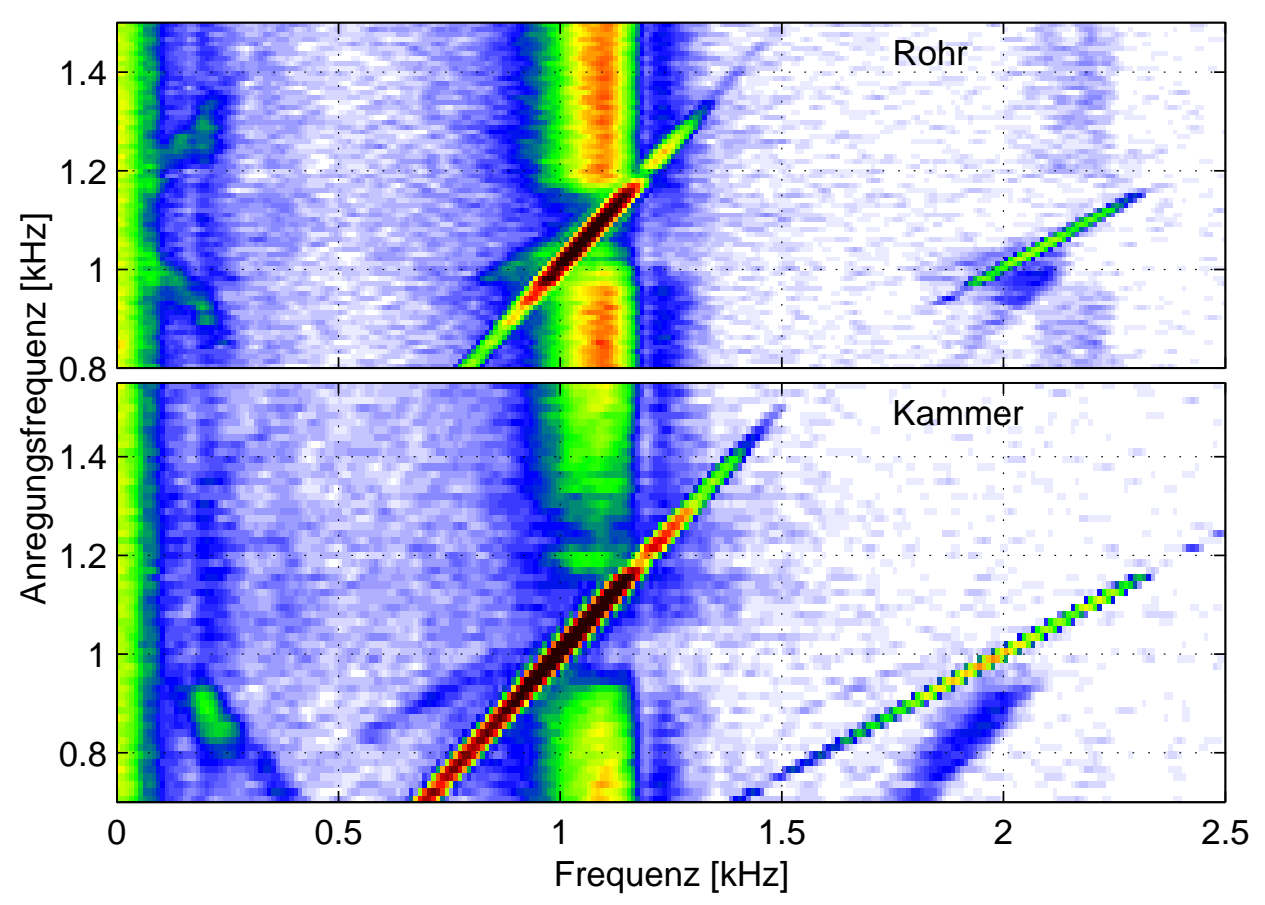

Abb. 2.18: Spektrum stromab des Abschnitts mit 16 Resonatoren bei Ma $=$ 0,25 in Abhängigkeit von der Anregungsfrequenz. Beschallung aus dem Rohr (oben) und aus der Resonatorkammer (unten).

Die spektrale Verteilung der Energie der Druckschwankungen stromab des Resonatorabschnitts im Bereich der Instabilität soll etwas näher betrachtet werden, siehe Abb. 2.19. Dazu ist der Frequenzbereich von 0,7 bis $1,5 \mathrm{kHz}$ herausgegriffen und die Energie in Abhängigkeit von der Beschallungsfrequenz aufgetragen (schwarze Kurve). Die Kurven sind auf die Energie ohne Beschallung normiert. In den beiden weiteren Kurven ist die Energie aufgeteilt in den Bereich unmittelbar um die Beschallungsfrequenz (rot) und den übrigen Bereich (blau), so dass deren Summe wieder die gesamte Energie im betrachteten Bereich angibt. Die durchgezogenen Linien gelten für eine Beschallung aus der ersten Resonatorkammer. Die gestrichelten Linien zeigen gleiches für eine Beschallung aus dem Rohr stromauf.

Bei der Anregung aus dem Rohr verlaufen die Kurven weitgehend wie erwartet: tiefe Frequenzen unterhalb $900 \mathrm{~Hz}$ beeinflussen die Instabilität nicht, die Energieverteilung bleibt unverändert. Mit steigender Frequenz nimmt der Einfluss auf die Instabilität zu, zwischen 950 und $1170 \mathrm{~Hz}$ ist nahezu die gesamte Schallenergie in der angeregten Frequenz. In diesem Bereich wird nicht nur die aufgeprägte Schallwelle verstärkt, sondern auch die Energie aus dem benachbarten Bereich abgezogen, wie es schon in Abb. 2.18 zu sehen war. Hier kommt das durch die blaue Kurve „Rest“ zum Ausdruck, die 


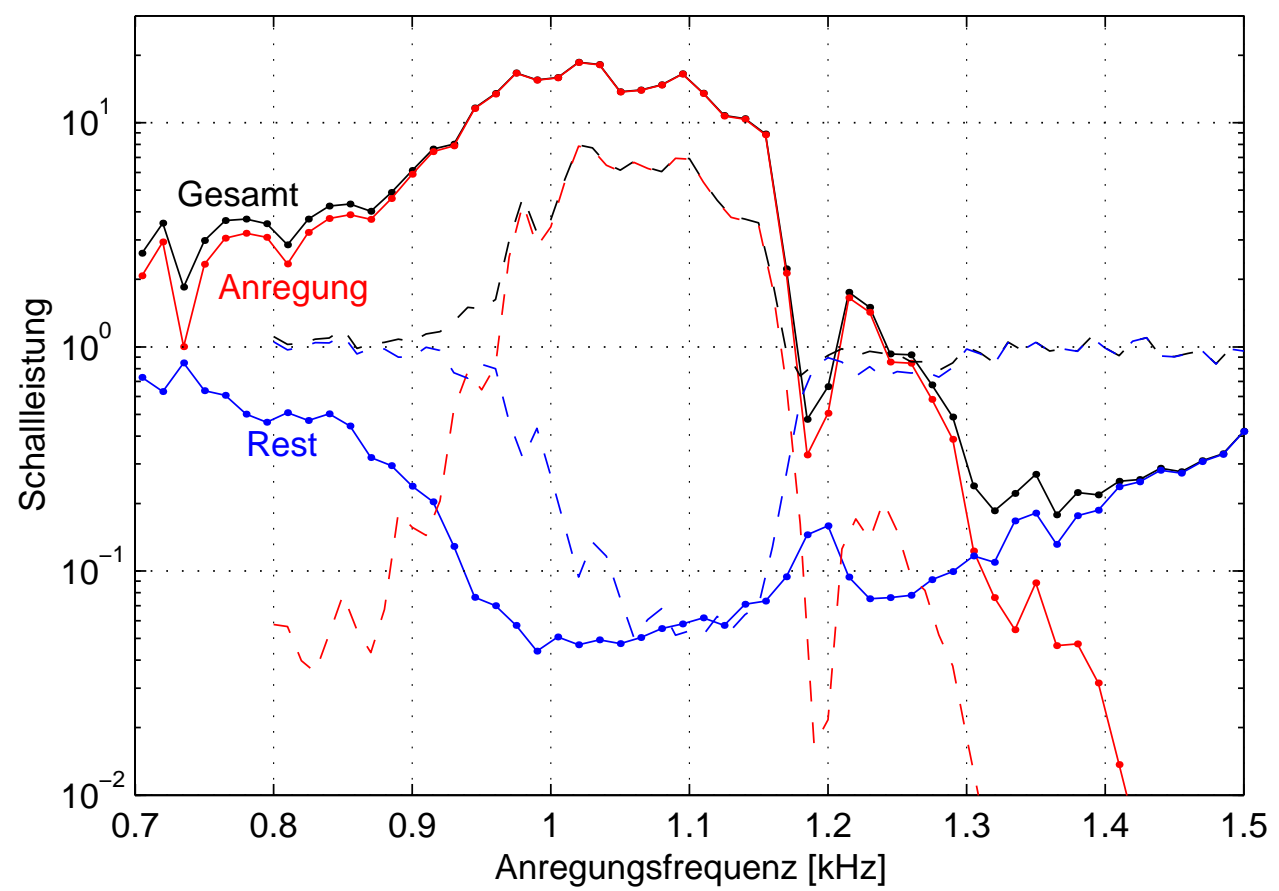

Abb. 2.19: Leistung der Druckschwankungen stromab bei Ma $=0,25$ zwischen 0,7 und $1,5 \mathrm{kHz}$ in Abhängigkeit der Anregungsfrequenz, Beschallung aus dem Resonator (durchgezogene Linie) und aus dem Rohr (gestrichelt), sowie aufgeteilt in Frequenzen um die Anregung (rot) und den übrigen Bereich (blau), normiert auf die Leistung ohne Beschallung.

dann deutlich unter dem Normierungswert ohne Schallquelle liegt. Frequenzen oberhalb $1170 \mathrm{~Hz}$ beeinflussen die Instabilität kaum und sind selbst im Spektrum immer weniger zu erkennen.

Bei der Beschallung aus der Resonatorkammer ist schon bei tiefen Frequenzen der eingebrachte Schall dominant. Bis zu 1,3 kHz bleibt die meiste Energie auf den engen Spektralbereich um die Anregungsfrequenz konzentriert. Auch darüber ist der Einfluss der Anregung daran zu erkennen, dass die Gesamtenergie kleiner bleibt als ohne Lautsprecheranregung. Die gesamte abgestrahlte Schallenergie kann hier sowohl vergrößert, als auch verkleinert werden. Es wird nochmal deutlich, dass sich bei allen Frequenzen das Spektrum ändert.

Zwischen den beiden Beschallungsarten bestehen drei Unterschiede, auf die hingewiesen werden soll. Erstens ist die Lautsprecheramplitude verschieden. Damit kann die Differenz bei den Anregungen aber nicht erklärt werden, denn in den Randbereichen, in denen das System vergleichsweise linear reagiert, ist die Abweichung besonders groß. 
Zweitens ist die Anregung aus dem Rohr bis auf turbulente Druckschwankungen radialsymmetrisch. Die drei Lautsprecher an der Resonatorkammer können dagegen nicht exakt gleichphasig und mit gleicher Amplitude betrieben werden, siehe Abschnitt 2.6.2.

Schließlich regen die Lautsprecher im Resonator ein anderes Druck- und Schnellefeld an, das die Instabilität auch bei tieferen Frequenzen noch beeinflussen könnte. Hier wird etwa der Wechseldruck in der Kanalmitte wesentlich kleiner sein, als bei dem Einfall einer ebenen Welle. Mathematisch ließe sich das in einer anderen Wahl der Randbedingungen beim Übergang zwischen Rohr und Resonatoren berücksichtigen, siehe Großer [10, Kap. 5].

Offenbar gibt es nur einen begrenzten Vorrat an Energie, der in die instabilen Frequenzen transferiert werden kann. Wäre die Energie unbegrenzt, sollten die turbulent angeregten Frequenzen nicht durch die Schallanregung bei verschiedenen Frequenzen beeinflusst werden (d. h. die Kurven „Rest" in der Abbildung 2.19 müsste bei eins liegen). Durch die Anregung wird die Ausgangssituationen für die verschiedenen Frequenzen geändert, so dass ggf. auch die Druckschwankungen um $f_{\max }$ nur vergleichsweise schwach verstärkt werden.

Die Energie, die zum Anstieg der akustischen Drücke führt, kann (neben dem Lautsprecher) nur aus der Gleichströmung kommen. Die Energie aus dem Lautsprecher alleine kann natürlich nicht für eine Schallverstärkung verantwortlich sein, da die Schallenergie vor dem Resonatorabschnitt kleiner ist als dahinter.

Wenn die Instabilität mit einer Frequenz angeregt wird, die verstärkt abgestrahlt wird, muss Energie aus der Strömung in den Schall übergehen oder zumindest Energie aus anderen Frequenzbereichen auf die eingebrachte Frequenz synchronisiert werden. Dass es sich nicht nur um eine Synchronisation handelt, wird anhand der Abbildung 2.15 und 2.19 deutlich.

Welche Frequenzen verstärkt abgestrahlt werden, hängt neben der Anregungsamplitude von der Länge des Resonatorabschnitts ab. Mit zunehmender Amplitude und mit zunehmender Länge verschiebt sich das Maximum zu tieferen Frequenzen.

Die Ursache dieser Verschiebung liegt wahrscheinlich in einer Änderung des Strömungsprofils und der damit verbundenen Turbulenzverteilung. Diese Änderung wird durch die Druckschwankungen verursacht. Kleinere Anregungsamplituden benötigen einen längeren Laufweg, bis sie eine gleichartige Änderung des Strömungsprofils verursachen, wie eine größere Amplitude am Beginn des Resonatorabschnitts, daher sind diese Größen äquivalent. Für tiefere Frequenzen scheint die Änderung des Strömungsprofils einen größeren Laufweg im Resonatorabschnitt zu benötigen, so dass sich das Verstärkungsmaximum bei langen Resonatorabschnitten zu tieferen Frequenzen verschiebt. 
Die Änderung des Strömungsprofils steht im Zusammenhang mit effektiv erhöhten Schubspannungen. Diese führen zu einem erhöhten Druckabfall, der im folgenden Abschnitt näher betrachtet wird.

\subsection{Schallinduzierte Veränderung des Druckabfalls}

An diesem Aufbau wurde erstmals von Krause [14] ein zusätzlicher Druckabfall der Gleichströmung bei eingebrachten Schall beobachtet. Brandes [2, Kap. 4.4] hat gezeigt, dass zwei angeregte benachbarte Frequenzen infolge des modulierten Druckabfalls bei ihrer Schwebungsfrequenz Schall abstrahlen. Schließlich hat Lange [15] den Effekt genutzt, um mit einem aktiven Filter tieffrequente Schallausbreitung im Rohr breitbandig zu kompensieren.

Um diesen schallinduzierten Druckabfall genauer zu bestimmen, muss zunächst der Strömungszustand im Messrohr, insbesondere die Geschwindigkeit, möglichst genau ermittelt werden. Dann wird der Einfluss des Resonatorabschnitts ohne Beschallung durch die Lautsprecher beschrieben, bevor die Abhängigkeit des Druckabfalls von der Beschallungsfrequenz, der Strömungsgeschwindigkeit und der Schallamplitude untersucht wird. Anschließend werden im nächsten Abschnitt die Abhängigkeiten für die Anregung mit einer höheren, nicht abstrahlenden Mode betrachtet.

\subsubsection{Strömungszustand im Rohr}

Der Strömungszustand im Rohr kann nicht direkt in einer Messung mit den vorhandenen Sensoren bestimmt werden. Es fehlt ein Wert, der angibt, welche Dichte bei dem gemessenen Druckabfall am Kanaleinlauf vorliegt. Die möglichen Extreme wären die adiabatische und die isotherme Zustandsänderung. Es muss also eine Eichung erfolgen.

Für die in dieser Arbeit gezeigten Messungen ist aufgrund einer Eichung aus Vorgängerarbeiten die Machzahl über die Beziehung Ma $=0,96 \sqrt{\Delta p_{1} / p_{0}}$ eingestellt worden, wobei $p_{0}$ der atmosphärische Druck im Labor und $\Delta p_{1}$ der Druckabfall am Kanaleinlauf ist. Diese Beziehung ist jedoch zu ungenau, wenn Aussagen über den Druckabfall im Messobjekt gemacht werden sollen, also der Druckabfall im Messrohr abgezogen werden muss. Daher wurde die Abhängigkeit von $\Delta p_{1} / p_{0}$ erneut vermessen. Weitere Parameter, etwa die Temperatur, können nicht eingestellt werden und haben auch nur einen sehr geringen Einfluss auf die Strömungsgeschwindigkeit. 
Zur Eichung bestehen zwei Möglichkeiten: Eine Eichung ist im leeren Rohr über die (über dem Querschnitt gemittelten) Kontinuitäts-, die Energie- und die Rohrreibungsgleichung zusammen mit der idealen Gasgleichung möglich,

$$
\begin{aligned}
m & :=\rho \bar{u}=\text { const. } \\
e & :=\left(\frac{\mathrm{Ma}^{2}}{2}+\frac{1}{\gamma-1}\right) c^{2}=\text { const. } \\
\frac{d\left(p+\rho \bar{u}^{2}\right)}{d x} & =-\frac{\lambda}{D} \frac{\rho}{2} \bar{u}^{2} \\
\gamma \frac{p}{\rho} & =c^{2}
\end{aligned}
$$

Die Rohrreibungzahl $\lambda$ lässt sich aus dem Prandtlschen Widerstandsgesetz für glatte Rohre

$$
\frac{1}{\sqrt{\lambda}}=2,0 \log \left(\frac{u D}{\nu} \sqrt{\lambda}\right)-0,8
$$

berechnen [24].

Das Strömungsprofil wird nicht berücksichtigt. Die Abweichungen durch Produkte von profilabhängigen Größen sind klein gegenüber anderen Unsicherheiten. Die Gleichungen lassen sich so umstellen, dass in der Rohrreibungsgleichung die Machzahl Ma $=\bar{u} / c$ als einzige Variable bleibt, über die anschließend integriert werden kann. So bekommt man den Zusammenhang zwischen den Machzahlen $\mathrm{Ma}_{1}$ und $\mathrm{Ma}_{2}$ an den Orten $x_{1}$ und $x_{2}$ :

$$
\frac{\gamma+1}{2} \log \frac{\left(\mathrm{Ma}_{2}^{2}+\frac{2}{\gamma-1}\right) \mathrm{Ma}_{1}^{2}}{\left(\mathrm{Ma}_{1}^{2}+\frac{2}{\gamma-1}\right) \mathrm{Ma}_{2}^{2}}+\frac{1}{\mathrm{Ma}_{1}^{2}}-\frac{1}{\mathrm{Ma}_{2}^{2}}=\gamma \lambda \frac{x_{2}-x_{1}}{D}
$$

Die noch unbekannten Machzahlen können über

$$
\mathrm{Ma}^{2}=\sqrt{\frac{1}{(\gamma-1)^{2}}+2 e \frac{m^{2}}{\gamma^{2} p^{2}}}-\frac{1}{\gamma-1},
$$

die ebenfalls aus obigen Gleichungen folgt, ersetzt werden.

Der Druck wird an zwei Stellen gemessen, die Konstante $e$ wird aus der Messung der Temperatur an einer Stelle mit $\mathrm{Ma}=0$, mit Korrekturen für die Luftfeuchtigkeit und den atmosphärischen Druck bestimmt [5], so dass als einzige Variable $m$ (und der Rohrreibungskoeffizient $\lambda(m))$ bleibt. Damit kann iterativ $m$ und über Gleichung 2.11 die Machzahl berechnet werden. Die so aus der Strömung gewonnene Machzahl für den Ort, an dem die beiden Teilrohre zusammentreffen ist in Abb. 2.20 normiert auf $\mathrm{Ma}_{\text {ref }}=0,96 \sqrt{\Delta p_{1} / p_{0}}$ über $\mathrm{Ma}_{\text {ref }}$ als rote Kurve eingezeichnet. 


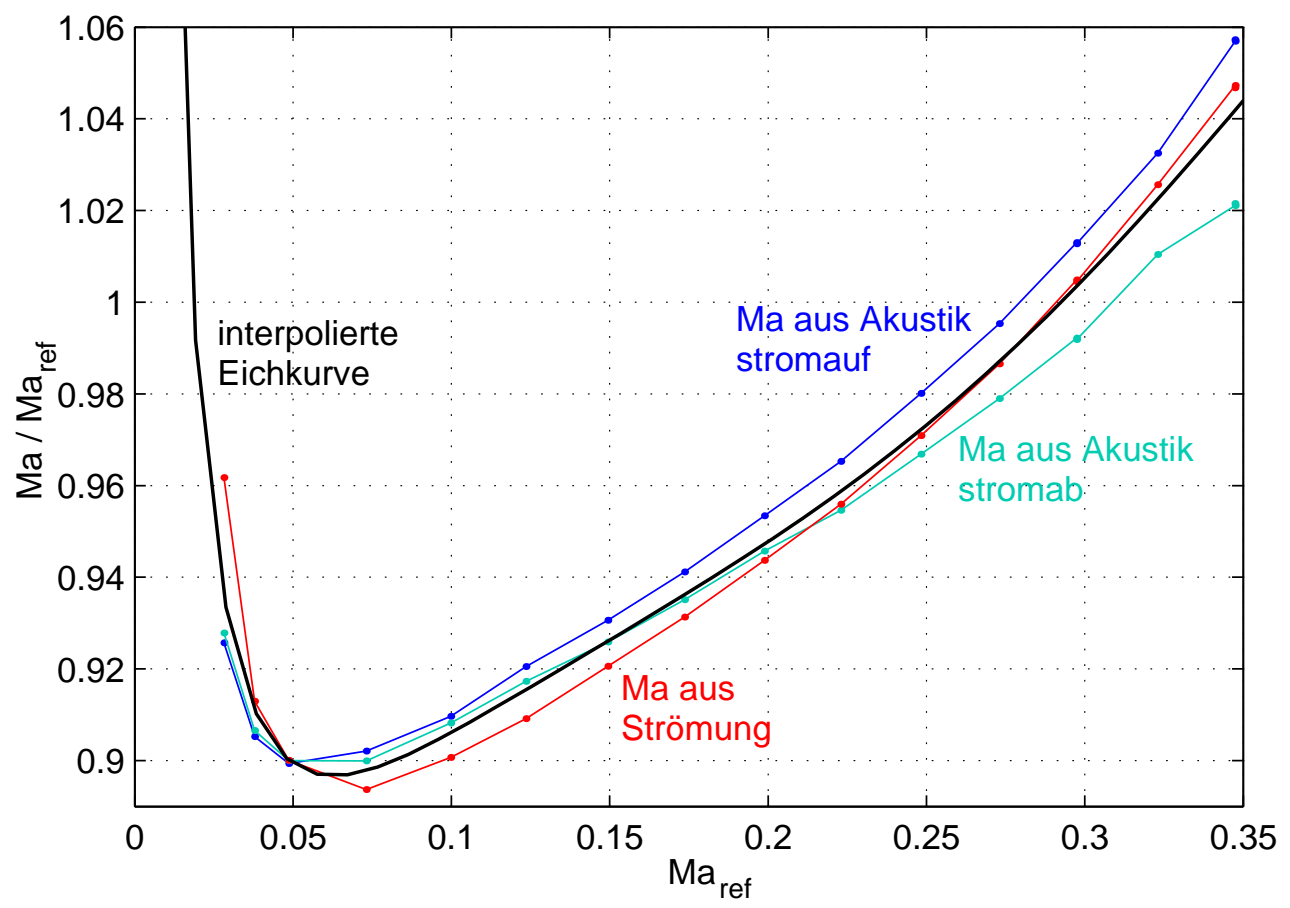

Abb. 2.20: Akustische Machzahlen aus den Teilrohren stromauf und stromab der Auskleidung, und aus der Gleichströmung ermittelte Machzahl.

Die zweite Eichmöglichkeit ist akustisch. Das Rohr wird durch zwei Lautsprecher stromab und stromauf beschallt, Wellenzahlen werden an die Schallfelder angepasst, aus denen in jedem Teilrohr eine akustische Machzahl bestimmt wird. Diese weicht für Strömungen bis $\mathrm{Ma}=0,35$ nach Ronneberger um maximal 0,6\% von der Konvektionsmachzahl ab [23, Abs. 3.2.1]. Damit wird für jedes Teilrohr eine Machzahl an dem Ort ausgegeben, an dem die beiden Teilrohre ohne Messobjekt zusammenstoßen. Sie sollten also gleich sein. Die beiden akustischen Machzahlen sind ebenfalls in Abb. 2.20 eingezeichnet.

Die verschiedenen Machzahlen weichen um bis zu $10 \%$ bei $\mathrm{Ma}=0,075$ von der vorherigen Referenz-Machzahl $\mathrm{Ma}_{\text {ref }}$ ab. Die Abweichung zwischen den drei Kurven beträgt bis $\mathrm{Ma}=0,3$ maximal 2\%, allerdings ist der Verlauf aller Kurven systematisch unterschiedlich. Die beiden akustischen Machzahlen liegen bei kleinen Strömungsgeschwindigkeiten nahe beieinander und entfernen sich dann zunehmend. Dabei ist die stromab ermittelte Machzahl kleiner als die im Rohr stromauf. Die Strömungsmachzahl ist ab $\mathrm{Ma}=0,05$ die kleinste, nähert sich aber bald wieder den akustisch bestimmten. Bei $\mathrm{Ma}=0,22$ überschreitet sie die aus dem Rohr stromab.

Eine wahrscheinliche Ursache für die unterschiedlichen Machzahlen liegt in der ver- 
wendeten Rohrreibung nach Gleichung 2.9. Bei allen Kurven wird die Prandtlsche Rohrreibung für eine ausgebildete turbulente Strömung vorausgesetzt. Die erste Druckmessstelle liegt $22 D$ stromab des Einlaufendes, an dem das Rohr seinen konstanten Durchmesser von $D=50 \mathrm{~mm}$ erreicht. Nach Schlichting [24, Kap. XX] wird das ausgebildete Strömungsprofil erst zwischen 25 und $100 D$ erreicht.

Zudem ist das Rohr in Strömungsrichtung leicht wellenförmig. Dies könnte zu systematischen Abweichungen von $\lambda$ zum Literaturwert beitragen.

Im Weiteren wird die in Abb. 2.20 schwarz eingezeichnete Kurve als Eichung verwendet. Sie kommt durch Interpolation der drei Messkurven zustande. Bis Ma = 0,275 ist die maximale Abweichung kleiner als $1 \%$.

\subsubsection{Druckabfall am Resonatorabschnitt ohne Beschallung}

Zunächst wird der Einfluss des Resonatorabschnitts auf den Druckabfall gezeigt, ohne dass neben den turbulenten Fluktuationen zusätzlicher Schall durch die Lautsprecher eingebracht wird. Abb. 2.21 stellt die Änderung des Druckabfalls durch den Einbau des Resonatorabschnitts dar. Der Druckabfall $\Delta p$ ist auf den Staudruck am Resonatoreingang $\Delta p_{\text {dyn, offen }}$, ggf. noch vor einem Zentralkörper, normiert und in Abhängigkeit von der Machzahl aufgetragen. Parameter sind die Anzahl offener Resonatorkammern sowie die Breite des freien Ringspaltes. Der Druckabfall wird über die gesamte Rohrlänge bestimmt. Das berechnete $\Delta p$ ist die Differenz des Druckabfalls mit dem Resonatorabschnitt zu einer Referenzmessung ohne diesen Abschnitt.

Der Resonatorabschnitt hat eine Gesamtlänge von $L=118 \mathrm{~mm}$ (inklusive der Halterung). Für ein glattes Rohrstück dieser Länge kann in Gleichung 2.7 die Änderung des Staudrucks vernachlässigt und $d x=L$ gesetzt werden. Als normierten Druckabfall bekommt man somit $\Delta p / p_{\text {dyn }}=\lambda L / D \approx 0,047$ bis 0,033 , abhängig von der Strömungsgeschwindigkeit. Für den Staudruck im Messrohr gilt in guter Näherung $p_{\text {dyn }} \approx \frac{\gamma}{2} p_{0} \mathrm{Ma}^{2}\left(1-1,6 \mathrm{Ma}^{2}\right)$.

Aufgrund des über die Resonatorkammern angebrachten Gitters ist der freie Durchmesser ca. 49,5 mm. Mit dem um 1\% kleineren Durchmesser und damit 2\% kleineren Querschnitt nimmt der Staudruck um 4\% zu. Da der Staudruck im freien Querschnitt $(D=50 \mathrm{~mm})$ zur Normierung dient, wäre für eine ausgebildete Strömung ein um 5\% höherer Wert zu erwarten. Bedeutender wird diese Korrektur bei den kleineren freien Querschnitten.

Ohne Zentralkörper mit 16 offenen Kammern sind zwei unabhängige Messungen aufgetragen (blaue Kurven mit Kreisen bzw. Quadraten). Hier kann abgeschätzt werden, 


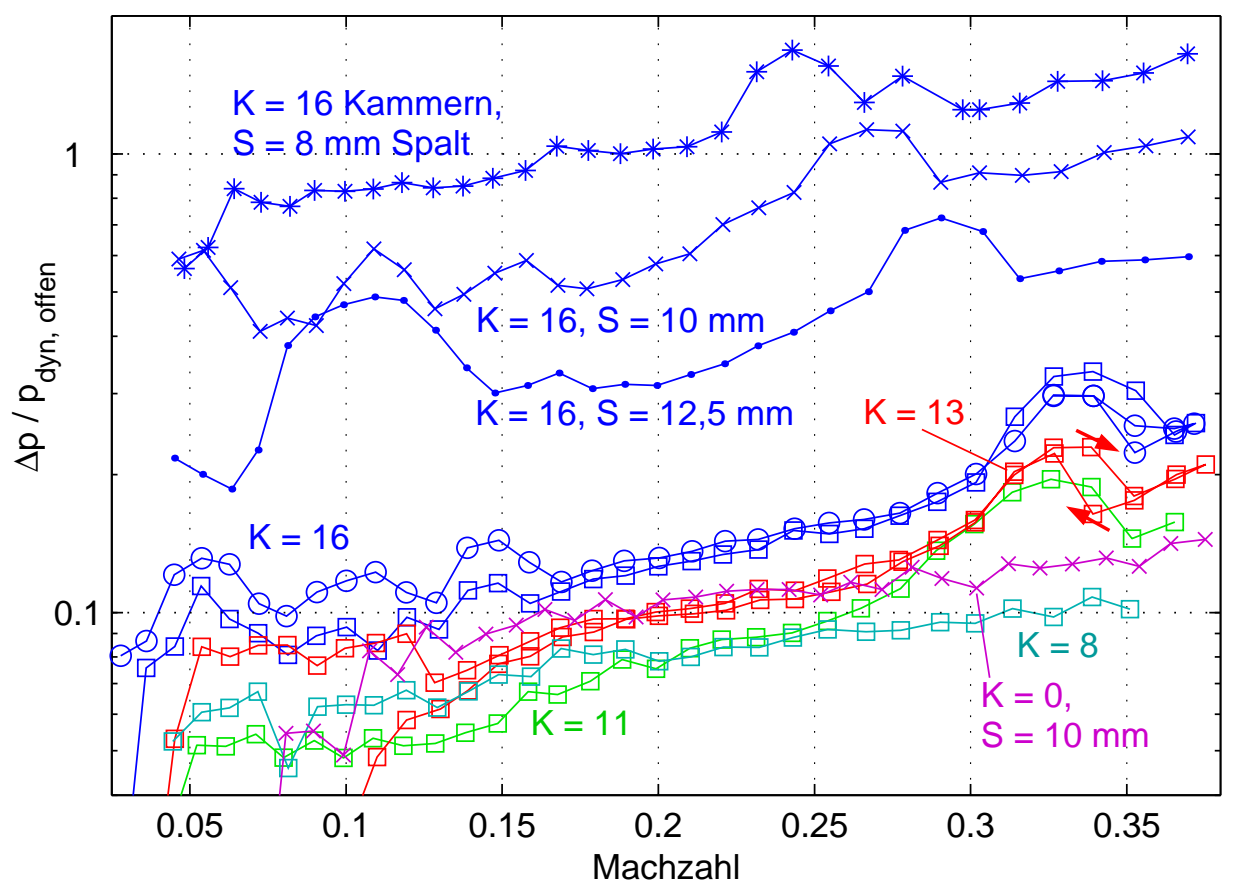

Abb. 2.21: Druckabfall am Resonatorabschnitt mit und ohne Zentralkörper als Funktion der Machzahl im freien Ringspalt für verschiedene Ringspaltbreiten $S$ und Anzahlen an offenen Resonatoren $K$. Kurven ohne Angabe einer Spaltbreite gelten für den Fall ohne Zentralkörper; der Druckabfall ist auf den Staudruck im Messrohr bezogen.

wie weit unabhängige Messungen streuen. Besonders bei kleinen Machzahlen ist aufgrund der geringen Druckdifferenzen die Messung unsicher. Insgesamt ist ein Anstieg des normierten Druckabfalls mit der Machzahl zu sehen, bei einem glatten Rohrstück würde die Rohrreibung abnehmen. Wenn die Rauigkeiten (hier etwa durch das Gitter über den Kammern) in einem harten Rohr größer als die viskose Unterschicht der Strömung sind, ist $\lambda$ unabhängig von Ma.

Je mehr Resonatorkammern abgeklebt sind, um so geringer ist der Druckabfall. Die dabei verdeckten Rauigkeiten haben also einen größeren Einfluss als die Änderung des Querschnitts, der durch die Abklebung ein wenig kleiner wird. Allerdings steigt auch bei vollständig abgeklebten Resonatorkammern der Druckabfall weiter mit der Machzahl an, hier für den Fall mit $S=10 \mathrm{~mm}$ eingezeichnet.

Um zu sehen, wieweit eine Hysterese vorhanden ist, wurde für den Aufbau mit 13 offenen Resonatorkammern die Machzahl, nachdem sie ihren Maximalwert erreicht hat, wieder auf $\mathrm{Ma}=0$ verringert. Am Punkt $\mathrm{Ma}=0,34$ tritt Hysterese auf, die in der 
Abbildung durch Pfeile gekennzeichnet ist. Bei den kleineren Machzahlen unterhalb $\mathrm{Ma}=0,13$ laufen die Kurvenabschnitte stark auseinander. Das liegt vermutlich an einer Ungenauigkeit der Druckaufnehmer, die spontan um Werte, die etwa $20 \mathrm{~Pa}$ entsprechen springen. Hier scheint es, als ob solch ein Sprung schon bei der Erhöhung (obere Kurve) von $\mathrm{Ma}=0,12$ auf 0,13 stattfand. Für eine Messung mit 16 Resonatorkammern ist ebenfalls die Hysterese um Ma $=0,35 \mathrm{zu}$ sehen, hier geht die Machzahl nur auf 0,32 zurück.

Drei weitere Messkurven sind eingezeichnet, bei denen im Resonatorabschnitt statt des offenen Rohres nur ein Ringspalt der Breite $S$ offen ist, was durch den Einbau eines Zentralkörpers erreicht wird (s. Abb. 2.3). Gezeigt ist hier wieder der Unterschied, der durch den Einbau des Resonatorabschnitts zustande kommt; die Referenzmessung wurde also mit eingebautem Zentralkörper durchgeführt. Normiert ist die Druckdifferenz mit dem Staudruck im offenen Rohr, aufgetragen über der Machzahl im Ringspalt.

Mit abnehmender freien Spaltbreite nimmt der Widerstand zu. Das war nicht zwingend zu erwarten, da hier die Differenz zu einer Vergleichsmessung mit gleichem Querschnittsprofil dargestellt ist. Der Anstieg kommt also durch die Beschaffenheit des Resonatorabschnitts zustande. Eine wahrscheinliche Ursache ist, dass die Strömung unmittelbar vor dem Resonatorabschnitt beschleunigt wird, und damit das Strömungsprofil völliger ist. Damit kommen die Effekte an der Wand stärker zu tragen. Mit vollständig abgeklebten Resonatoren (violette Kurve) wird $\Delta p / p_{\text {dyn }}$ zwar um einen Faktor von etwa fünf reduziert, liegt aber (wie alle Messungen) über der obigen Abschätzung von rund 0,04.

Ein weiterer Mechanismus, der zum Druckabfall beiträgt, ist die Instabilitätswelle. Ohne Zentralkörper wächst der Druckabfall von $\mathrm{Ma}=0,31$ bis 0,34 besonders stark, hier tritt auch das selbsterregte Pfeifen um $1,2 \mathrm{kHz}$ am stärksten auf. Für größere Machzahlen ist der Druckabfall wieder etwas geringer. Die Hysterese im Druck entspricht der in Abb. 2.7 gezeigten Hysterese im Spektrum.

Die Normierung mit dem dynamischen Druck im Rohr ohne Zentralkörper ist geeignet, um den Einfluss des Resonatorabschnitts auf eine gegebene Strömung zu beschreiben. Soll z. B. ein akustisch steuerbares Ventil gebaut werden, so ist hier die Widerstandserhöhnung zu sehen, die alleine durch den Einbau des Resonatorabschnitts kommt - bei offenem Ventil. Im Folgenden wird diese Normierung benutzt, wenn kein Zentralkörper vorhanden ist.

Zum Vergleich zwischen den verschiedenen Zentralkörpern ist eine andere Normierung besser geeignet. Diese ist in Abb. 2.22 mit den größtenteils gleichen Messreihen wie Abb. 2.21 dargestellt. Dabei wird von der gemessenen Änderung des Druckabfalls $\Delta p$ der Wert abgezogen, den ein entsprechend langes Stück hartes Rohr $\Delta p_{\text {Rohr }}$ liefert. 


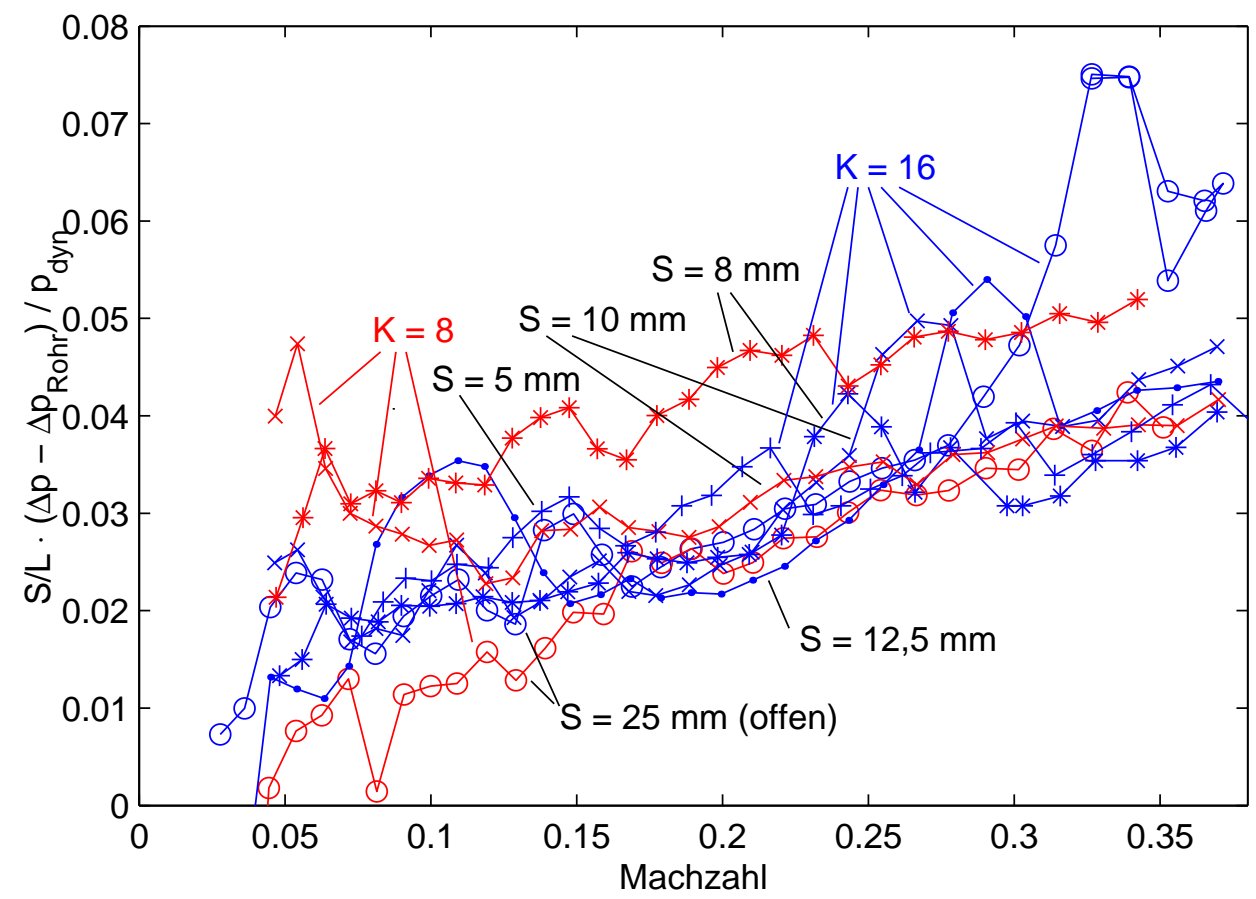

Abb. 2.22: Wie Abb. 2.21, jedoch nach Abzug des Druckabfalls, der bei Ersetzen der Resonatoren durch eine harte Rohrwand entsteht. Zudem ist der Druckabfall durch $L / S$ dividiert und auf den Staudruck im Ringspalt normiert.

Dieser Wert wird durch den Staudruck im Spalt geteilt und mit der Länge der offenen Resonatorkammern, ausgedrückt in Spaltbreiten $L / S$ normiert. Ohne Zentralkörper ist $S=25 \mathrm{~mm}$ gesetzt. Für die verschiedenen Rohrquerschnitte mit 16 und mit acht offenen Resonatorkammern ergeben sich ähnliche Kurven. Ohne Zentralkörper und mit 16 offenen Resonatorkammern unterscheiden sich die beiden Normierungen um den Faktor $L / S=3,2$, mit elf Resonatorkammern ist $L / S=2,2$.

\subsubsection{Einfluss von Schall auf den Druckabfall}

Nun soll der Einfluss aus dem Rohr stromauf eingebrachten Schalls auf den statischen Druckabfall an dem Abschnitt mit 16 offenen Resonatorkammern ohne Zentralkörper betrachtet werden. In Abb. 2.23 ist der Druckabfall als Funktion der Schallamplitude für zwei Machzahlen aufgetragen. Für beide Machzahlen sind Frequenzen ausgewählt, die sich an der Frequenz $f_{\max }$ orientieren, bei der die maximale Verstärkung auftritt. Es ist jeweils eine Kurve zu einer Frequenz unterhalb, einer nahe und einer oberhalb von $f_{\max }$ aufgetragen. Die höchste Frequenz ist bei $\mathrm{Ma}=0,25$ mit $1127 \mathrm{~Hz}$ nur relativ 


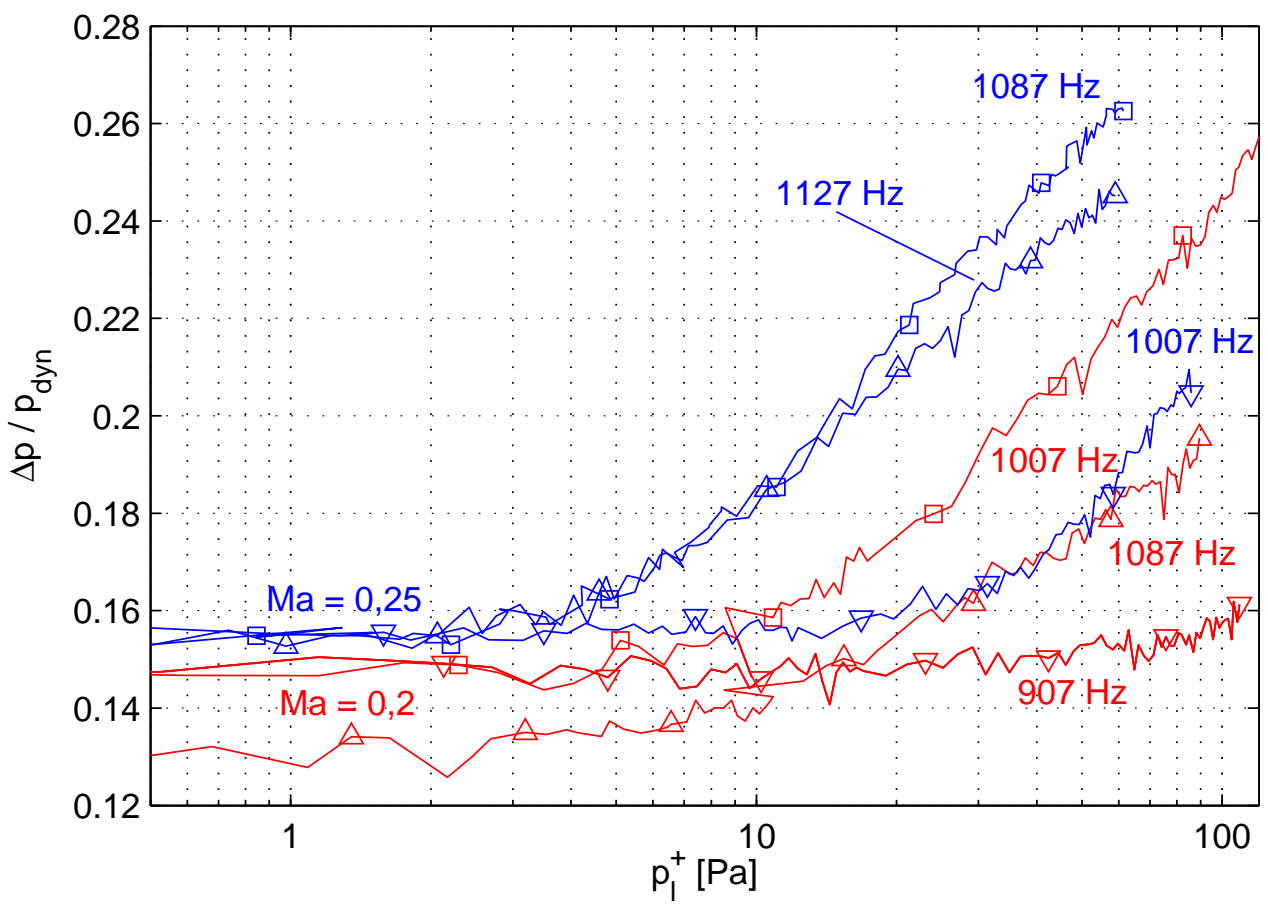

Abb. 2.23: Druckabfall $\Delta p / p_{\text {dyn }}$ am Abschnitt mit 16 offenen Resonatorkammern in Abhängigkeit von der Schallamplitude für Ma =0,2 und 0,25 (mit $p_{\text {dyn }}=23,5$ und 37,1 mbar) für Frequenzen im Bereich maximalen Transmissionsfaktors.

knapp oberhalb $f_{\max }$, da noch ein deutlicher Abstand zu dem Einbruch um $1,2 \mathrm{kHz}$ gehalten werden soll.

Wenn eine Grenzamplitude überschritten ist, steigen alle Kurven mit der Schallamplitude $p_{l}^{+}$an. Wie auch zu erwarten war, ist der Einfluss auf den Druck nahe $f_{\max }$ am größten. Bemerkenswert ist, dass $\Delta p / p_{\text {dyn }}$ auch mit der Machzahl ansteigt, da die Normierung auf $p_{\text {dyn }}$ einen mehr als quadratisch höheren absoluten Druckabfall erfordert.

Wenn eine gewisse Anregungsschwelle überschritten wird, wachsen die Kurven in der halblogarithmischen Darstellung linear an. Beispielsweise steigt bei $\mathrm{Ma}=0,25 \mathrm{mit}$ $f_{s} \approx f_{\max }$ und $p_{l}^{+}>5 \mathrm{~Pa}$ der Wert $\Delta p / p_{\text {dyn }}$ um 0,02 , wenn $p_{l}^{+}$verdoppelt wird. Die Steigungen variieren für die verschiedenen Kurven. In der Abbildung 2.10 der Amplitudenabhängigkeit der Transmissionsfaktoren war ein ähnliches Verhalten sichtbar: bis zu einer Grenzamplitude hat der Transmissionsfaktor einen konstanten Wert, darüber nimmt er in doppeltlogarithmischer Auftragung linear ab. Passt man jeweils an die beiden linearen Abschnitte Geraden an und bestimmt über deren Schnittpunkt die 
Grenzamplitude, so lässt sich jedoch kein einheitlicher Zusammenhang für die verschiedenen Machzahlen und Frequenzen erkennen.

Bei sehr kleinen Beschallamplituden muss der Druckabfall gleich dem ohne Schalleinfluss sein. Für Ma $=0,2$ geht der Druckabfall für kleine $p_{l}^{+}$jedoch nicht auf den gleichen Wert für alle drei Frequenzen, was auf eine Ungenauigkeit in der Druckmessung zurückzuführen ist.

Im Folgenden wird der schallinduzierte Druckabfall $\Delta p_{\text {ak }}$ gezeigt, der als Differenz des Druckabfalls mit und ohne Beschallung (mit der halben Maximalamplitude) definiert ist, $\Delta p_{\mathrm{ak}}=\Delta p\left(A_{s}=0,5 A_{s}^{\max }\right)-\Delta p\left(A_{s}=0\right)$. Abb. 2.24 stellt den schallinduzierten Druckabfall $\Delta p_{\text {ak }}$ normiert auf $p_{\text {dyn }}$ und auf die Wandlänge, gemessen in Spaltbreiten $\frac{L}{S}$ in Abhängigkeit von der Schallfrequenz dar. Für 16 Kammern $^{7}$ ist $L=16 B=80 \mathrm{~mm}$, die Kurven ohne Angabe von $S$ sind bei freiem Rohrquerschnitt aufgenommen, hier ist $S=25 \mathrm{~mm}$ gesetzt. Die Messung wurde mit den Lautsprechern an der ersten Resonatorkammer durchgeführt. Gezeigt sind Messungen von $\mathrm{Ma}=0,1$ bis 0,3 . Der maximale Einfluss auf den Druck wird in der Nähe von Frequenzen erzielt, bei denen auch im Spektrum das Maximum liegt.

In dieser Normierung wird der maximale schallinduzierte Druckabfall bei Ma $=0,25$ erzielt. Er ist etwas größer als der Druckabfall am Resonatorabschnitt ohne Schall. Durch den Schall kann also die Rohrreibung in etwa verdoppelt werden. Bei Beschallung aus dem Rohr wird dieser Wert nicht ganz erreicht.

Die Abbildung 2.25 zeigt einen Vergleich des Druckabfalls bei Ma $=0,25$ mit 16 bzw. elf Resonatorkammern ${ }^{8}$. Gestrichelt ist der Schalldruck der jeweiligen Frequenz gezeichnet. Der maximale Effekt auf den Druck ist bei elf Resonatoren fast nur halb so groß wie bei 16, zu tieferen Frequenzen eher noch kleiner. Bei höheren Frequenzen nähern sich dagegen die Kurven für beide Resonatorenzahlen. Der Einfluss auf den Druck wird dabei allerdings geringer.

Die Kurven für den Schalldruck haben ihr Maximum bei etwas tieferen Frequenzen als der statische Druckabfall. Der Unterschied in den Frequenzen scheint systematisch zu sein, er tritt auch bei allen in Abb. 2.24 aufgetragenen Messungen auf (nicht eingezeichnet). Das Maximum des Schalldrucks ist im Vergleich zu $\Delta p_{\text {ak }}$ bei elf Kammern höher als bei 16 Kammern. Es geht zunehmender Kammerzahl ein geringerer Bruchteil der Energie, die aus dem Druckabfall stammt, in den abgestrahlten Schall.

\footnotetext{
${ }^{7}$ Die Breite der Trennbleche sowie die Länge der Halterung gehen nicht ein.

${ }^{8}$ Die Abweichung zur Auftragung 2.24 kommt daher, dass ein anderer Referenzlautsprecher verwendet wurde.
} 


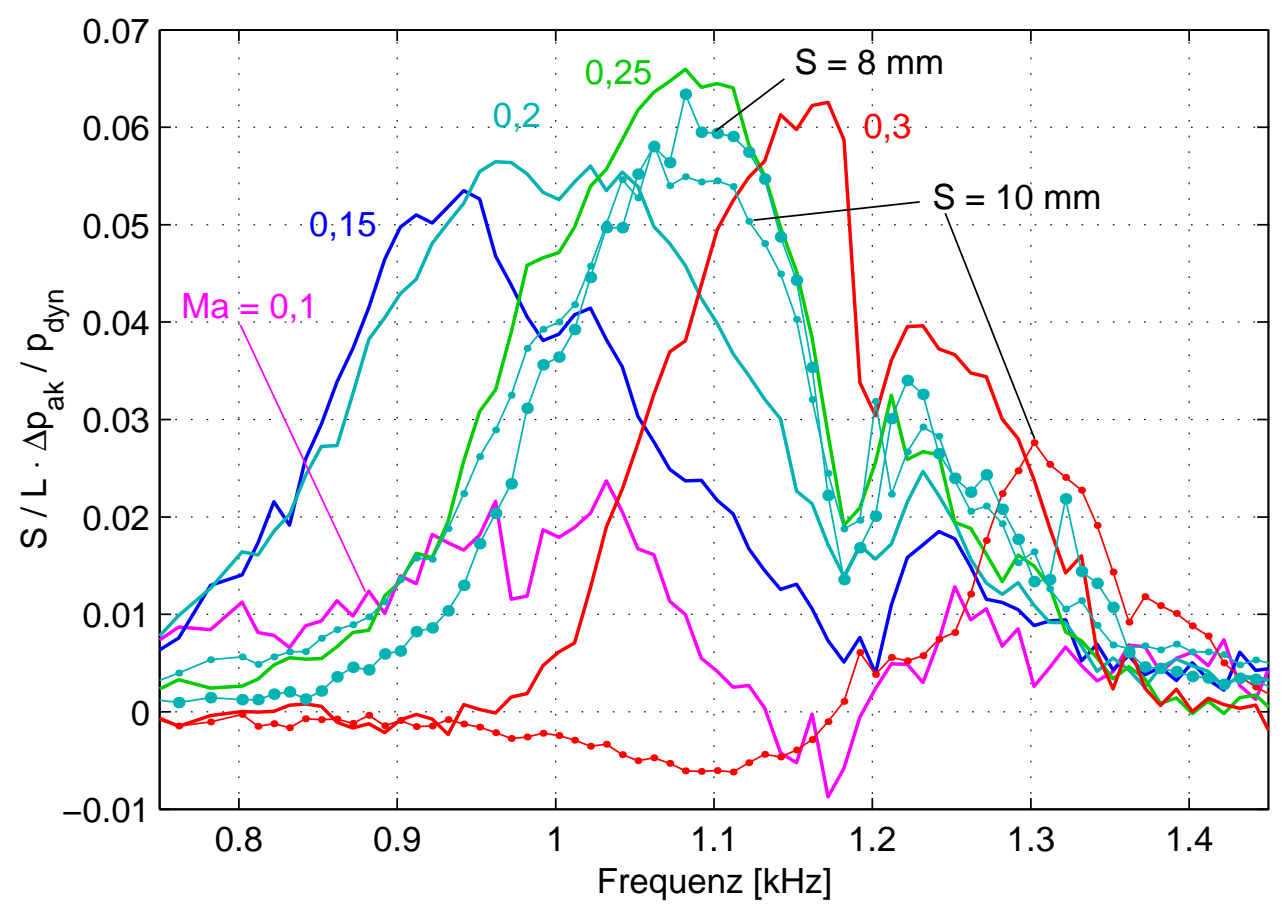

Abb. 2.24: Schallinduzierte Änderung des Druckabfalls $\frac{S}{L} \Delta p_{\text {ak }} / p_{\text {dyn }}$ mit und ohne Zentralkörper am Abschnitt mit 16 Resonatoren als Funktion der Frequenz. Der Schall wird mit Lautsprechern an der linken Resonatorkammer erzeugt. Kurven ohne Angabe von $S$ gelten für den Fall ohne Zentralkörper.

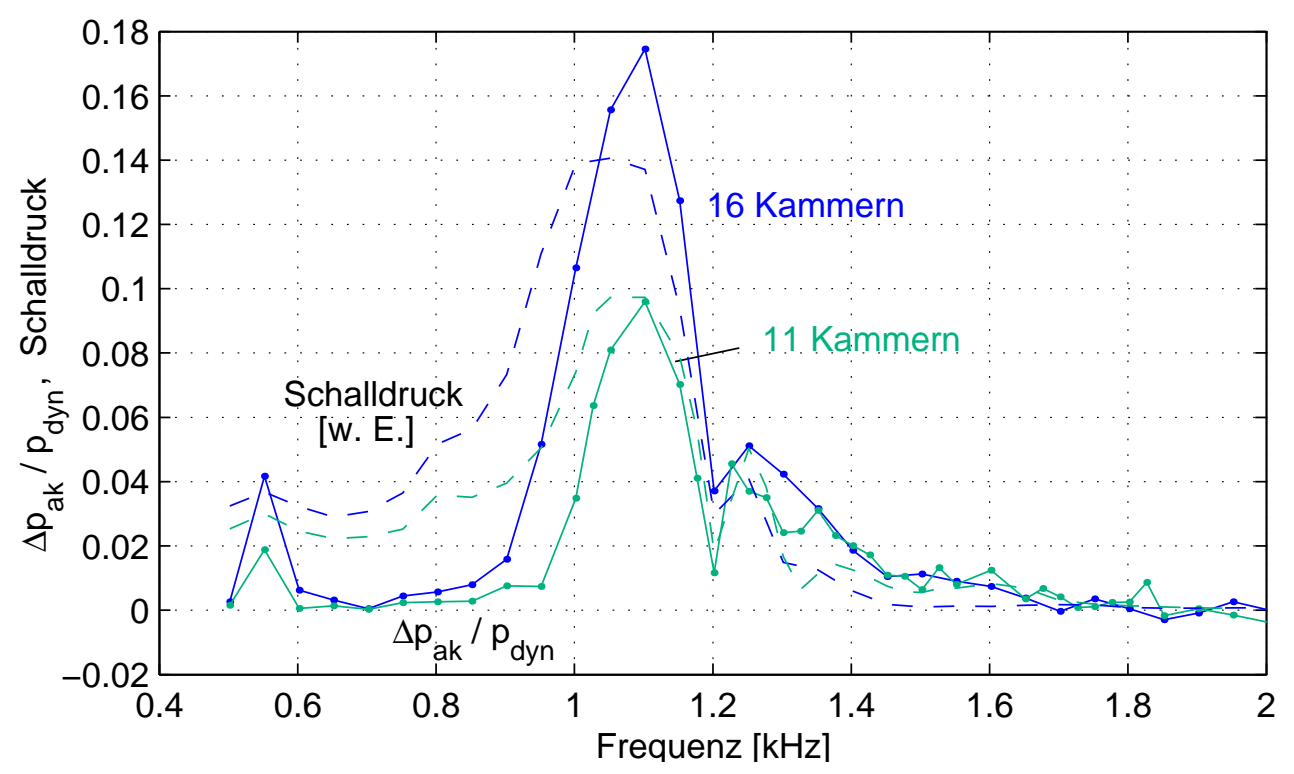

Abb. 2.25: Schallinduzierter Druckabfall $\Delta p_{\mathrm{ak}} / p_{\text {dyn }}$ am Abschnitt mit elf bzw. 16 Resonatoren ohne Zentralkörper in Abhängigkeit von der Frequenz für $\mathrm{Ma}=0,25$ ( $p_{\text {dyn }}=3,69$ mbar $)$, Beschallung aus der ersten Resonatorkammer. Zum Vergleich ist der stromab abgestrahlten Schalldruck (willkürliche Einheiten) gestrichelt dargestellt. 


\subsection{Höhere akustische Moden}

Soll der durch den Schall verursachte Druckabfall technisch genutzt werden, beispielsweise um einen tieffrequenten Lautsprecher zu erstellen (siehe Lange [15]), so dient in der Amplitude modulierter Schall zur Erzeugung tieffrequenter Druckschwankungen. Der mit dem Lautsprecher erzeugte Schall dient nur der Steuerung und ist ansonsten unerwünscht. Lange hat ihn stromab mit einem gedämmten Resonator beseitigt. Wird im Resonator eine höhere Schallmode angeregt, die sich im hartwandigen Rohr nicht ausbreiten kann, so ist eine Dämmung unnötig, die mit zusätzlichem Druckverlust einhergeht. Im Folgenden werden Ergebnisse zur Anregung mit einer solchen höheren Mode $\sim \exp (i m \phi)$ gezeigt. Gezielt können hier nur zirkulare Moden angeregt werden $m= \pm 1$.

\subsubsection{Druckschwankungen in der letzten Resonatorkammer}

Wie schon bei der ebenen Mode soll zunächst das Spektrum der Druckschwankungen betrachtet werden, die durch die turbulente Strömung angeregt werden. Da die höheren Moden im hartwandigen Rohr nicht ausbreitungsfähig sind, wird statt des Leistungsspektrums im Rohr die Phase zwischen zwei Mikrofonen in der Resonatorkammer stromab gemessen. Sie ist in Abb. 2.26 für zwei unterschiedliche Winkel $\alpha$ zwischen den Mikrofonen in der letzten von 16 Resonatorkammern dargestellt; oben ist $\alpha=120^{\circ}$, unten $180^{\circ}$. Die Phase wird durch die Kohärenzfunktion

$$
\gamma_{W_{1} W_{2}}(f):=\frac{\mathcal{F}^{*}\left\{w_{1}\right\} \cdot \mathcal{F}\left\{w_{2}\right\}}{\left|\mathcal{F}\left\{w_{1}\right\}\right|\left|\mathcal{F}\left\{w_{2}\right\}\right|}
$$

der Mikrofonsignale $w_{1}(t)$ und $w_{2}(t)$ bestimmt. Ist der Betrag der Kohärenz kleiner als $1 / 3$, so sind die Werte blass gezeichnet. Gleichphasige Bereiche sind grün, gegenphasige rot dargestellt. Eine Farbtabelle ist im Symbolverzeichnis (Abb. 0.2) zu finden.

In einem nicht durchströmten Rohr können die ausbreitungsfähigen Moden berechnet werden. Mit wachsender Frequenz nimmt die Zahl der Moden zu; Abb. 2.27 stellt zum Vergleich mit Abb. 2.26 schematisch die tieffrequenten Moden dar. Für den Radius der Resonatorkammern $R_{a}=100 \mathrm{~mm}$ ist die Grenzfrequenzen angegeben, ab der die jeweilige Mode ohne Strömung ausbreitungsfähig ist.

Im Vergleich mit der Messung können Abweichungen auftreten, da das Rohr durchströmt ist und sich so die Randbedingung zwischen Rohr und Resonator ändert. Zudem können evtl. zusätzlich hydrodynamische Moden auftreten. 


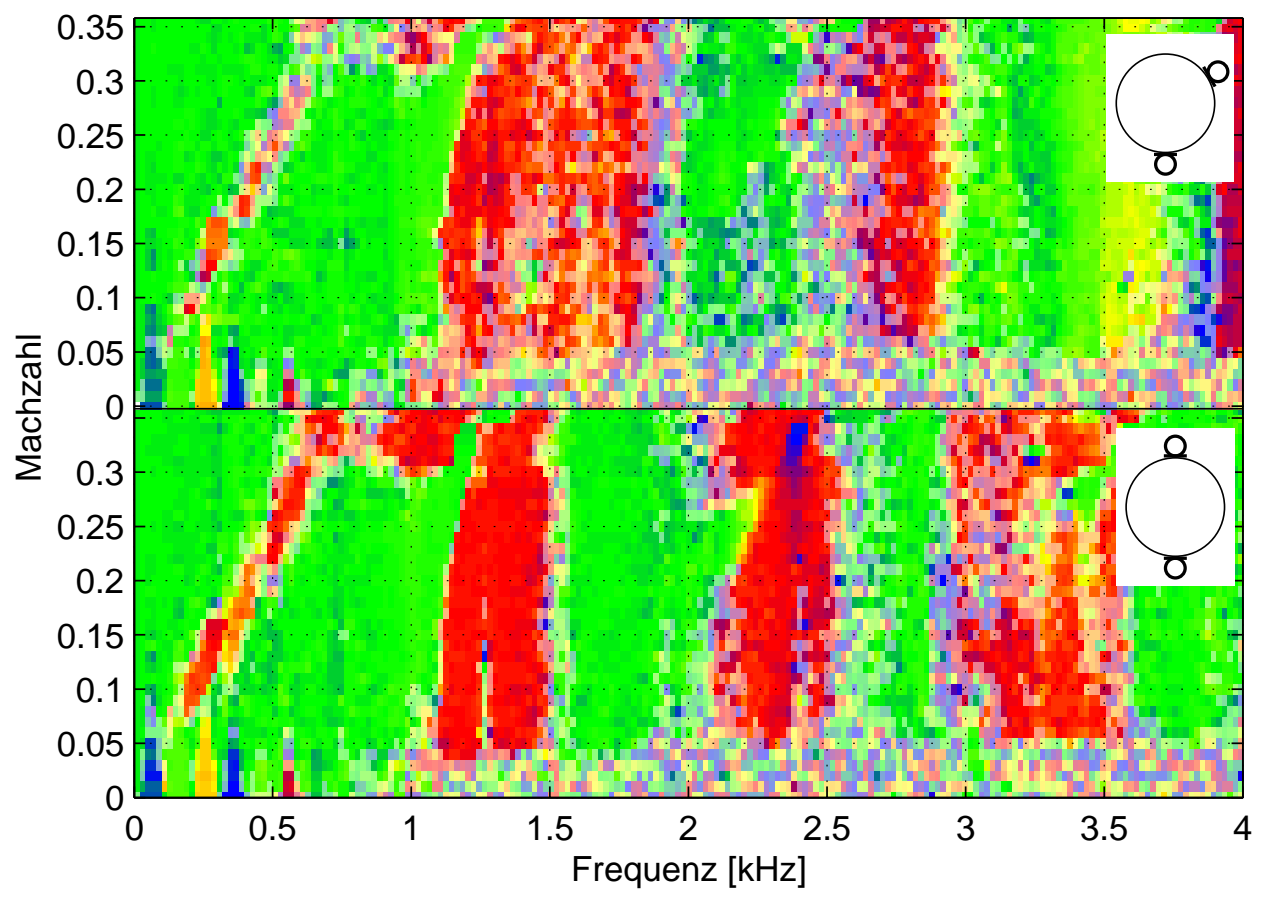

Abb. 2.26: Phase des Kreuzspektrums zweier Mikrofonsignale in der stromabseitigen Resonatorkammer in Abhängigkeit von der Machzahl. Oben beträgt der Winkel zwischen den Mikrofonen $120^{\circ}$, unten $180^{\circ}$. Farbcodierung siehe Abb. $0.2 \mathrm{im}$ Symbolverzeichnis.
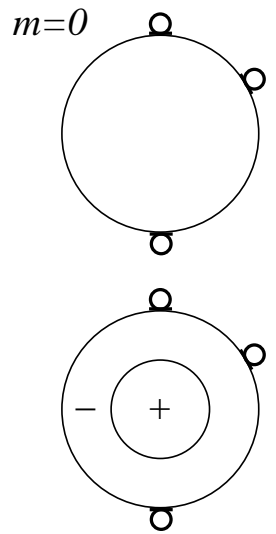

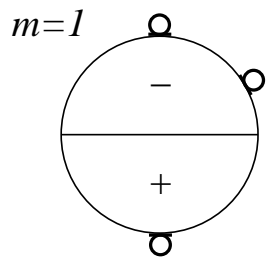

ab $1005 \mathrm{~Hz}$

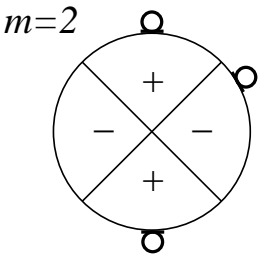

$1665 \mathrm{~Hz}$

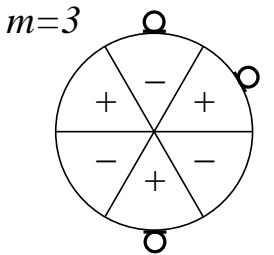

$2293 \mathrm{~Hz}$

$2092 \mathrm{~Hz}$ ausbreitungsfähig

Abb. 2.27: Schematsiche Darstellung der Moden $[\exp (+i m \phi)+\exp (-i m \phi)]$ mit Positionen von Messmikrofonen sowie der ersten höheren radialen Mode. Es sind die Frequenzen angegeben, oberhalb der die jeweiligen Moden in einem Rohr mit Radius $100 \mathrm{~mm}$ und $c=343 \mathrm{~m} / \mathrm{s}$ ausbreitungsfähig ist. 
In der Messung sind zu beiden Winkeln $\alpha$ für Frequenzen bis 1,5 kHz die Phasen in beiden Auftragungen gleich. Nur die gegenphasigen Bereiche sind bei $\alpha=120^{\circ}$ schwächer ausgeprägt. Da im Rohr keine der Winkelrichtungen $\exp ( \pm i \phi)$ ausgezeichnet ist und daher auch eine gegenphasige Mode zeitweise an den Mikrofonen ein gleichphasiges Signal liefern kann, sollte hier $\gamma_{W_{1} W_{2}} \approx-1 / 3$ sein. Tiefe Frequenzen sind bis $1,1 \mathrm{kHz}$ für Machzahlen bis 0,3 gleichphasig, nur unterbrochen durch einen Streifen, dessen Mittenfrequenz linear mit der Strömungsgeschwindigkeit entsprechend 2,2 kHz·Ma anwächst. Für Ma $>$ 0,3 ist die Kohärenz bei Frequenzen oberhalb dieses Streifens gegenphasig, nur unterbrochen durch den gleichphasigen Anteil um $1,2 \mathrm{kHz}$, bei dem die selbsterregte Schwingung auftritt. Zwischen $1,1 \mathrm{kHz}$ und $1,5 \mathrm{kHz}$ sind die Signale ebenfalls gegenphasig. Auch dieser Bereich ist wieder durch einen sehr schmalen Streifen für kleine Machzahlen bei $1,26 \mathrm{kHz}$ geteilt, der nur in der weniger verrauschten Auftragung für $\alpha=180^{\circ} \mathrm{zu}$ erkennen ist. Oberhalb von $\mathrm{Ma}=0,2$ ist er nicht mehr $\mathrm{zu}$ sehen.

Für Frequenzen über $1,5 \mathrm{kHz}$ liefern die Mikrofone unterschiedliche Phasen, die bis $1,8 \mathrm{kHz}$ der Mode $\sim \exp ( \pm 2 i \phi)$ entsprechen. Noch höhere Frequenzen können nicht mehr eindeutig einer Mode zugeordnet werden; die erste radiale Mode müsste bei $2,1 \mathrm{kHz}$ einsetzen. Sie kann mit den Mikrofonen, die den gleichen Abstand von der Rohrachse haben, nicht aufgelöst werden. Die Mikrofone liefern gleichphasige Werte, wenn diese Mode dominant ist.

Die Phase an den Mikrofonen ist bis auf die beiden beschriebenen Ausnahmen im gesamten Frequenzbereich nahezu unabhängig von der Machzahl. Für fast alle Frequenzen sind die Mikrofone entweder gleich- oder gegenphasig. Das ist plausibel, da im Kanal keine Richtung ausgezeichnet ist und daher beide Anteile $\pm m$ im Mittel gleich häufig vorkommen sollten.

Aus der Phase kann nicht direkt auf die Ausbreitungsfähigkeit einer Mode geschlossen werden. So ist es denkbar, dass die betrachtete Instabilitätswelle lokal angeregt wird und sich sowohl durch eine ebene als auch durch eine zirkulare Störung anregen lässt.

\subsubsection{Einfluss externer Beschallung}

In Abb. 2.28 werden die beiden akustisch angeregten Moden $m= \pm 1$ und $m=$ 0 mit der selbsterregten Schwingung verglichen. Die durchgezogenen Linien stellen die Phase der Kohärenz zweier Signale dar, die mit gegenüberliegenden Mikrofonen $\left(180^{\circ}\right)$ in der letzten Resonatorkammer aufgenommen wurden, im oberen Teilbild für $\mathrm{Ma}=0,32$ und im mittleren für $\mathrm{Ma}=0,25$. Unten ist der Betrag von $\gamma_{W_{1} W_{2}}$ für die beiden Machzahlen aufgetragen. Die Symbole in den beiden oberen Darstellungen 


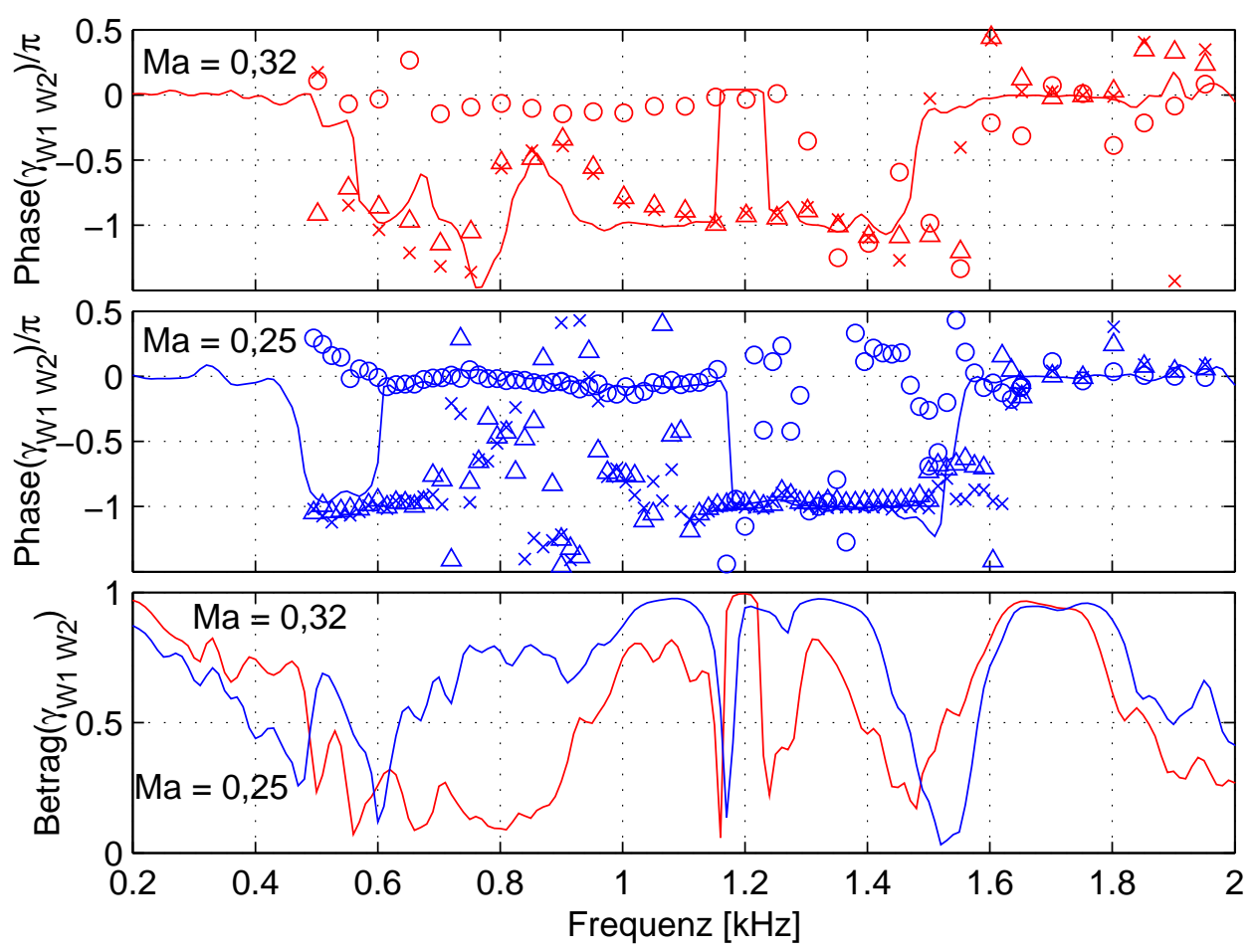

Abb. 2.28: Phase und Betrag der Kohärenz $\gamma_{W_{1} W_{2}}$ von Signalen gegenüber liegender Mikrofone in der letzten der 16 Resonatorkammern bei Ma =0,32 (rot) und $\mathrm{Ma}=0,25$ (blau). Linien: ohne externen Schalleintrag, Symbole: mit Beschallung aus der ersten Resonatorkammer. Kreise: gleichphasige $\left(\phi_{L}=0^{\circ}\right)$, Kreuze: gegenphasige $\left(\phi_{L}=180^{\circ}\right)$, Dreiecke: $\phi_{L}=120^{\circ}$ phasenverschobene Lautsprecheranregung.

geben die Phasendifferenz bei Beschallung mit verschiedenen Frequenzen wieder 9 . Für diese Messung sind drei Lautsprecher über den Umfang der ersten Resonatorkammer angebracht (s. Abb. 2.2).

Da auch die Lautsprecher gleichen Typs nicht exakt gleich arbeiten, werden sie für jede Frequenz neu eingestellt. Dazu wird zunächst ein Lautsprecher mit fester Amplitude und Phase betrieben, und der zweite gegenphasig eingestellt $\left(\phi_{L}=180^{\circ}\right)$, so dass in den Messrohren (stromauf und stromab des Resonatorabschnitts) ein möglichst wenig zur verwendeten Frequenz kohärentes Signal ankommt. Die Phase der Signale in der Resonatorkammer nach dieser Adaption ist mit Kreuzen in Abb. 2.28 eingtragen. Dann wird der so adaptierte Lautsprecher in seiner Phase um $60^{\circ}$ gedreht und

${ }^{9}$ Bei Beschallung hängt die Kohärenzfunktion von der Schallfrequenz ab, und ihr Betrag liefert in dem hier betrachteten Zusammenhang keine zusätzliche Information. 
der dritte Lautsprecher ebenso adaptiert. Damit ist die Phasendifferenz zwischen je zwei Lautsprechern $\phi_{L}=120^{\circ}$. Diese Messungen sind mit Dreiecken gekennzeichnet. Schließlich wird die Phase des zweiten Lautsprechers um $120^{\circ}$ und die des dritten um $240^{\circ}$ gedreht, so dass die drei Lautsprecher gleichphasig betrieben werden, $\phi_{L}=0^{\circ}$. Die zugehörigen Ergebnisse sind mit Kreisen in die Darstellung eingetragen.

Bei tiefen Frequenzen sowie bei Frequenzen nahe der Resonanz ist die Amplitude der Lautsprecher sehr groß. Es ist daher möglich, dass sie dann im nichtlinearen Bereich betrieben werden. Wenn die Phase der Anregung gedreht wird, ändert sich die Impedanz, gegen die die Lautsprecher betrieben werden. Damit ändert sich auch das Abstrahlverhalten. Wenn der dritte Lautsprecher hinzugenommen wird, stört dies nicht (solange nur Moden mit $m \leq 1$ ausbreitungsfähig sind), da dieser wieder so eingestellt wird, dass die Schallwelle schließlich kompensiert ist. Bei dem gleichphasigen Betrieb ist es wahrscheinlich, dass sich die Lautsprecher gegenseitig beeinflussen. Hier könnte noch eine relativ große Komponente der gegenphasigen Mode angeregt werden.

Die Einstellung der Lautsprecher erfolgt diskret in 256 Schritten für die Phase und in 256 Amplitudenschritten. Die Phase kann somit bei einer unverrauschten Messung theoretisch im schlechtesten Fall auf $2 \pi / 512 \approx 1,2 \%$ genau eingestellt werden. Die Amplitude wird um den halben Wert der Vollaussteuerung, also um 128 variiert, was einem Diskretisierungsfehler von 0,4\% entspräche. Bei einem hier teilweise vorkommenden ungünstigen Amplitudenverhältnis kann der Wert aber auf etwa 10\% steigen. In jedem Fall wird nie eine reine Mode angeregt, sondern immer ein Modengemisch.

Ein Vergleich mit Abb. 2.27 zeigt, dass bei zwei Lautsprechern ab $1665 \mathrm{~Hz}$, bei drei Lautsprechern spätestens ab $2293 \mathrm{~Hz}$ nicht mehr eindeutig ist, welche Mode angeregt wird. Die Frequenzen können sich durch die Strömung leicht verschieben.

Zwischen den beiden Machzahlen 0,25 und 0,32, nämlich bei $\mathrm{Ma}=0,3$, die ändert sich die Phase der Kohärenz zwischen $0,7 \mathrm{kHz}$ und 1,2 kHz deutlich (siehe Abb. 2.26). So sieht man, bei welchen Frequenzen sich die Phase der turbulent erzeugten Druckschwankungen durch Schalleintrag ändern lässt.

In der oberen Darstellung von Abb. 2.28 für $\mathrm{Ma}=0,32$ liegt die Phase ohne Anregung zwischen $0,55 \mathrm{kHz}$ und $1,5 \mathrm{kHz}$ vorwiegend bei $180^{\circ}$, ausgenommen die Instabilitätswelle um $1,2 \mathrm{kHz}$, bei der nahezu die gesamte Signalenergie liegt. Bis 1,25kHz kann bei gleichphasigem Betrieb der Lautsprecher noch die ebene Mode angeregt werden. Zwischen $1,3 \mathrm{kHz}$ und $1,55 \mathrm{kHz}$ gelingt dies nicht mehr, die Phase der Mikrofone streut. $\mathrm{Ab} 1,6 \mathrm{kHz}$ sind die Mikrofonsignale wieder gleichphasig. Hier kann es sich aber auch um die Mode zu $m= \pm 2$ handeln, zumal auch bei der Anregung der Mode $m=1 \mathrm{im}$ Resonator stromab gleichphasige Signale ankommen. Von $0,5 \mathrm{kHz}$ bis $1,6 \mathrm{kHz}$ kann die 
Mode $m=1$ angeregt werden. Um $800 \mathrm{~Hz}$ scheint ein resonanzartiger Effekt vorzuliegen, hier dreht sich die Phase sowohl mit gegenphasiger als auch ohne Anregung um $180^{\circ}$. Der Betrag der Kohärenz ist jedoch klein.

Im Spektrum (nicht gezeigt) bleibt fast immer die Instabilität um 1,2 kHz dominant. Eine Ausnahme tritt bei der Anregung der Mode $m=1$ mit drei Lautsprechern bei $1,4 \mathrm{kHz}$ auf, hier kann sie vollständig unterdrückt werden. Mit zwei Lautsprechern sowie bei Nachbarfrequenzen kann noch Einfluss auf die Instabilität genommen werden, aber sie bleibt die größte Spektrallinie. Eine weitere Ausnahme ist, dass sich bei gleichphasiger Ansteuerung mit $1,25 \mathrm{kHz}$ die Instabilität auf diese Frequenz verschieben lässt.

Ein etwas anderes Verhalten tritt für $\mathrm{Ma}=0,25$ auf. Ohne Lautsprechersignal liegen für $\gamma_{W_{1} W_{2}}$ gegenphasige Werte um $0,55 \mathrm{kHz}$ und zwischen $1,2 \mathrm{kHz}$ und $1,55 \mathrm{kHz}$ vor, ansonsten ist die Kohärenz gleichphasig. Um $0,55 \mathrm{kHz}$ lässt sich auch die ebene Mode anregen. Ab 1,2 kHz gelingt das nicht mehr, bis etwa $1,4 \mathrm{kHz}$, wo die gleichphasigen Signale nicht mehr von der Mode $m= \pm 2$ unterschieden werden können. Die höhere Mode kann zwischen $0,7 \mathrm{kHz}$ und $1,1 \mathrm{kHz}$ sowie oberhalb von $1,6 \mathrm{kHz}$ nicht angeregt werden.

Leider besteht bei dem Messobjekt mit den Lautsprechern an der Resonatorkammer keine Möglichkeit, die Stärke der Anregung zu bestimmen. Die Instabilität einer höheren Mode kann daher akustisch nicht von einer ausbreitungsfähigen, nicht verstärkten Mode unterschieden werden.

Indirekt, nämlich über den statischen Druckabfall, kann aber auf Instabilitätswellen geschlossen werden: Wenn keine Instabilität angeregt wird, findet kein Energietransfer aus der Strömung statt, der statische Druck bleibt dann vom Schall unbeeinflusst.

\subsubsection{Einfluss der ersten höheren Mode auf den Druck}

Der Einfluss von Schall der Mode $m=1$ auf den Druck im Frequenzbereich von $0,2 \mathrm{kHz}$ bis $1,45 \mathrm{kHz}$ ist in Abb. 2.29 für verschiedene Machzahlen gezeigt. Für Ma = 0,2 sind zudem Messungen mit Zentralkörper bei Spaltbreiten von $10 \mathrm{~mm}$ und $8 \mathrm{~mm}$ eingezeichnet. Meist sind zwei Maxima an den Stellen vorhanden, an denen schon die Kohärenz aus Abb. 2.26 gegenphasig war. Das erste Maximum liegt bei tiefen Frequenzen und verschiebt sich proportional zur Strömungsgeschwindigkeit zu höheren. Für relativ kleine Machzahlen wird hier ein großer Druckeinfluss erzielt. Bei größeren Machzahlen dagegen tritt das zweite Maximum um 1,2 kHz stärker in Erscheinung, zu dem ein Nebenmaximum um 1,3 kHz gehört. Diese Maxima sind durch einen kleinen Einbruch 


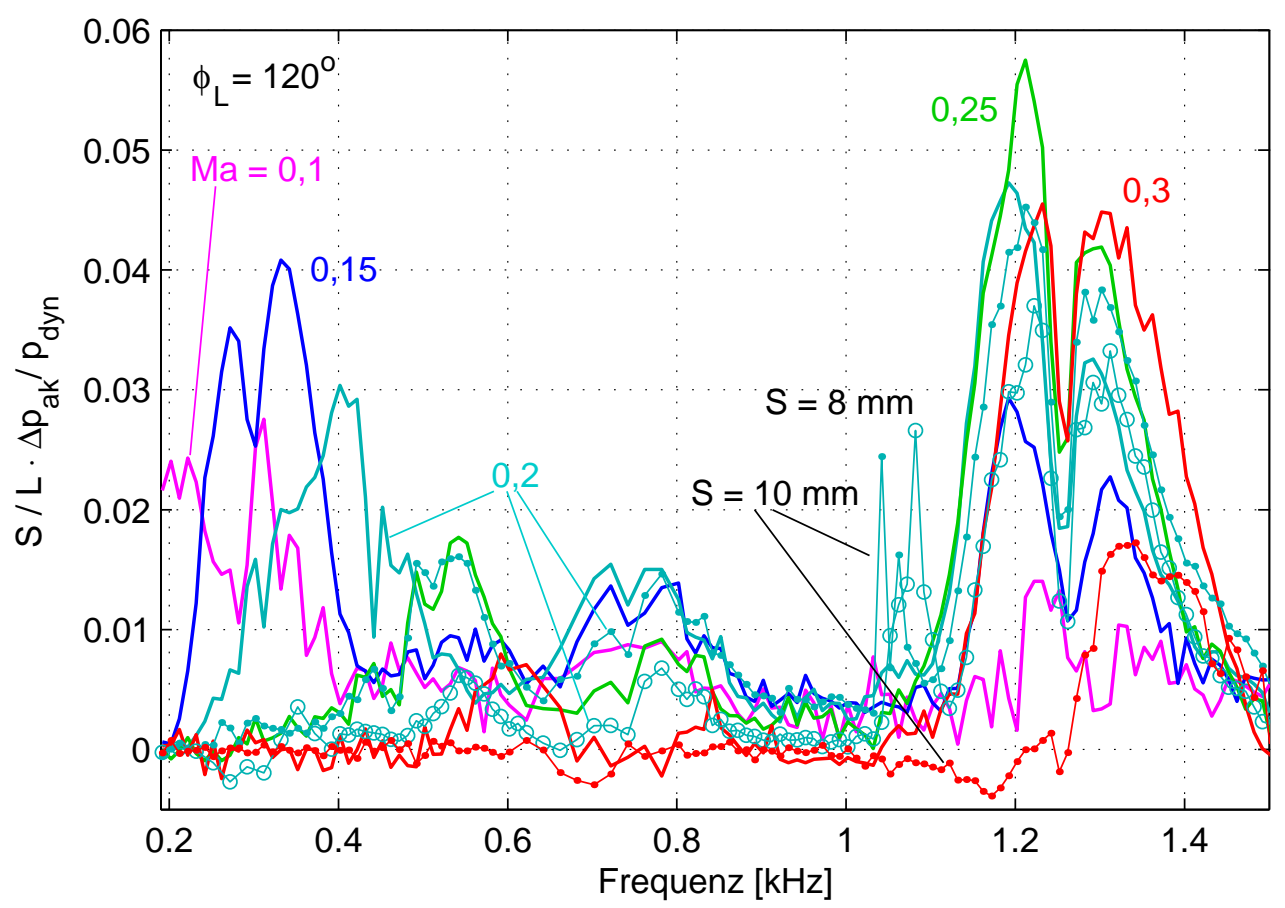

Abb. 2.29: Druckabfall $\frac{S}{L} \Delta p_{\text {ak }} / p_{\text {dyn }}$ am Abschnitt mit 16 Resonatorkammern mit (durch $S$ gekennzeichnet) und ohne ( $S$ nicht angegeben) Zentralkörper in Abhängigkeit von der Frequenz. Die Lautsprecher werden mit der Phasendifferenz $\phi_{L}=120^{\circ}$ betrieben.

getrennt. Die Lage der Maxima ist unabhängig von der Machzahl. Der Einbruch ist in der Phasenauftragung nur für kleine Machzahlen zu sehen, dort wird bei 1,26 kHz die Kohärenz klein (s. Abb. 2.26 unten).

Auf den Fall mit $S=10 \mathrm{~mm}$ bei $\mathrm{Ma}=0,3$ soll noch hingewiesen werden. Von den ausgewählten Messungen ist hier die selbsterregte Instabilität am stärksten ausgeprägt. Bei Frequenzen etwas unterhalb 1,2 kHz kann diese Instabilität durch die Anregung der höheren Mode abgeschwächt werden, so dass der Druckabfall verringert wird. Durch die Anregung der ebenen Mode bei einer benachbarten Frequenz kann die selbsterregten Instabilität stark abgeschwächt werden. Die künstlich angeregte Frequenz wird weniger verstärkt, dadurch wird der Gleichströmung insgesamt weniger Energie entzogen.

Der Einfluss auf den Druck für Frequenzen im Bereich zwischen 500 und $1650 \mathrm{~Hz}$ bei $\mathrm{Ma}=0,25$ ist in Abb. 2.30 für zwei verschieden lange Resonatorabschnitte gezeigt. Mit durchgezogenen Linien ist der durch den Schall verursachte Druckabfall über die 


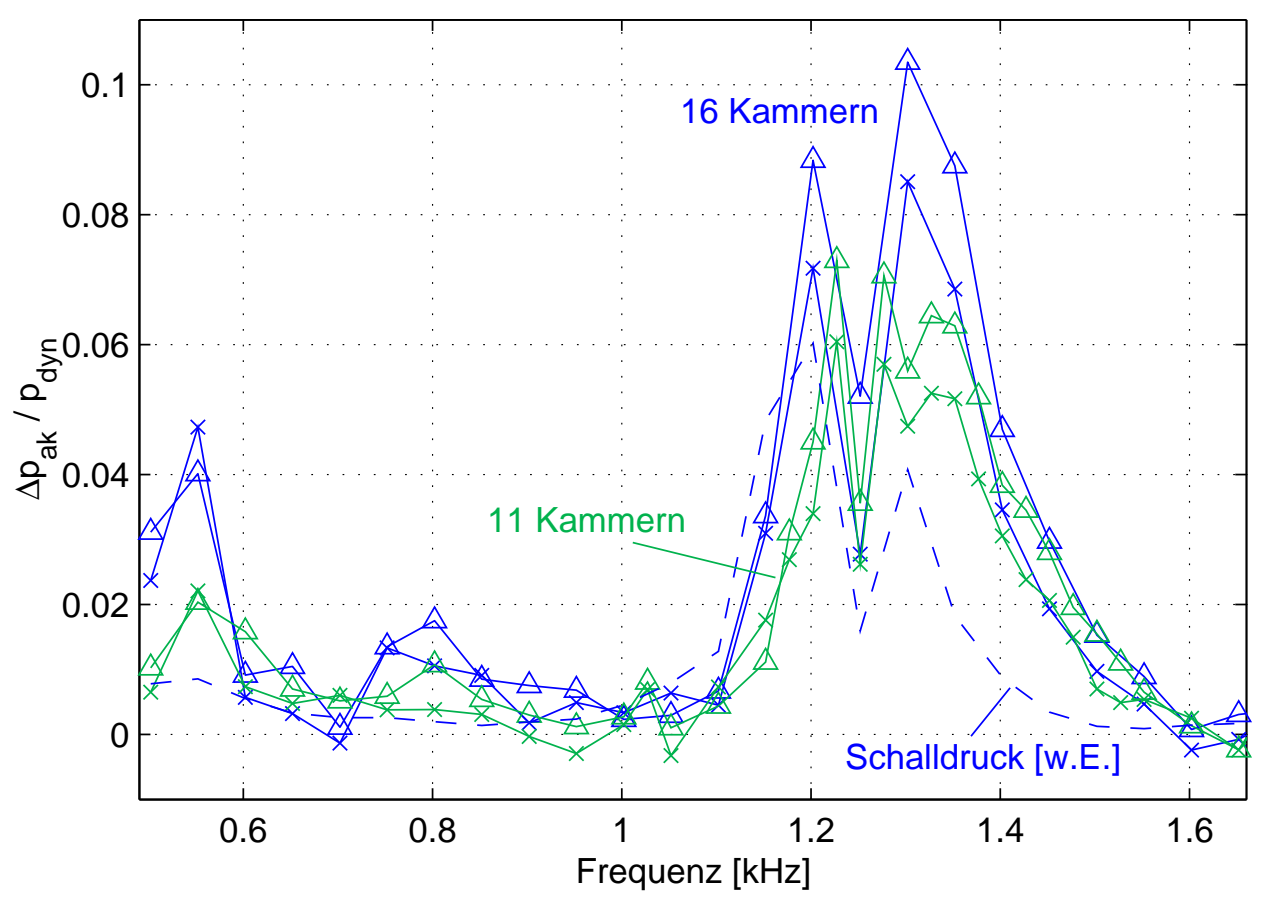

Abb. 2.30: Druckabfall $\Delta p_{\text {ak }} / p_{\text {dyn }}$ bei elf bzw. 16 Resonatoren in Abhängigkeit von der Frequenz für $\mathrm{Ma}=0,25$ bei Beschallung mit zwei gegenphasigen $\left(\times, \phi_{L}=180^{\circ}\right)$, sowie mit drei um $\phi_{L}=120^{\circ}$ phasenverschobenen Lautsprechern $(\triangle)$. Zum Vergleich ist der Schalldruck (willkürliche Einheiten) in der letzten Resonatorkammer gestrichelt eingezeichnet (nur für 16 Resonatoren).

16 (blau) 10 bzw. elf (grün) Resonatorkammern aufgetragen. Die Messergebnisse der Anregung mit zwei Lautsprechern $\left(\phi_{L}=180^{\circ}\right)$ sind mit Kreuzen, für drei Lautsprecher $\left(\phi_{L}=120^{\circ}\right)$ mit Dreiecken markiert. Für jede Frequenz wurde eine Vergleichsmessung ohne Schall durchgeführt.

Die beiden Anregungen erzielen den maximalen Druckabfall bei 1,3 kHz bzw. 1,2 kHz. Hier liegt der Druckabfall für den längeren Abschnitt etwa um das Längenverhältnis 16/11 über dem des kürzeren Abschnitts. Werden nur zwei Lautsprecher benutzt, so ist der Druckabfall jeweils um $20 \%$ geringer.

Ein kleineres lokales Maximum liegt bei $550 \mathrm{~Hz}$. Hier liegt etwa ein Faktor zwei zwischen dem Druckabfall, der mit den beiden Anordnungen erreicht wird. Ansonsten ist kein signifikanter Unterschied zwischen der Anregung mit zwei und mit drei Lautsprechern zu erkennen.

10 Eine Abweichung zur vorherigen Auftragung tritt auf, da ein anderer Referenzlautsprecher verwendet wurde, siehe auch Abb. A.2 im Anhang. 


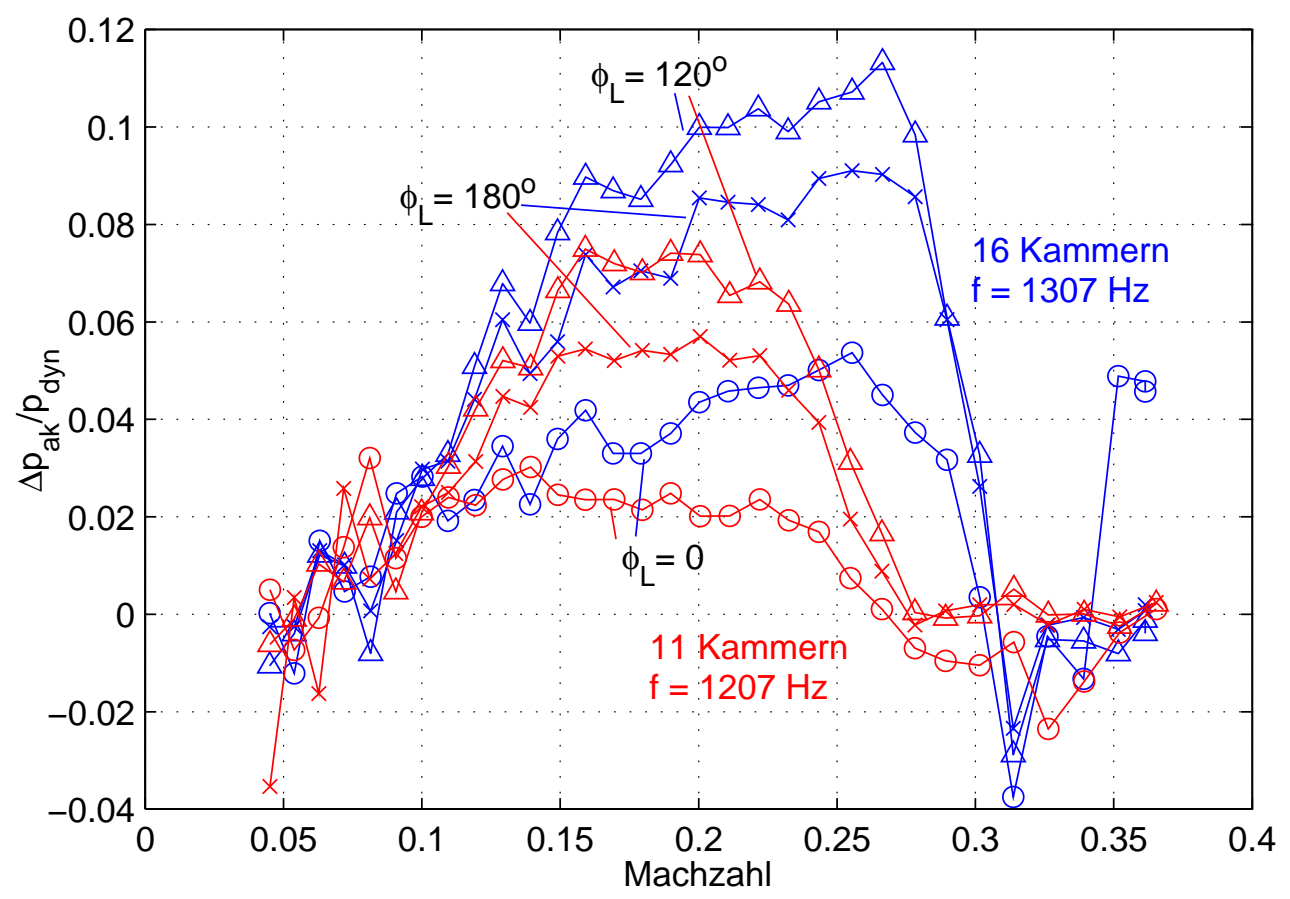

Abb. 2.31: Druckabfall $\Delta p_{\mathrm{ak}} / p_{\text {dyn }}$ am Resoantorabschnitt mit elf Resonatorkammern bei $1207 \mathrm{~Hz}$ bzw. 16 Kammern bei $1307 \mathrm{~Hz}$ in Abhängigkeit von der Machzahl, jeweils mit gleichphasiger $\left(\times, \phi_{L}=180^{\circ}\right)$, zirkularer $\left(\triangle, \phi_{L}=120^{\circ}\right)$ und gleichphasiger Beschallung $\left(\circ, \phi_{L}=0^{\circ}\right)$.

Zum Vergleich des Druckabfalls mit dem akustischen Verhalten ist bei den 16 Kammern der Schalldruck zur Messung $m=1$ gezeigt, der in der letzten Resonatorkammer gemessen wurde. Bei der Messung mit nur elf Kammern war diese abgedeckt, die entsprechende Messkurve ist hier also nicht vorhanden. Demnach liegen die Maxima der Schalldrücke tendenziell bei etwas tieferen Frequenzen als das Maximum im Druckabfall. Um das Nebenmaximum bei $550 \mathrm{~Hz}$ ist der Schalldruck in der Resonatorkammer kaum erhöht.

Die beiden höheren Maxima im Druckabfall sind unabhängig von der Machzahl. Daher kann bei ihren Frequenzen einfach die Abhängigkeit des Druckabfalls von der Machzahl gemessen werden. Abbildung 2.31 zeigt den Druckabfall für 16 Resonatorkammern bei $1307 \mathrm{~Hz}$ und für elf Resonatorkammern bei $1207 \mathrm{~Hz}$ in Abhängigkeit von der Machzahl für die drei Beschallungsarten. Mit elf Kammern steigt $\Delta p_{\mathrm{ak}} / p_{\text {dyn }}$ bis ca. Ma $=0,15$ an und bleibt dann bis $\mathrm{Ma}=0,24$ etwa konstant. Für größere Strömungsgeschwindigkeiten fällt der Wert wieder ab. Bei der Anregung mit zwei Lautsprechern ist der Druckabfall um etwa $20 \%$ verringert im Vergleich zu der Anregung mit drei Lautspre- 


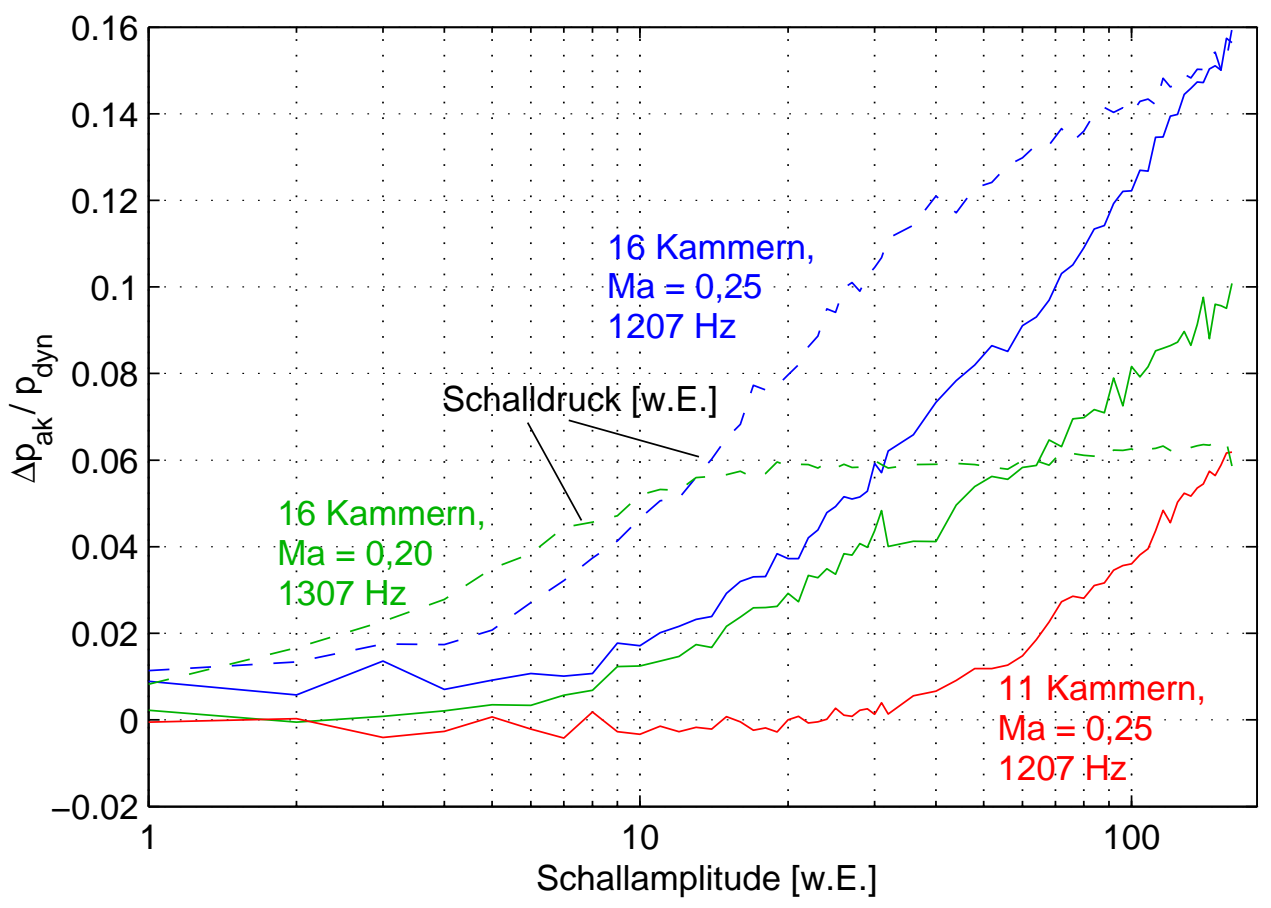

Abb. 2.32: Druckabfall $\Delta p_{\text {ak }} / p_{\text {dyn }}$ für den Resonatorabschnitt mit 16 bzw. elf Resonatoren in Abhängigkeit von der Lautsprecheramplitude für nicht abstrahlende Beschallung $\phi_{L}=120^{\circ}$. Messungen für 16 Resonatoren mit $\mathrm{Ma}=0,2$ und $1307 \mathrm{~Hz}$ und mit $\mathrm{Ma}=0,25$ und $1207 \mathrm{~Hz}$, sowie für elf Resonatoren mit $\mathrm{Ma}=0,25$ und $1207 \mathrm{~Hz}$. Zum Vergleich sind die Amplituden des Schalldrucks in der letzten der 16 Kammern gestrichelt dargestellt.

chern.

Bei den 16 Resonatoren reicht der Anstieg bis zu Ma $=0,27$, fällt dann steil ab und erreicht für $\mathrm{Ma}=0,31$ deutlich negative Werte. Bei dieser Machzahl-FrequenzKombination wird die turbulent angeregte Instabilität gut unterdrückt und der sonst durch die globale Instabilität vorhandene Druckabfall vermindert.

Der Druckabfall hängt wesentlich von der Amplitude des anregenden Schalls ab. Wie in Abb. 2.23 schon für die Anregung mit einer ebenen Welle $(m=0)$ ist in Abb. 2.32 die Abhängigkeit für die Mode $m=1\left(\phi_{L}=120^{\circ}\right)$ gezeigt. Gemessen wurde für 16 Kammern bei Ma $=0,25$ mit $1207 \mathrm{~Hz}$ und bei $\mathrm{Ma}=0,2$ mit $1307 \mathrm{~Hz}$, sowie für elf Kammern bei $\mathrm{Ma}=0,25$ mit $1207 \mathrm{~Hz}$.

Das Verhalten ist hier ähnlich dem Verhalten bei einer ebenen Welle. Wenn eine bestimmte Amplitude überschritten ist, steigt bei exponentiellem Anwachsen der An- 


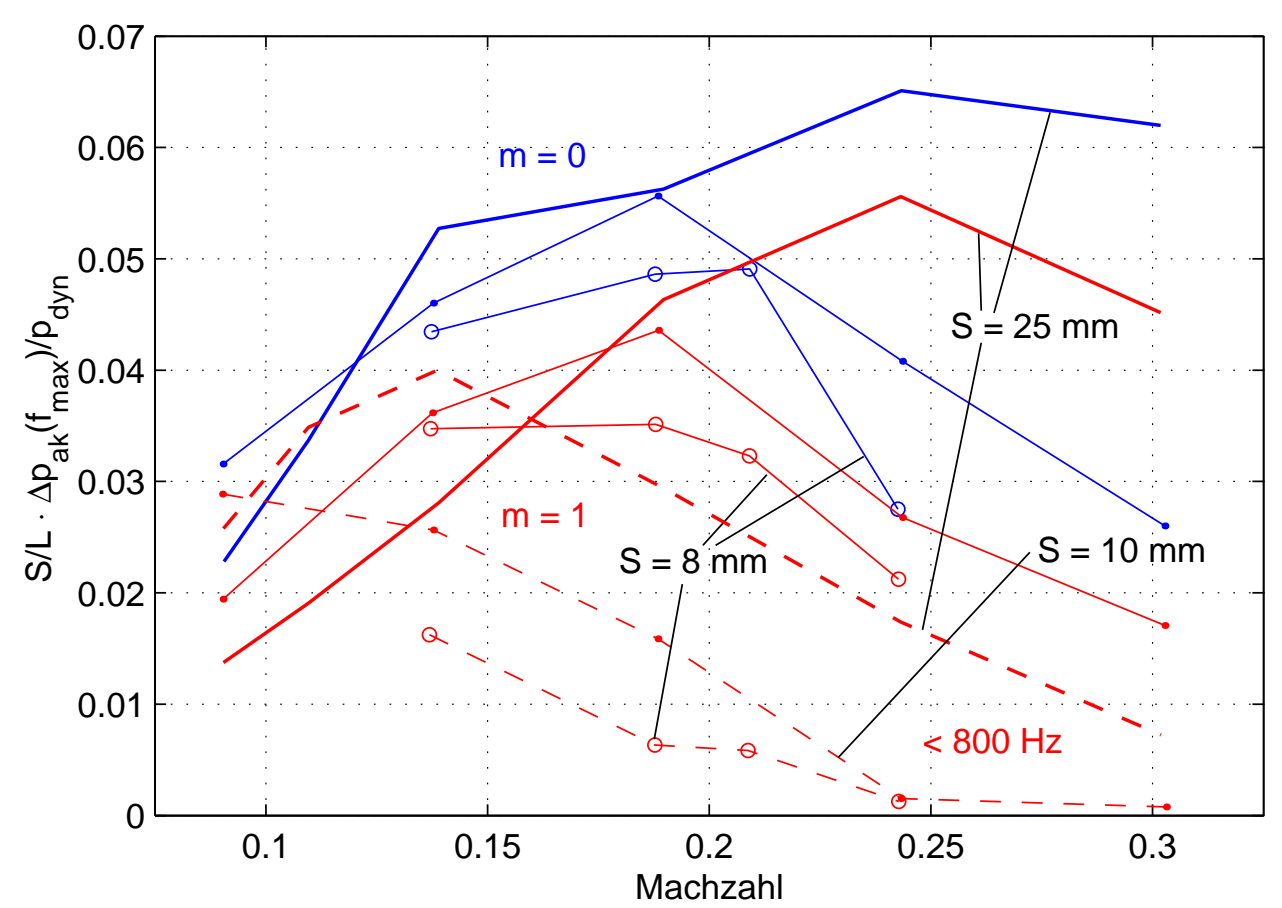

Abb. 2.33: Maximal erreichter schallinduzierter Druckabfall $\frac{S}{L} \Delta p_{\text {ak }} / p_{\text {dyn }}$ am Abschnitt mit 16 Resonatoren ohne $(S=25 \mathrm{~mm})$ und mit Zentralkörper $(S=$ $10 \mathrm{~mm}$ und $S=8 \mathrm{~mm}$ ) bei Anregung der Moden $m=0$ (blau, $\phi_{L}=0^{\circ}$ ) und $m=1\left(\operatorname{rot}, \phi_{L}=120^{\circ}\right)$. Für die Anregung $\phi_{L}=120^{\circ}$ ist das lokale Maximum unterhalb von $800 \mathrm{~Hz}$ gestrichelt eingezeichnet.

regung der Druckabfall linear an. Maximal werden hier $0,16 p_{\text {dyn }}$ bei $\mathrm{Ma}=0,25$ mit 16 Kammern erreicht, was einer Verdopplung des Druckabfalls ohne Schall entspricht, vgl. Abb. 2.21.

Der Schalldruck wächst zunächst ähnlich wie der Druckabfall, nähert sich dann aber einer Konstanten an. Der Druckabfall steigt dagegen weiter mit der Anregungsamplitude.

Eine Zusammenfassung über den maximal erreichten Druckabfall von frequenzabhängigen Messungen gibt Abb. 2.33. Die ausgewerteten Messreihen wurden größtenteils bereits in Abb. 2.24 und Abb. 2.29 gezeigt. Für die ebene Mode $m=0$ (blau) und die Mode $m=1$ (rot) ist für verschiedene Zentralkörper in Abhängigkeit von der Machzahl der schallinduzierte Druckabfall von der jeweiligen Frequenz aufgetragen, bei der er maximal ist. Da die Mode $m=1$ zwei unterschiedliche Maxima hat, ist hier der Frequenzbereich aufgeteilt in einen Bereich tiefer Frequenzen unterhalb $800 \mathrm{~Hz}$ (gestrichelt) und einen Bereich darüber bis 1,6 kHz. Um die Streuung der Werte et- 
was abzumildern, ist jeder hier gezeigte Messpunkt aus einer Mittelung über die drei größten Werte gewonnen.

Mit der Mode $m=0$ kann der größte Druckabfall erzeugt werden. Bei freiem Querschnitt $(S=25 \mathrm{~mm})$ nimmt $\Delta p_{\mathrm{ak}}$ bis $\mathrm{Ma}=0,24 \mathrm{zu}$. Zwischen $\mathrm{Ma}=0,14$ und $\mathrm{Ma}=0,24$ ist der akustisch verursachte, zusätzliche Druckabfall etwa doppelt so groß wie der Druckabfall in dem Resonatorabschnitt ohne eingebrachten Schall. Bei größerer Machzahl wird der Druckabfall wieder kleiner. Für kleinere $S$ wird das Maximum schon bei kleineren Machzahlen erreicht. Das Verhalten für die nicht abstrahlende Anregung verläuft bei den höheren Frequenzen ganz ähnlich (durchgezogene rote Linien), nur sind die Kurven etwas nach unten verschoben.

Der Druckabfall bei $m=1$ und den tieferen Frequenzen unterhalb $800 \mathrm{~Hz}$ hat sein Maximum mit $\mathrm{Ma}=0,14$ bei kleineren Machzahlen. Für Ma = 0,09 kann der Druckabfall am Resonatorabschnitt mit dieser Mode noch mehr als verdoppelt werden. 


\section{Kapitel 3}

\section{Eigenschaften des rechteckigen Resonatorabschnitts}

\subsection{Aufbau des Resonatorabschnitts}

Im vorigen Kapitel wurden die akustischen Eigenschaften überströmter radialsymmetrischer Resonatorabschnitte untersucht. Neben runden Rohren kommen in technischen Anwedungen häufig Kanäle mit rechteckigem Querschnitt vor. Dieses Kapitel beschäftigt sich mit solch einem Kanalstück, das in Abb. 3.1 schematisch dargestellt ist. Es ist mittels zweier stetiger Übergangsstücke an dem schon zuvor verwendeten, runden Rohr befestigt. Der freie Querschnitt beträgt $30 \mathrm{~mm} \times 40 \mathrm{~mm}$. Die beiden längeren, gegenüberliegenden Seite bestehen aus jeweils elf Resonatorkammern, die $T=107 \mathrm{~mm}$ tiefe $\lambda / 4$-Resonatoren bilden. In Strömungsrichtung sind sie $B=4 \mathrm{~mm}$ ausgedehnt und durch $1 \mathrm{~mm}$ breite Bleche voneinander getrennt. Eine solche Resonatorkammer hat die mittlere Wandadmittanz

$$
Y_{\mathrm{W}}^{\prime}=-\frac{i}{\rho c} \tan \left(k_{y} T\right) .
$$

Die Dämpfung an der Wand der Resonatorkammern wird wie im runden Fall behandelt, es ist $k_{y}=k_{r, \mathrm{~W}}$ aus Gleichung 2.3. Die Resonatoren sind mit dem gleichen Drahtgitter wie der runde Abschnitt bedeckt. Bezieht man die zusätzliche Impedanz des Gitters sowie die Breite der Trennbleche in die Mittelung ein, wird die mittlere Wandadmittanz des Resonatorabschnitts

$$
Y_{W}=\frac{Y_{W}^{\prime}}{1,25 \cdot\left(1+0,04 \rho c Y_{W}^{\prime}\right)} .
$$

Die ersten drei Resonanzen der Wandauskleidung liegen bei $780 \mathrm{~Hz}, 2360 \mathrm{~Hz}$ und $3950 \mathrm{~Hz}$. Die erste Quermode in einer Resonatorkammer (in Richtung der $40 \mathrm{~mm}$ Ausdehnung) kann sich ab $4230 \mathrm{~Hz}$ ausbilden. 
perspektivische Ansicht

Seitenansicht

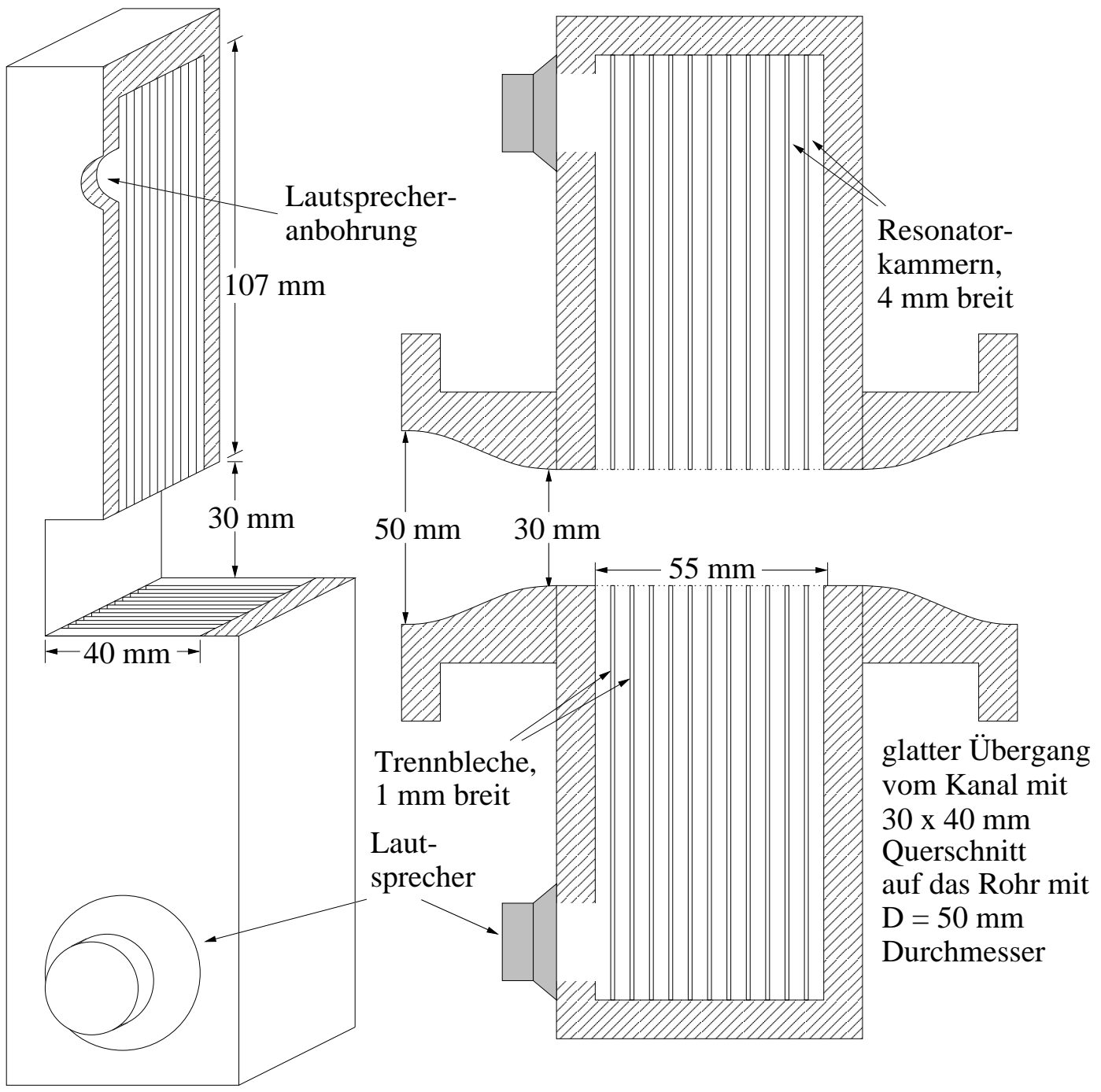

Abb. 3.1: Rechteckiger Resonatorabschnitt mit elf Resonatorkammern, die über stetige Übergänge an das runde Rohr angeschlossen sind. 
Die beiden sich gegenüberliegenden Wände sind mit diesen Resonatoren versehen. Für einige Messungen mit Resonatoren an nur einer Wand ist eine der beiden Seiten mit Klebestreifen abgedeckt.

Die angegebenen Machzahlen beziehen sich im Folgenden auf den rechteckigen Abschnitt, genauer auf dessen stromauf liegende Seite.

\subsection{Verhalten bei Strömung}

\subsubsection{Spektrales Verhalten}

Zunächst soll wieder das Spektrum stromab des Resonatorabschnitts betrachtet werden. Abb. $3.2 \mathrm{a}$ stellt den Pegelbereich zwischen $0 \mathrm{~dB}$ und $40 \mathrm{~dB}$ oberhalb des Median farbcodiert für Machzahlen bis 0,37 dar (vgl. Kreisquerschnitt, Abb. 2.6a). In zwei Frequenzbereichen treten Instabilitäten hervor. Mit A ist der Bereich der Instabilität etwas oberhalb der 1. Tiefenresonanz bezeichnet, mit B der Bereich etwas oberhalb der 2. Tiefenresonanz. Zwischen $\mathrm{Ma}=0,1$ und $\mathrm{Ma}=0,18$ ist $\mathrm{A}$ dominant, dann wird dieser Bereich zugunsten von B schwächer. Oberhalb von $\mathrm{Ma}=0,31$ wird er wieder stärker, die Amplituden bleiben aber kleiner als im Bereich B.

Wie beim runden Resonatorabschnitt ist die Instabilität an die Resonanzen der Wand gebunden, die Frequenz wächst auch hier mit der Machzahl an. Aber schon hier werden einige Unterschiede deutlich. So beginnt die Instabilität bei kleinen Machzahlen vergleichsweise knapp oberhalb der Resonanz. Dann ist nicht die Instabilität um die erste Resonanz im größten Machzahl-Bereich dominant, sondern um die zweite Resonanz, sie setzt bei $\mathrm{Ma}=0,17$ bei Frequenzen leicht unterhalb dieser Resonanz ein. Die Überhöhungen ändern sich nicht monoton mit der Machzahl, die Instabilität um A ist von $\mathrm{Ma}=0,2$ bis 0,3 relativ schwach.

Abb. 3.2b zeigt farbcodiert die Phase der Kohärenz $\gamma_{W_{1} W_{2}}$ zwischen den Mikrofonwerten, die in den gegenüberliegenden, stromabseitigen Resonatorkammern aufgenommen wurden, vgl. Abschnitt 2.6. Wenn der Betrag der Kohärenz kleiner als 1/3 ist, sind die Werte blass gezeichnet.

Im Frequenzbereich A mit $\mathrm{Ma}<0,19$, der relativ viel Energie abstrahlt, ist hier keine Kohärenz zu erkennen; für größere Machzahlen sind die Signale gleichphasig. Der Übergang in diesem Spektralbereich zu gleichphasigen Signalen tritt erstaunlicherweise gerade dort ein, wo diese Frequenzkomponente schwächer abgestrahlt wird. 

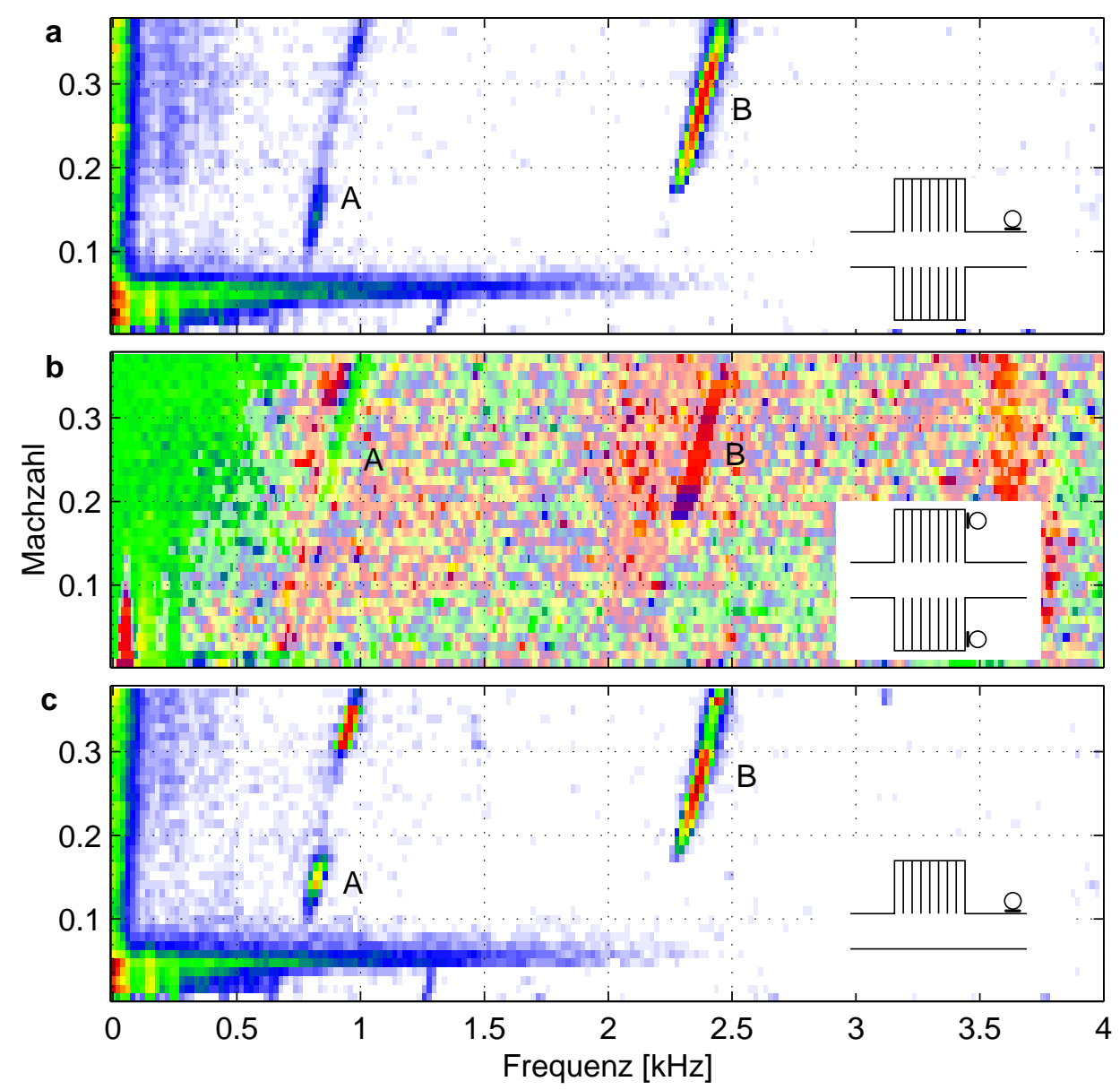

Abb. 3.2: Farbcodierte Spektren für Machzahlen bis 0,37 stromab elf rechteckiger Resonatoren auf beiden Kanalseiten (a), sowie die Phase der Signale zwischen den letzten Resonatoren (b) und das Spektrum stromab einer auf einer Seite mit Resonatoren versehenen Wand (c). 
Der Bereich $\mathrm{B}$ weist für $\mathrm{Ma} \approx 0,2$ eine Phasenverschiebung von etwa $90^{\circ}$ zwischen den gegenüberliegenden Resonatorkammern auf. Durch die turbulenten, statistischen Druckschwankungen können die Signale im Mittel nur gleich- oder gegenphasig sein, wenn keine Symmetriebrechung vorliegt. Bei größeren Strömungsgeschwindigkeiten werden die Signale gegenphasig. Auch das war nicht zu erwarten, da in diesem Frequenzbereich viel Energie abgestrahlt wird. Da gegenphasige Signale nicht ins Rohr abstrahlen würden, muss noch eine kleinere gleichphasige Komponente vorliegen.

Insgesamt fällt auf, dass im überwiegenden Frequenzbereich für alle Machzahlen die Signale im Vergleich zum runden Resonatorabschnitt nur sehr geringe Kohärenz aufweisen, siehe Abb. 2.26. Bei tiefen Frequenzen sind die Signale kohärenter und gleichphasig. Die Druckschwankungen in den gegenüberliegenden Resonatorkammern des Rechteckkanals sind im Vergleich zum runden Resonatorabschnitt weitgehend unabhängig voneinander. Dort waren die Kammern nicht in Winkelrichtung unterteilt, und die Mikrofone in der Kammer damit akustisch durch vorwiegend ruhende Luft miteinander verbunden. Hier dagegen müssen kohärente Signale den durchströmten Bereich durchlaufen.

In Abb. $3.2 \mathrm{c}$ ist das Spektrum eines an nur einer Wand mit Resonatoren versehenen Abschnitts dargestellt; die gegenüberliegenden Resonatoren sind mit einem Klebestreifen abgedeckt. Die Frequenzbereiche der Instabilitäten mit einer und mit zwei Wänden, die mit Resonatorkammern versehen sind, verhalten sich weitgehend gleich. Allerdings ist im ersten Fall insbesondere die Instabilität um die erste Resonanz etwas stärker ausgeprägt als um die zweite Resonanz, vgl. Abb. 3.2a, c. Die Amplitude in diesem Frequenzbereich ist von $\mathrm{Ma}=0,18$ bis $\mathrm{Ma}=0,3$ klein.

Die Ähnlichkeit im Spektrum zu Abb. 3.2a deckt sich mit der Feststellung, dass die Druckschwankungen der gegenüberliegenden Kammern weitgehend unabhängig sind.

\subsubsection{Amplitudenstatistik der Druckschwankungen}

Abb. 3.3 zeigt einen Vergleich der Verteilung der Mikrofonmesswerte mit einer Normalverteilung stromab des Resonatorabschnitts mit beidseitig gekammerten Wänden. Die Auswertungsmethode ist in Abschnitt 2.3.4 beschrieben.

Die Instabilität ist hier für kleine Strömungsgeschwindigkeiten im Frequenzbereich meist schmaler als die des runden Resonatorabschnitts. Ein so schmalbandiges Pfeifen wie im Fall der runden Kammern um $1,2 \mathrm{kHz}$, kommt jedoch nicht zustande. Die maximale Abweichung von einer Normalverteilung liegt hier bei den Machzahlen, in denen die 2. Resonanz dominant ist. Die Verteilung bleibt einer Normalverteilung deutlich ähnlicher als die des runden Abschnitts mit größeren Machzahlen. 


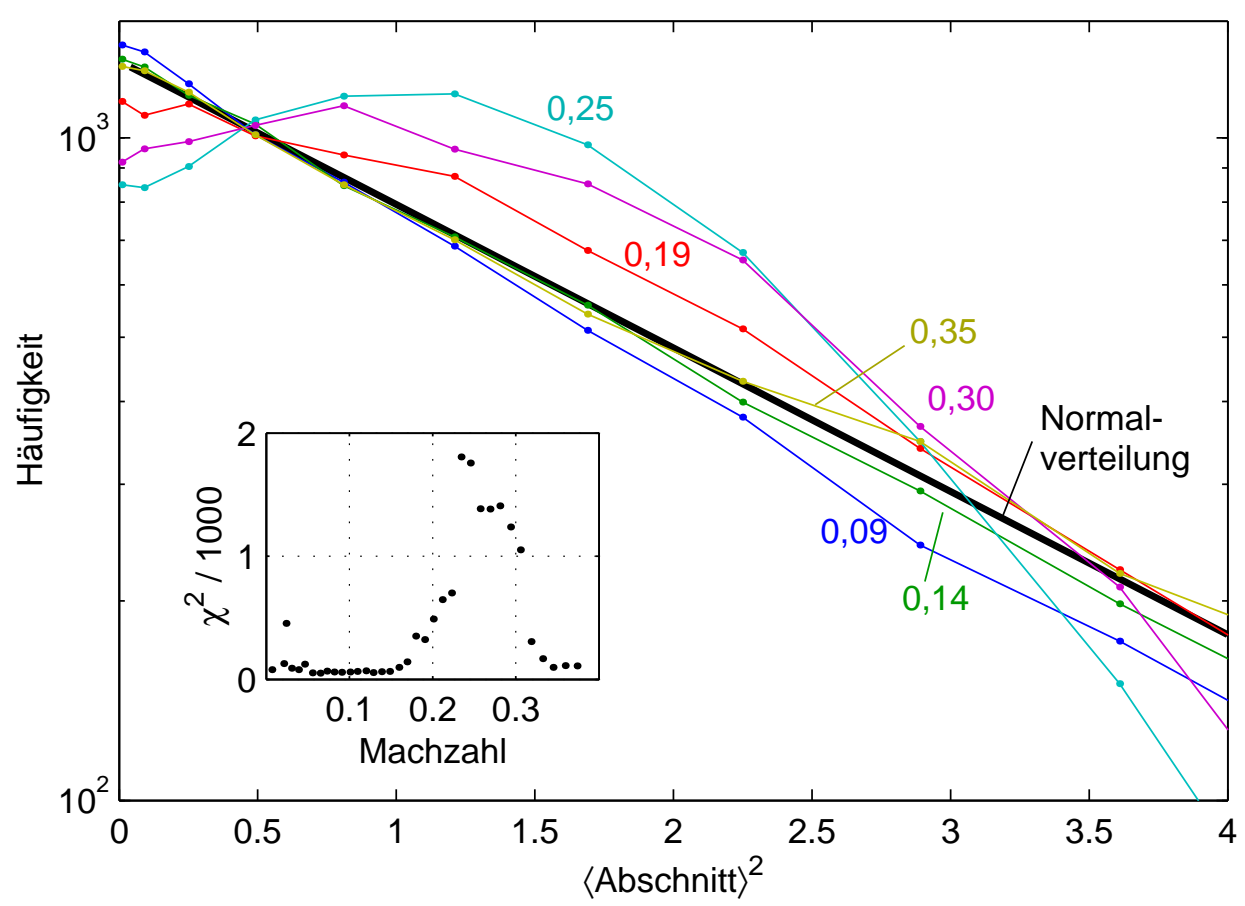

Abb. 3.3: Verteilung des Betrags der auf den RMS-Wert normierten Samples der Mikrofonsamples $\left|w_{i}\right| / \sigma$, vgl. Abb. 2.9, Abschnitt 2.3.4.

\subsection{Einfluss von Schall auf den Druckabfall}

Abb. 3.4 zeigt den schallinduzierten Druckabfall $\Delta p_{\text {ak }} / p_{\text {dyn }}$ sowie Mikrofonmesswerte im Rohr stromauf, im Rohr stromab und in den hinteren Resonatorkammern als Funktion der Amplitude des anregenden Schalls. Angeregt wird die Instabilität für $\mathrm{Ma}=0,15$ bei $822 \mathrm{~Hz}$. Die fett gezeichnete Kurve gibt den Druckabfall in der Strömung an, der durch den Schall verursacht wird, wenn zwei Lautsprecher ${ }^{1}$ an der ersten Resonatorkammer gleichphasig (blau) bzw. gegenphasig (rot) betrieben werden. Die übrigen Kurven geben die Mikrofonamplituden im Frequenzbereich um $822 \mathrm{~Hz}$ an verschiedenen Positionen mit der gleichen Farbcodierung wieder. Die durchgezogenen Kurven sind mit einem Mikrofon stromab gemessen, die gestrichelten je in einer stromabseitigen Resonatorkammer, und die strichpunktierte ist stromauf gemessen.

Bei gegenphasiger Anregung sind die Werte stromab und stromauf sehr klein, da die Anregung so adaptiert wurde, dass möglichst wenig Signal in die Messrohre eindringt. Die Messwerte dieser Mikrofonpositionen zeigen nur den Erfolg der Kompensation. Interessanter sind die Kurven zur gleichphasigen Beschallung.

${ }^{1}$ IMG StageLine, 40 Watt, $8 \Omega$ 


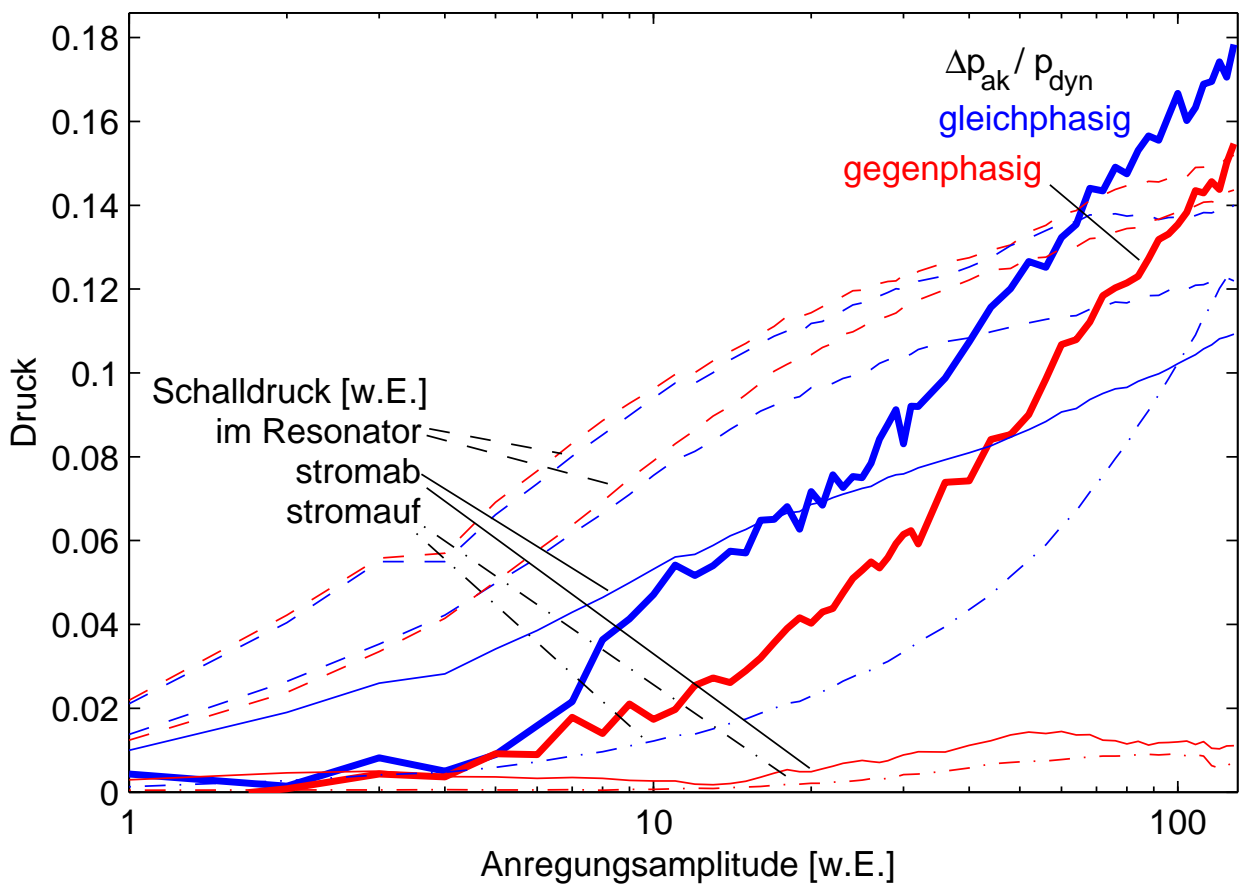

Abb. 3.4: Druckabfall $\Delta p_{\mathrm{ak}} / p_{\mathrm{dyn}}$ in Abhängigkeit von der Lautsprecheramplitude für gleichphasige (blau) und gegenphasige Beschallung (rot) bei Ma = 0,15 und $822 \mathrm{~Hz}$, sowie der Schalldruck an verschiedenen Positionen.

Der Schalldruck stromauf wächst (bei gleichphasiger Anregung) linear mit der Lautsprecheramplitude, also in der halblogarithmischen Darstellung exponentiell. Stromab dagegen steigt die Schallamplitude linear bei exponentiell wachsender Anregung. Damit ist die Instabilitätswelle schon in der Sättigung. In den hinteren Resonatorkammern wächst der Schalldruck zunächst stärker an als im Rohr stromab, geht aber bei größeren Amplituden in eine Sättigung. Die Kurven für gleich- und für gegenphasige Beschallung verlaufen hier annähernd gleich. Der Schalldruck in den stromabseitigen Resonatorkammern ist ähnlich dem Verlauf im runden Resonatorabschnitt, vgl. Abb. 2.32.

Auch der statische Druckabfall ist vergleichbar. Für sehr kleine Amplituden ist zunächst kein Effekt zu sehen. Ab einer Grenzamplitude steigt dann der Druckabfall mit dem Logarithmus der Anregung linear an, sowohl für gleich- als auch für gegenphasige Beschallung. Auffallend dabei ist, dass der Druckeinfluss gleichphasig stärker ist, während in den Resonatoren die Schallamplitude gegenphasig etwas größer wird.

Die Abhängigkeit des Druckabfalls von der Frequenz um die beiden ersten Resonanzen ist in Abb. 3.5 aufgetragen. Die Machzahl reicht von 0,15 bis 0,3, die Lautsprecher an 


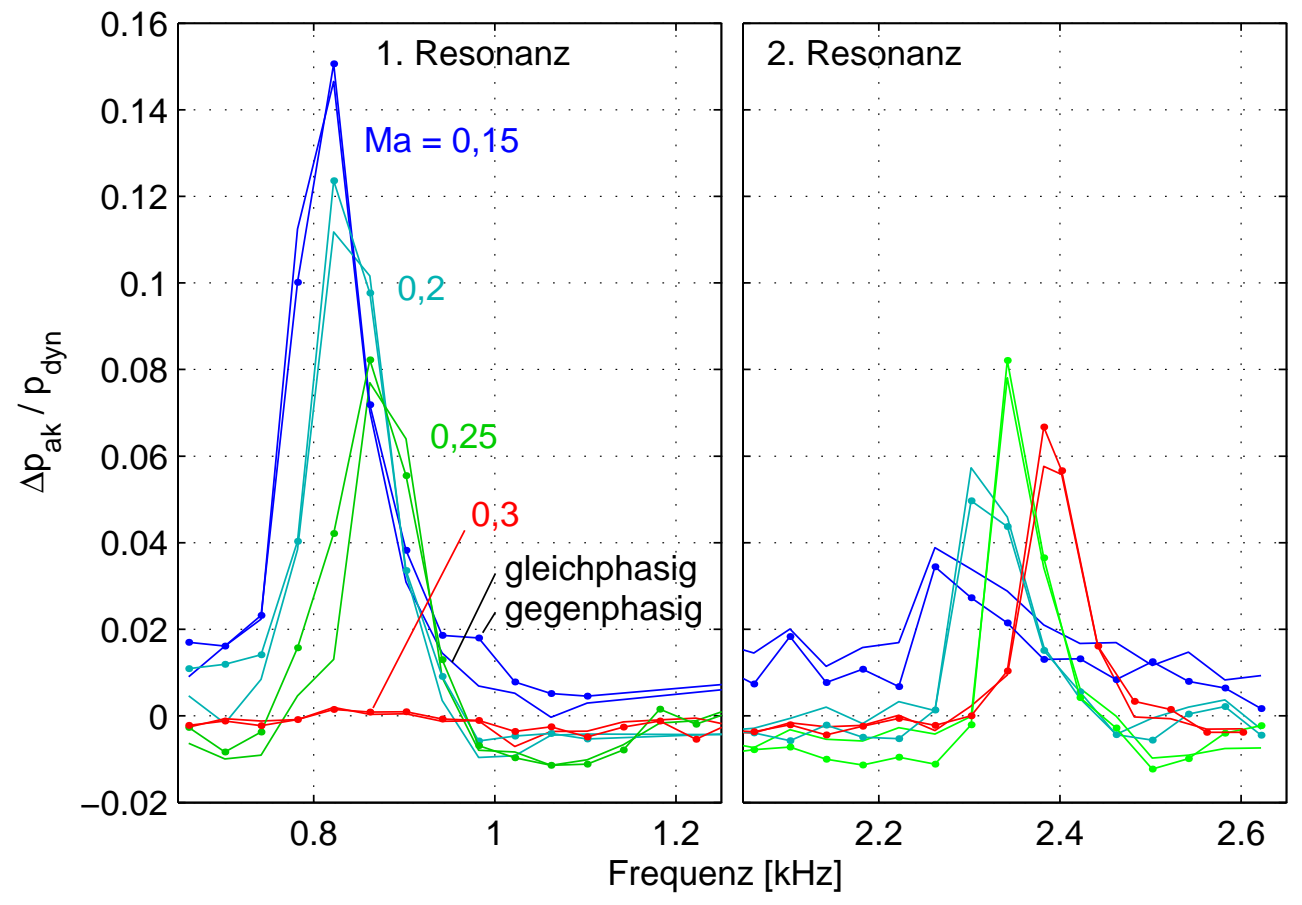

Abb. 3.5: Schallinduzierter Druckabfall für gleich- und gegenphasige Anregung um die ersten beiden Resonanzen bei verschiedenen Strömungsgeschwindigkeiten.

den Resonatorkammern werden gleichphasig und gegenphasig (ohne bzw. mit Punkten an den zugehörigen Messwerten) betrieben.

Die Kurven für die beiden Beschallungsarten zeigen keine signifikanten Unterschiede. Mit steigender Machzahl nimmt um die erste Resonanz der Druckabfall $\Delta p_{\text {ak }} / p_{\text {dyn }}$ ab, bei $\mathrm{Ma}=0,3$ ist kein Einfluss mehr zu erkennen. Die Frequenz, bei der der maximale Druckabfall erreicht wird, nimmt mit der Machzahl (entsprechend der Instabilität im Spektrum) zu. Im Spektrum gab es allerdings keinen Hinweis, dass bei $\mathrm{Ma}=0,3$ kein Druckabfall mehr zu erzielen ist.

Um die zweite Resonanz stimmt die Größe des erreichbaren Druckabfalls besser mit der Überhöhung im Spektrum überein. Bei Ma =0,15 ist im Spektrum noch kein Effekt zu sehen, der Druckabfall ist hier auch am kleinsten. Der größte Druckabfall kommt bei $\mathrm{Ma}=0,25$ zustande, was nahe dem Maximum im Spektrum für die verschiedenen Machzahlen liegt.

Ein Vergleichswert für den Druckabfall ohne Schallanregung ist hier nicht vorhanden, da nicht zwischen dem Druckabfall durch die Eigenschaften der Wand und dem durch die Querschnittsänderung verursachten Druckabfall unterschieden werden kann. 


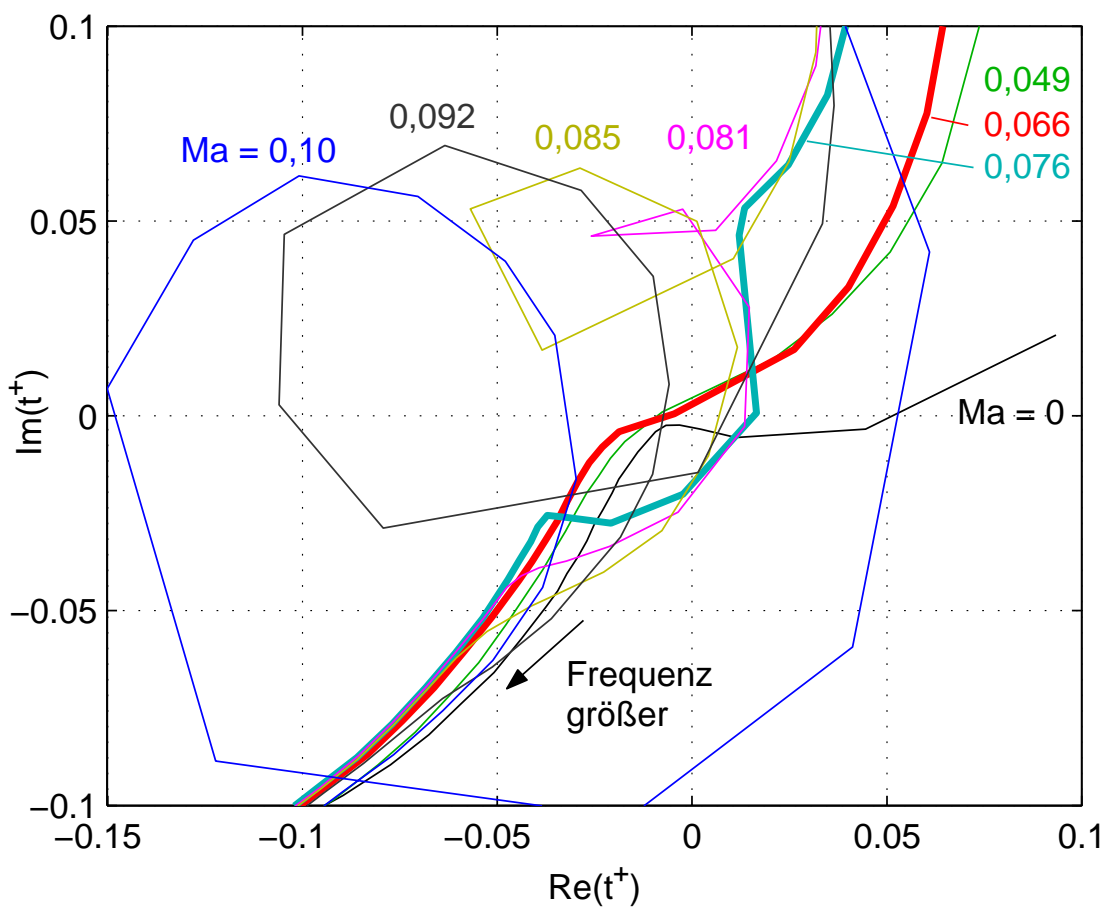

Abb. 3.6: Transmissionsfaktor $t^{+}$für elf Resonatorkammern in einer Kanalseite in der komplexen Ebene, mit der Machzahl als Parameter. Die Frequenz ist in $10 \mathrm{~Hz}-\mathrm{Sch}$ ritten zwischen 722 und $902 \mathrm{~Hz}$ variiert.

\subsection{Transmissionsfaktoren}

Im Folgenden werden die Transmissionsfaktoren $t^{+}$zu dem rechteckigen Kanalabschnitt mit elf Resonatoren sowohl an nur einer Kanalseite, als auch an beiden gegenüberliegenden Seiten gezeigt. Dabei bezieht sich $t^{+}$auf den Resonatorabschnitt inklusive der Übergänge vom runden zum rechteckigen Rohr.

Zunächst ist in Abb. 3.6 der Transmissionsfaktor $t^{+}$für den Resonatorabschnitt mit Resonatoren an nur einer Kanalseite in der komplexen Ebene aufgetragen. Die Frequenzen liegen um die erste Resonanz zwischen 722 und $902 \mathrm{~Hz}$. Die Machzahl wurde (analog dem runden Fall, Abb. 2.13) in kleinen Schritten erhöht. Zwischen den beiden fett gezeichneten Linien zu Ma $=0,066$ und 0,076 setzt die Instabilität ein. Ab $\mathrm{Ma}=0,081$ wird eine Schlaufe durchlaufen, die bis $\mathrm{Ma}=0,1$ zunehmend größer wird.

Der Betrag von $t^{+}$ist für größere Strömungsgeschwindigkeiten mit Resonatoren an einer Wand in Abb. 3.7 mit gestrichelten Linien aufgetragen und mit Resonatoren an beiden Wänden mit einer durchgezogenen Linie. Wegen des nichtlinearen Verhaltens für nicht ganz kleine Schallamplituden sind die Werte in der Instabilität wieder nur als 
untere Abschätzung von $t^{+}$zu sehen. Die Frequenzen liegen um die erste Resonanz. Für tiefe Frequenzen bei $650 \mathrm{~Hz}$ liegen die Werte beider Konfigurationen relativ dicht zusammen um $\left|t^{+}\right| \approx 0,5$. Oberhalb der Resonanz, um $1050 \mathrm{~Hz}$ sind sie aufgespalten: mit Resonatoren an einer Seite ist $\left|t^{+}\right| \approx 0,4$, sind sie an beiden Seiten ist $\left|t^{+}\right|$zwischen 0,1 und 0,2 .

Wie erwartet verläuft der einseitige Fall: Mit wachsender Machzahl nimmt die Verstärkung zu und verschiebt sich zu höheren Frequenzen. Ab Ma = 0,25 wird hier $\left|t^{+}\right|>1$, es tritt also Verstärkung auf. Bemerkenswert ist, dass die Überhöhung im Spektrum (Abb. 3.2c) nicht mit der Verstärkung korrespondiert: Für Ma = 0,2 und 0,25 kann die Instabilität von der 2. auf die 1. Resonanz gezogen werden.

Ungewöhnlich verlaufen dagegen die Kurven zu $\left|t^{+}\right|$mit Resonatoren an beiden Seiten. Bei Ma $=0,15$ ist die Überhöhung kleiner als bei einseitiger Resonatorwand, bei $\mathrm{Ma}=0,2$ ist kaum noch ein Maximum zu erkennen, bei $\mathrm{Ma}=0,25$ ist es gänzlich verschwunden. Die spektralen Überhöhungen, die in Abb. 3.2 a gezeigt wurden, deuten zwar auf ein solches Verhalten hin, jedoch ist bei dem Kanalabschnitt mit Resonatoren an nur einer Seite auch eine Verstärkung vorhanden, wenn keine Überhöhung im Spektrum zu sehen ist.

Über die Ursachen kann nur spekuliert werden. Wenn die Instabilitäten lokal an jeder Wand und mit schneller Phasenänderung laufen, könnten sich so bis zur letzten Resonatorkammer gegenphasige Wellen eingestellt haben, die nicht nennenswert abstrahlen. Auf eine Phasenabweichung deutet auch Abb. $3.2 \mathrm{~b}$ hin - allerdings für alle Messungen mit Resonatorkammern an zwei Kanalwänden.

In Abb. 3.8 ist $\left|t^{+}\right|$um die 2. Resonanz aufgetragen, wie zuvor sowohl mit Resonatorkammern an einer als auch an beiden Wänden für verschiedenen Strömungsgeschwindigkeiten. In beiden Fällen wird hier ab $\mathrm{Ma}=0,2$ ein Transmissionsfaktor von eins überschritten. In der Instabilität verlaufen die Kurven für größere Machzahlen sehr ähnlich. Für Frequenzen oberhalb der Instabilität um $2,55 \mathrm{kHz}$ ist der Transmissionsfaktor zu den Resonatoren an einer Seite mit $\left|t^{+}\right| \approx 0,5$ etwa fünfmal so groß wie bei beidseitigen Resonatoren. 


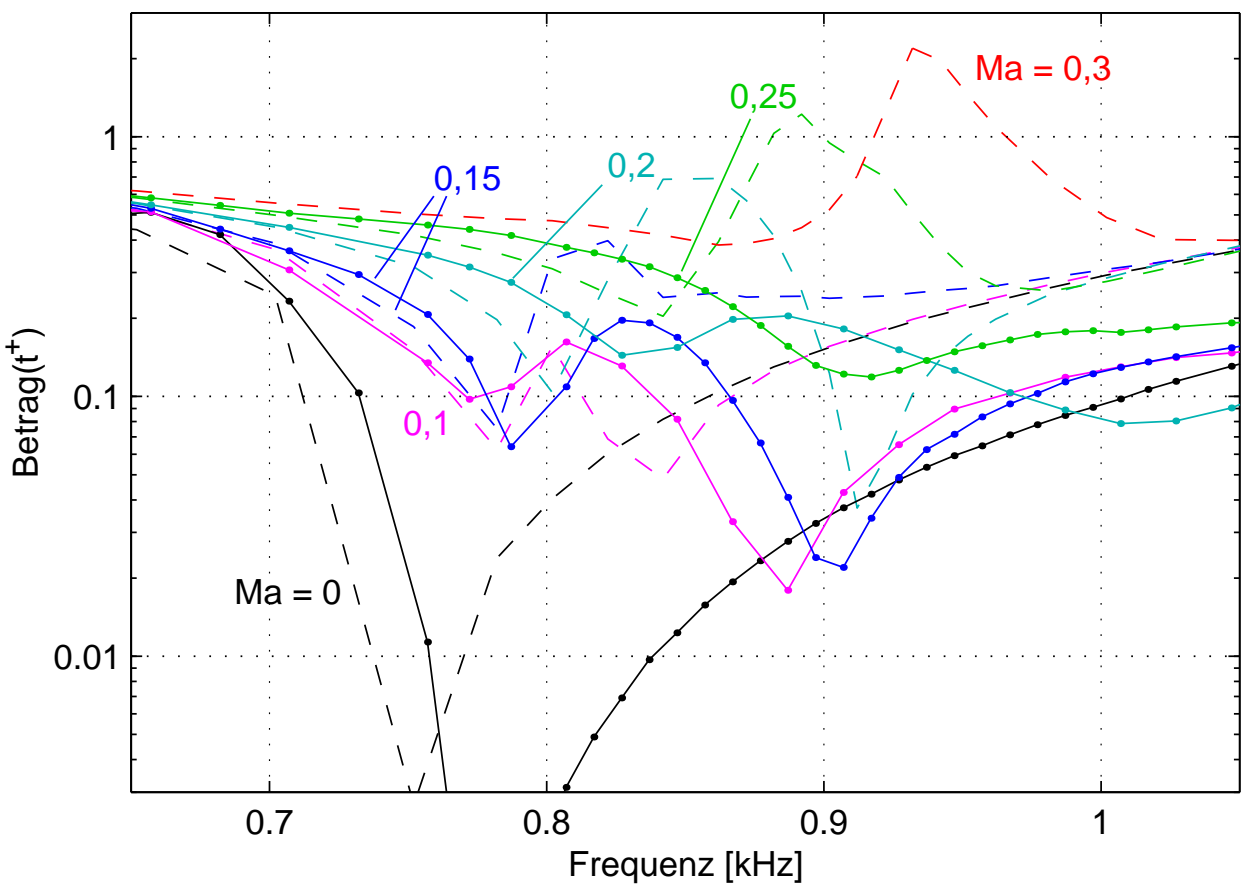

Abb. 3.7: Betrag des Transmissionsfaktors $t^{+}$um die erste Resonanz, mit der Machzahl als Parameter, für elf Resonatorkammern an einer (gestrichelt) und beiden Kanalseiten (durchgezogene Linie).

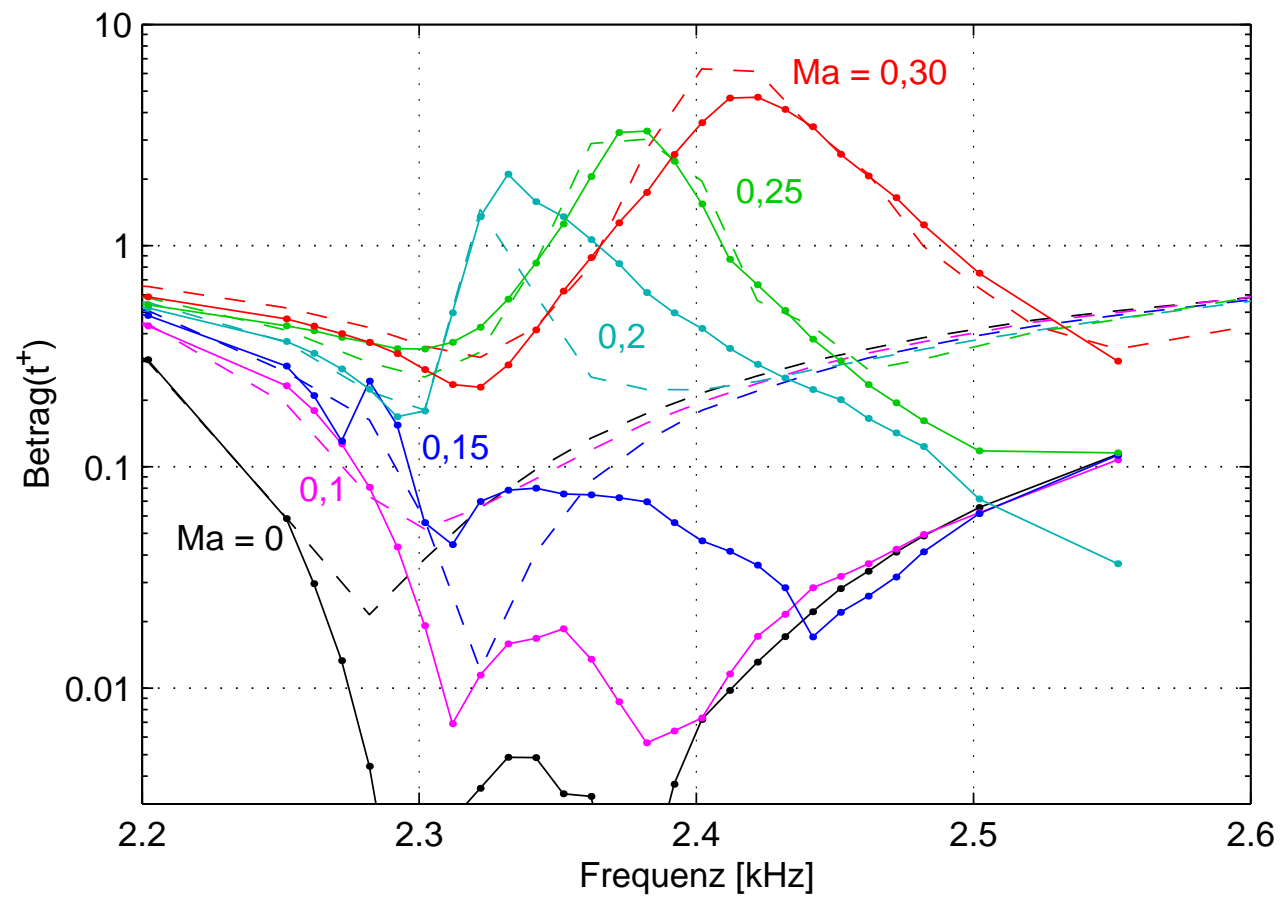

Abb. 3.8: Betrag des Transmissionsfaktor $t^{+}$, wie oben, nur um die zweite Resonanz. 


\section{Kapitel 4}

\section{Theorie zur Schallausbreitung im Resonatorabschnitt}

Hier sollen theoretische Überlegungen zur Schallausbreitung im runden Resonatorabschnitt ohne Zentralkörper diskutiert werden. Die Vorgehensweise ist ähnlich der von Großer [10, Kap. 3, 4] und Rienstra [21]. Es wird nicht die Sprungbedingungen zwischen dem hartwandigen Rohr und dem Resonatorabschnitt mit einer von Null verschiedenen Admittanz an der Rohrwand betrachtet, sondern nur die Dispersionsrelation untersucht, die das Verhalten im runden Resonatorabschnitt beschreibt.

Das Ziel bei diesem Ansatz ist es, eine Mode $k_{x}(\omega)$ zu finden, die in dem Frequenzbereich verstärkt wird, in dem auch im Experiment Schallverstärkung bzw. der zugeordnete schallinduzierte Druckabfall auftritt.

\subsection{Aufstellen der Dispersionsrelation}

Der Ausgangspunkt sind die Kontinuitätsgleichung und die Eulergleichungen in Zylinderkoordinaten. Linearisiert lauten sie

$$
\begin{aligned}
\frac{1}{c^{2}}\left(\partial_{t}+\bar{u} \partial_{x}\right) \frac{p}{\bar{\rho}}+\left(\partial_{r}+\frac{1}{r}\right) u_{r}+\frac{1}{r} \partial_{\phi} u_{\phi} & +\partial_{x} u_{x}=0 \\
\left(\partial_{t}+\bar{u} \partial_{x}\right) u_{r} & =-\partial_{r} \frac{p}{\bar{\rho}} \\
\left(\partial_{t}+\bar{u} \partial_{x}\right) u_{\phi} & =-\frac{1}{r} \partial_{\phi} \frac{p}{\bar{\rho}} \\
\left(\partial_{t}+\bar{u} \partial_{x}\right) u_{x}+\left(\partial_{r} \bar{u}\right) u_{r} & =-\partial_{x} \frac{p}{\bar{\rho}}
\end{aligned}
$$

Dabei sind $u_{r}$ die Radial-, $u_{x}$ die Axial- und $u_{\phi}$ die azimutale Schnelle, $p$ ist der Wechseldruck, $\bar{\rho}$ der Gleichanteil der Dichte und $\bar{u}=\bar{u}(r)$ das Profil der Gleichströmung. 
Mit dem Ansatz, dass sich die Wechselgrößen $\sim \exp \left(-i \omega t+i k_{x} x+i m \phi\right)$ verhalten, kann die Divergenz der Eulergleichungen in die Kontinuitätsgleichung eingesetzt werden. Die Gleichung lässt sich dann zu

$$
\frac{\omega^{\prime 2}}{c^{2}} p+\Delta p+\frac{2 k_{x} \partial_{r} \bar{u}}{\omega^{\prime}} \partial_{r} p=0
$$

umformen, wobei die dopplerverschobene Frequenz $\omega^{\prime}=\omega-k_{x} \bar{u}$ eingeführt wurde. Der Laplace-Operator lautet in Zylinderkoordinaten $\Delta=\partial_{r}^{2}+\frac{1}{r} \partial_{r}+\frac{1}{r^{2}} \partial_{\phi}^{2}+\partial_{x}^{2}$, bzw. mit dem harmonischen Ansatz $\Delta=\partial_{r}^{2}+\frac{1}{r} \partial_{r}-\frac{m^{2}}{r^{2}}-k_{x}^{2}$.

\subsubsection{Rechteckprofil}

Nimmt man ein rechteckiges Strömungsprofil an,

$$
\begin{aligned}
& \bar{u}(r)=\bar{u}_{0} \quad \text { für } \mathrm{r}<\mathrm{R} \\
& \bar{u}(r)=0 \quad \text { für } \mathrm{r}=\mathrm{R}
\end{aligned}
$$

gilt für dessen Ableitung $\partial_{r} \bar{u}=0$ und Gleichung 4.3 vereinfacht sich zu

$$
\frac{\omega^{\prime 2}}{c^{2}} p+\Delta p=0 \quad \text { für } \mathrm{r}<\mathrm{R} .
$$

Die Lösung dieser Gleichung bilden die Besselfunktionen $J_{m}\left(k_{r} r\right)$ und $Y_{m}\left(k_{r} r\right)$ mit $k_{r}= \pm \sqrt{\frac{\omega^{\prime 2}}{c^{2}}-k_{x}^{2}}$. Hier soll nur ein offenes Rohr ohne Zentralkörper betrachtet werden. Daher gilt in der Rohrmitte die Randbedingung $\partial_{r} p(r=0)=0$, die Lösungen beschränken sich auf die Besselfunktion $p \sim J_{m}$. Damit lässt sich über die radiale Komponente der Eulergleichung die Radialschnelle $u_{r}=-\frac{i}{\bar{\rho} \omega^{\prime}} \partial_{r} p \sim-\frac{m}{r} J_{m}\left(k_{r} r\right)+k_{r} J_{m-1}\left(k_{r} r\right)$ berechnen.

In diesem Modell springt die Strömungsgeschwindigkeit unstetig von ruhender Luft in den Resonatorkammern und an der Rohrwand auf die Strömungsgeschwindigkeit im Rohr. Die Auslenkung $\eta:=\frac{i}{\omega^{\prime}} u_{r}$ und der Wechseldruck $p$ sind an der unendlich dünnen Grenzschicht stetig (siehe z. B. Großer [10, Kap. 3]). Damit springt die Radialschnelle $u_{r}$ und auch die Feldadmittanz ${ }^{1}$ an der Wand um den Faktor $\omega / \omega^{\prime}$. Die Feldadmittanz ist an der Rohrwand

$$
\left.Y\right|_{r=R}=u_{r} / p=-\frac{i \omega}{\bar{\rho} \omega^{\prime 2}}\left(k_{r} \frac{J_{m-1}\left(k_{r} R\right)}{J_{m}\left(k_{r} R\right)}-\frac{m}{R}\right) .
$$

\footnotetext{
${ }^{1}$ Mit Feldadmittanz wird die Admittanz bezeichnet, das man bei der Berechnung des Wechselfeldes von der Rohrmitte ausgehend erhält. Dagegen gibt die Wandadmittanz die mittlere Admittanz an der Rohrseite der Resonatorkammern an, die ausgehend von der Außenseite der Kammern $r=R_{a}$ berechnet wird.
} 


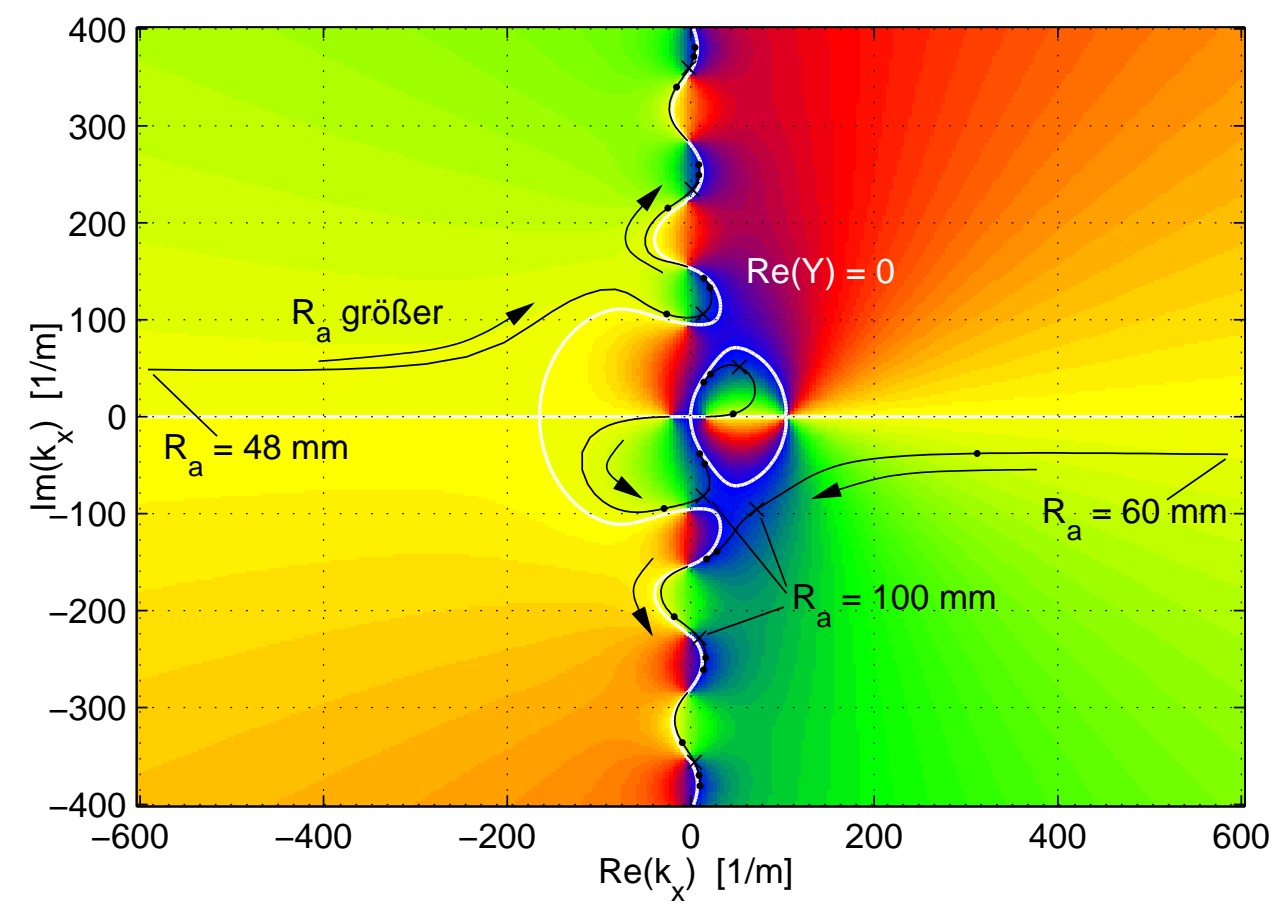

Abb. 4.1: Phase der Feldadmittanz $Y$ an der Rohrwand für ein Rechteckprofil bei $m=0$, Ma $=0,175$ und $f=1 \mathrm{kHz}$. Weiße Linie: $\operatorname{Re}(Y)=0$. Schwarz: Feldadmittanz vom Rohr aus ist gleich der Wandadmittanz nach Gleichung 2.4 mit $R_{a}$ von 25 bis $150 \mathrm{~mm}$. Punkte markieren $R_{a}=50,75,125$ und $150 \mathrm{~mm}$, Kreuze $R_{a}=100 \mathrm{~mm}$.

Im Druck und in der Schnelle geht zwar das Vorzeichen von $k_{r}$ ein, dass hier nicht festgelegt wurde. Bei der entscheidenden Größe, der Admittanz, lässt sich das Problem jedoch so formulieren, dass nur $k_{r}^{2}$ auftritt. Dies ist leicht zu sehen, wenn die Besselfunktion als Potenzreihe geschrieben wird.

Abb. 4.1 zeigt die Phase der Admittanz nach Gleichung 4.6 farbcodiert in der $k_{x^{-}}$ Ebene bei der Strömunggeschwindigkeit $\mathrm{Ma}=0,175$, der Frequenz $f=1 \mathrm{kHz}$ und der azimutalen Wellenzahl $m=0$ (Farbcodierung siehe Abb. 0.2 im Symbolverzeichnis).

Die Eigenschaften der Rohrwand sind soweit noch nicht berücksichtigt, hier geht die zweite Randbedinung ein. Lösungen der Dispersionsrelation (auch als Moden bezeichnet) sind die Punkte, in denen die Admittanz nach Gleichung 4.6 mit der Admittanz, die an der Wand vorliegt, übereinstimmt. Für ein hartes Rohr ist $Y=0$. Wenn an der Wand keine Dissipation vorhanden ist, so ist die Wandadmittanz rein imaginär, $\operatorname{Re}(Y)=0$. In der Auftragung verlaufen die weißen Kurven entlang dieser Wandadmittanz. 
Die Wandadmittanz für ein gekammertes Rohr ist in Gleichung 2.4 angegeben. Die schwarzen Kurven in Abb. 4.1 geben die Moden der Dispersionsrelation für eine gekammerte Wand mit Dämpfung an, bei der der Radius der Kammern vom Rohrradius $R_{a}=25 \mathrm{~mm}$ auf $R_{a}=150 \mathrm{~mm}$ anwächst. Die Moden sind für den Parameter $R_{a} \mathrm{im}$ Abstand von $25 \mathrm{~mm}$ mit Punkten gekennzeichnet. Kreuze markieren die Moden zu $R_{a}=100 \mathrm{~mm}$, dem Radius, der im Experiment vorhanden ist.

Bei der Änderung von $R_{a}$ geht die Wandadmittanz von schallhart über schallweich wieder fast auf den Ausgangspunkt der schallharten Wand zurück, wenn die Antiresonanz erreicht ist. An diesem Punkt müssen (bis auf kleine Dämpfungeffekte, die durch die Stokesdämpfung an den Kammerwänden und das Gitter oberhalb der Kammern zustande kommen) wieder die Moden existieren, mit denen begonnen wurde. In diesem Beispiel laufen die meisten Moden nicht für sich im Kreis, sondern sie wandern im Wesentlichen nach außen, zu betragsmäßig größeren $\operatorname{Im}\left(k_{x}\right)$. Die beiden entstehenden "Lücken" bei kleinen $\operatorname{Im}\left(k_{x}\right)$ werden durch zwei Moden aufgefüllt, die von betragsmäßig großen $\operatorname{Re}\left(k_{x}\right)$ kommen. Wird der Radius etwas weiter vergrößert, kommen die beiden Moden, die bei reellen $k_{x}$ begonnen haben, wieder fast auf ihrem Ausgangspunkt zurück.

Wenn der Außenradius $R_{a}$ deutlich größer als der Rohrradius ist, verlaufen die Kurven für eine kleine Radiusänderung ähnlich wie bei einer Frequenzänderung. Entscheidend ist, wie sich die Lage der betrachteten Frequenz zu der Resonanz verschiebt. In der Wandadmittanz ist der Faktor $k_{r, \mathrm{~W}} R_{a} \approx \frac{\omega}{c} R_{a}$ die entscheidende Größe.

In der Abbildung sind Wellenzahlen betragsmäßig bis maximal $\left|k_{x}\right|=600 \mathrm{~m}^{-1}$ dargestellt. Für größere Wellenzahlen ist die zugehörige Wellenlänge nicht mehr klein gegen die Breite der Resonatorkammern, die die Wand bilden. Die Wand reagiert dann nicht mehr lokal, eine Voraussetzung des Modells ist verletzt.

Für eine passive Wand ist aufgrund der Dämpfung der Realteil der Wandadmittanz immer positiv. Die Moden liegen also stets im Bereich der Phase, der in dieser Farbcodierung von blau über grün nach gelb reicht. Bei einer Änderung von $R_{a}$ oder der Dämpfung können die weißen Linien zu $\operatorname{Re}(Y)=0$ nicht gekreuzt werden. Mit abnehmender Dämpfung nähern sich die Moden diesen Linien.

In der hier benutzten Konvention für die Wechselgrößen $\sim \exp \left(-i \omega t+i k_{x} x+i m \phi\right)$ liegen die Moden ohne Strömung, die sich in positiver $x$-Richtung ausbreiten, im ersten Quadranten der $k_{x}$-Ebene, bzw. im Grenzfall ohne Dämpfung auf der positiven reellen und imaginären Achse. Moden, die sich in negativer $x$-Richtung ausbreiten, liegen dagegen im dritten Quadranten bzw. auf den angrenzenden Achsen. In der Abb. 4.1 mit Strömung ist die Phase der Feldadmittanz fast vollständig umgekehrt; die Moden können bei entsprechender passiver Wandadmittanz nahezu überall im 2 . 
und im 4. Quadranten zu liegen kommen. Der Übergang zu diesen Feldadmittanzen erfolgt stetig: Mit wachsender Strömungsgeschwindigkeit schnürren sich die Bereiche im 1. und 3. Quadranten zunehmend zusammen, aus dem Unendlichen nähern sich Bereiche mit entgegengesetzter Phase dem Ursprung. Im 2. und 4. Quadranten dagegen kommen entsprechende Bereiche hinzu, in denen Moden existieren können. Für $\left|k_{x}\right| \lesssim 100 \mathrm{~m}^{-1}$ ist in der Abbildung die ursprüngliche Phase vorhanden. Der Schnittpunkt von $\operatorname{Re}(Y)=0$ mit der reellen Achse für positive $k_{x}$ liegt bei $k_{x}=\omega / \bar{u}$.

\subsubsection{Strömungsprofil}

Mit Strömungsprofil muss die Wellengleichung 4.3 numerisch über den Radius $r$ integriert werden². Als Strömungprofil wird wie bei Großer [10, Kap. 4.3]

$$
\bar{u}(r)=\bar{u}_{0}\left(0,253+0,275 \ln \frac{1,017-r / R}{0,017}\right)
$$

gewählt. Dabei ist $\bar{u}_{0}$ die über den Querschnitt gemittelte Geschwindigkeit der Gleichströmung. Dieses Strömungsprofil weist einen kleineren Sprung an der Rohrwand auf als das Rechteckprofil.

Dieses Strömungsprofil wurde von Rebel und Ronneberger [20] abgeleitet, indem die Konstanten aus experimentellen Daten an das universelle Geschwindigkeitsverteilungsgesetz für große Reynoldszahlen [24, Kap. XX c] angepasst wurden. Es ist demnach ein mittleres Strömungsprofil im Resonatorabschnitt. Die Entwicklung des Strömungsprofils aus dem hartwandigen Rohr zu dem in Gleichung 4.7 gegebenen (und darüber hinaus) wird nicht berücksichtigt.

Abb. 4.2 zeigt die Phase der Feldadmittanz in der $k_{x}$-Ebene für dieses Strömungsprofil bei ansonsten gleichen Parametern wie in Abb. 4.1: $m=0, \mathrm{Ma}=0,175$ und $f=1 \mathrm{kHz}$.

Wie schon beim Rechteckprofil ist auch hier die Feldadmittanz $Y$, auf der reellen $k_{x^{-}}$ Achse rein reell (bis auf den Streifen, an dem die Feldadmittanz einen Sprung aufweist). Die „Blase“ vom Ursprung zu positiven $\operatorname{Re}\left(k_{x}\right)$ hat sich nur wenig verändert. Die Phase von $Y$ hat für positive $\operatorname{Re}\left(k_{x}\right)$ ähnliche Werte wie beim Rechteckprofil. Allerdings tritt außerhalb der „Blase“ bei $\operatorname{Im}\left(k_{x}\right)=0$ für positive $k_{x}$ ein Sprung in der Phase auf. Für reelle $k_{x}$ ist hier die Phasengeschwindigkeit der Welle bei einem Wandabstand (nämlich der kritischen Schicht) gleich der Strömungsgeschwindigkeit. Dort ist $\omega^{\prime}=0$ mit der Folge, dass die Lösungen im verwendeten Modell divergieren. Für noch größere $k_{x}$

${ }^{2}$ In dieser Arbeit wurden stattdessen die Grundgleichungen 4.2 und $4.3 \mathrm{a}$ mit dem harmonischen Ansatz in den Variablen $p$ und $\eta=\frac{i}{\omega^{\prime}} u_{r}$ benutzt und mit der matlab-Funktion ode23s integriert. 


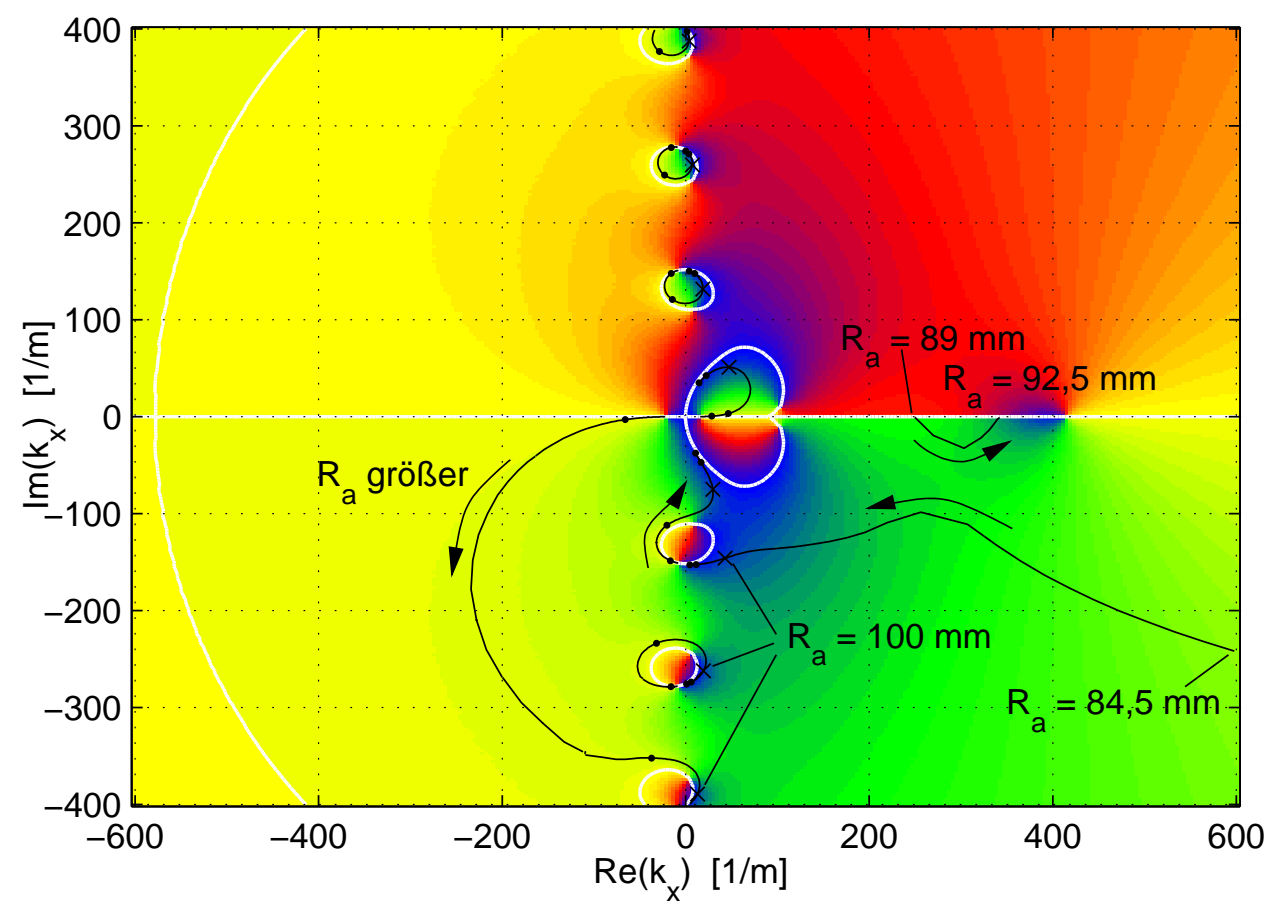

Abb. 4.2: Phase der Feldadmittanz an der Rohrwand, Rechnung mit Strömungsprofil, Auftragung und übrige Parameter wie in Abb. 4.1: $m=0$, $\mathrm{Ma}=0,175$ bei $f=1 \mathrm{kHz}$

verschwindet diese Unstetigkeit wieder - genau dann, wenn die Phasengeschwindigkeit kleiner als die Geschwindigkeit unmittelbar an der Wand ist.

Die Mode, die mit wachsendem $R_{a}$ von der reellen Achse aus erst zu negativen $\operatorname{Re}\left(k_{x}\right)$ anwächst, verläuft hier in wesentlich größerem Bogen. Die von negativen $\operatorname{Re}\left(k_{x}\right)$ ankommende Mode liegt außerhalb des betrachteten Bereichs.

Eine weitere Besonderheit ist die Mode, die an der Unstetigkeit bei $\operatorname{Re}\left(k_{x}\right)=0$ auftritt. Für $R_{a}=89 \mathrm{~mm}$ bis $92,5 \mathrm{~mm}$ bei $k_{x} \approx 300 \mathrm{~m}^{-1}$ kommt sie hervor. Verkleinert man die Frequenz (oder die Dämpfung), so ändert sich auch der Verlauf mit $R_{a}$ als Parameter, die Zweige mit der Mode von großen $\operatorname{Re}\left(k_{x}\right)$ vertauschen: Die Mode, die bei reellem $k_{x}$ entsteht läuft dann zu imaginären $k_{x}$. Die Mode, die von großen $\operatorname{Re}\left(k_{x}\right)$ kommt, mündet dagegen bei reellem $k_{x}$. 


\subsection{Das Problem der Ausbreitungsrichtung der Moden}

\subsubsection{Ausbreitungsrichtung in Vorgängerarbeiten}

Ein besonderes Problem ist es, die Ausbreitungsrichtung der Moden festzustellen. Die Gruppengeschwindigkeit $d \omega / \operatorname{Re}\left(d k_{x}\right)$ eignet sich nicht, wenn eine starke Dämpfung oder Anfachung vorliegt (wie z. B. an evaneszenten Wellen zu sehen ist).

Stattdessen wird in zahlreichen Arbeiten (z. B. Briggs [3], Jones und Morgan [12] $]^{3}$ sowie Crighton und Leppington [7]) die Kreisfrequenz $\omega$ in der komplexen Ebene verändert, so dass die Mode zeitlich angefacht ist, hier also $\operatorname{Im}(\omega)$ groß wird. Wenn die Mode $k_{x}(\omega)$ dabei das Vorzeichen ihres Imaginärteils wechselt, soll die Mode angefacht sein. Dann - so das anschauliche Argument - hat die Anfachung der Quelle die Anfachung durch die Instabilität überschritten.

Ohne ins Detail zu gehen soll dies noch etwas genauer ausgedrückt werden. Der Ausgangspunkt ist, dass die Moden bei einem $\omega$ mit großem Imaginärteil bzw. bei rein imaginärem $\omega$ von der Quelle aus gesehen räumlich abfallen. Mit der hier benutzten Konvention, dass sich die Wechselgrößen $\sim \exp (-i \omega t)$ verhalten, befinden sich dann die stromab laufenden Moden $k_{x}(\omega)$ in der Halbebene mit positiven Imaginärteil, die stromauf laufenden haben ein negatives $\operatorname{Im}\left(k_{x}(\omega)\right)$. Wird $\omega$ nun stetig verändert und so auf die gewünschte reelle Frequenz gebracht, soll sich die Ausbreitungsrichtung nicht ändern. Wenn dabei eine Mode die reelle $k_{x}$-Achse überschreitet und sich damit das Vorzeichen des Imaginärteils ändert, so handelt es sich um eine verstärkte Mode, da nun die konvektive Instabilität die Anfachung durch die Quelle übersteigt.

Nun unterscheiden sich die Ergebnisse in Abhängigkeit des Weges, auf dem $\omega$ variiert wird. So zeigt Rienstra [22], dass es zu falschen Ergebnissen führen kann, wenn bei konstantem Realteil der Imaginärteil von $\omega$ vergrößert wird. Stattdessen schlägt Rienstra vor, den Betrag von $\omega$ konstant zu lassen und die Phase von 0 auf $\pi / 2$ zu ändern.

Für eine solche Änderung von $\omega$ ist der Verlauf der Moden in der $k_{x}$-Ebene in Abb. 4.3 mit grünen Kurven gezeichnet. Hier ist $m=0$ und $\mathrm{Ma}=0,2$, das Strömungsprofil wurde berücksichtigt. Die Lösungen für reelle Frequenzen von 0,3 bis $1,8 \mathrm{kHz}$ sind schwarz eingezeichnet, der Startpunkt bei 0,3 kHz ist mit einem Kreuz gekennzeichnet. Im Abstand von $0,1 \mathrm{kHz}$ ist die Kurve mit Punkten markiert. Für diese markierten Frequenzen wird die Phase von $\omega$ auf $\pi / 2$ gedreht und der durchlaufene Weg grün in die $k_{x}$-Ebene eingezeichnet. In der linken Bildhälfte bzw. im Hintergrund (a) ist ein

\footnotetext{
${ }^{3}$ In der Formulierung von Nilsson und Brander [17]
} 


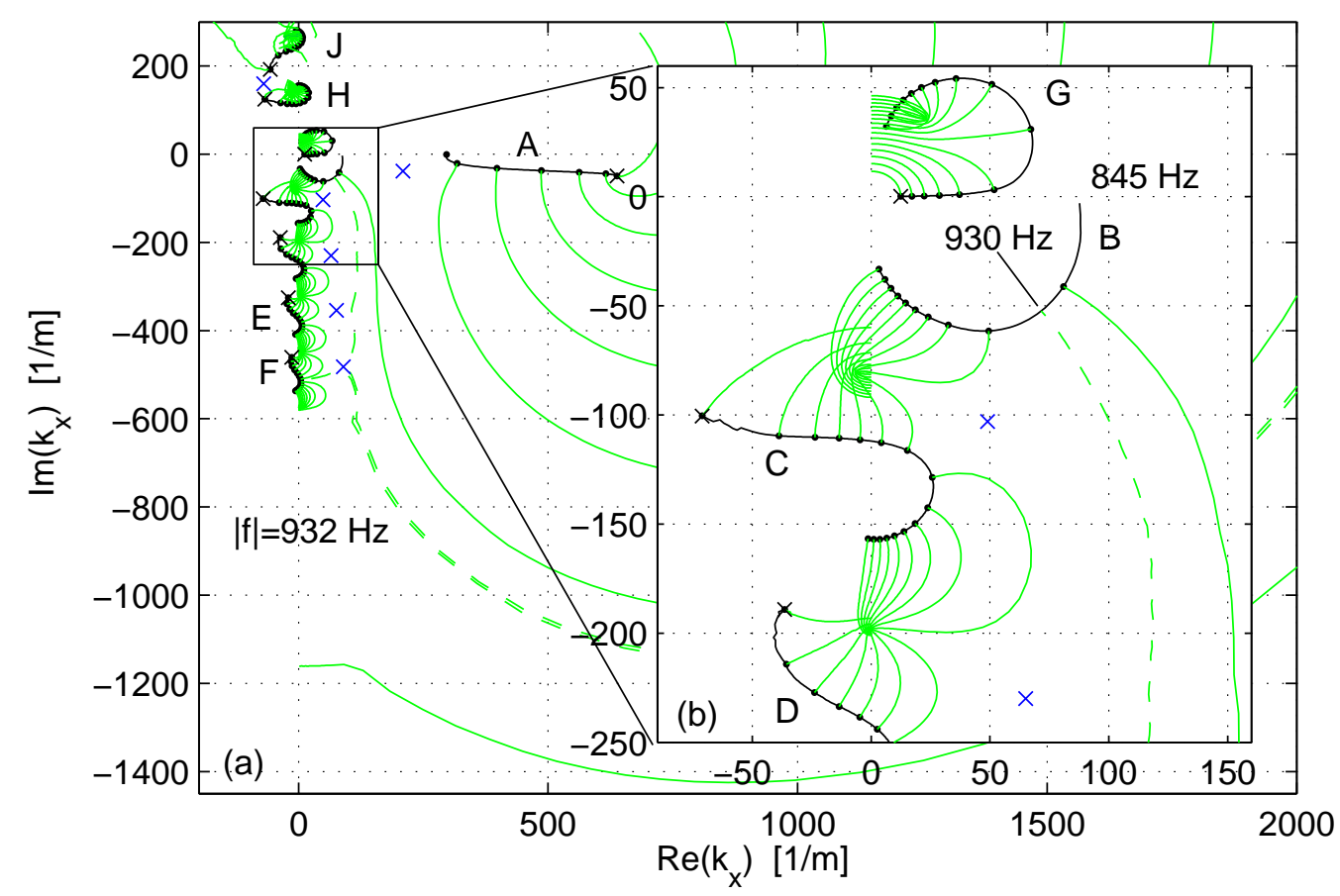

Abb. 4.3: Wellenzahlebene für $m=0$ und $\mathrm{Ma}=0,2$ mit Strömungsprofil, mit vergrößerten Bereich um den Koordinatenursprung. Die schwarzen Linen kennzeichnen reelle Frequenzen zwischen 0,3 und 1,8 kHz. Im Abstand von $0,1 \mathrm{kHz}$ sind Kurven grün gezeichnet, die bei Änderung der Phase von $\omega$ von 0 auf $\pi / 2$ durchlaufen werden. Die blauen Kreuze geben Punkte an, bei denen $d \omega \sim d k^{2}$ ist.

großer Ausschnitt der $k_{x}$-Ebene zu sehen. Hier sind die Moden mit $\operatorname{Im}\left(k_{x}\right)>-500 \mathrm{~m}^{-1}$ eingezeichnet, sowie deren Verlauf bei Änderung der Phase von $\omega$. Rechts (b) ist ein Ausschnitt vergrößert gezeichnet.

Im Verlauf der reellen Frequenzänderung verschwindet die Mode A bei $910 \mathrm{~Hz}$ und $k_{x}=300 \mathrm{~m}^{-1}$, eine neue Mode B kommt bei $845 \mathrm{~Hz}$ und $k_{x}=88 \mathrm{~m}^{-1}$ hinzu. Die übrigen Moden bleiben erhalten.

Der Verlauf der grünen Kurven sollte darüber Aufschluss geben, ob die Mode bei der betrachteten Frequenz instabil ist. Es ist zu erkennen, dass zu jeder Frequenz genau eine Mode bei der Änderung der Phase die reelle $k_{x}$-Achse überschreitet. Für Frequenzen bis $845 \mathrm{~Hz}$ trifft dies auf die Mode A zu. Für Frequenzen bis $930 \mathrm{~Hz}$ ist es die Mode B (gestrichelt eingezeichnet), dann die Mode F (ebenfalls gestrichelt eingezeichnet). Bei noch höheren Frequenzen sind in schneller Folge weitere, nicht eingezeichnete Moden nach diesem Kriterium über einen kleinen Frequenzbereich instabil. Dabei ändert sich 
der Imaginärteil der Moden so, dass sie zunehmend stärker angefacht sein müssten.

Der Wechsel der Mode erfolgt naturgemäß unstetig. Von der Phase $\pi / 2$ kommend sind die Punkte, an denen der Wechsel zwischen dem Endpunkt bei der reellen Frequenz erfolgt, mit blauen Kreuzen in die Abbildung eingetragen. An diesen Verzweigungspunkten ist $d \omega \sim d k_{x}^{2}$, bzw. im Grenzwert des Differentials $d \omega / d k_{x}=0$. Wenn die Änderung von $k_{x}$ nicht exakt entlang der imaginären Achse verläuft, ist damit die Gruppengeschwindigkeit Null, bei einer Frequenz mit positivem Imaginärteil. Damit sind Bedingungen für eine absolute Instabilität erfüllt.

Einige tieffrequente Verzweigungspunkte mit kleinen $\left|k_{x}\right|$ sind in der Tabelle 4.1 aufgelistet. Die Wellenzahlen konnten nicht exakt bestimmt werden, da die Rechnungen numerisch verrauscht sind. Auch in der oberen $k_{x}$-Halbebene kommen solche Verzweigungspunkte vor. Sie treten bereits auf, wenn der Realteil der Frequenz wesentlich tiefer liegt. Im Experiment ist bei diesen Frequenzen kein besonderes Verhalten zu erkennen.

Die Wellenzahl der meisten Verzweigungspunkte hängt nur schwach von der Strömungsgeschwindigkeit ab, da sie zwischen zwei Moden liegen und die Moden sich nur wenig mit der Machzahl verschieben. Nur der mit (*) markierte Verzweigungspunkt liegt nahe $\omega / \bar{u}$, skaliert also mit der Strömungsgeschwindigkeit. Für die meisten Verzweigungspunkte liegt der Realteil der Frequenz unterhalb der im Experiment beobachteten Verstärkung.

Es ist zu erkennen, dass der Weg durch den komplexen $k_{x}-\omega$-Raum entscheidend dafür ist, welche Mode die reelle $k_{x}$-Achse überschreitet. Durch die Erweiterung der $k_{x^{-}}$ Ebene mit reellen $\omega$ zu einem Raum, in dem komplexe $\omega$ zugelassen sind, wird auch die Unterscheidung von getrennten Moden obsolet. Beispielsweise gelangt man von der Mode B nach C, wenn bei der reellen Frequenz $0,9 \mathrm{kHz}$ beginnend die Phase auf $\pi / 2$ gedreht wird, dann die Frequenz im imaginären auf $1 \mathrm{kHz}$ geändert und schließlich die Phase wieder zur rellen Frequenz gedreht wird. Durch ein analoges Vorgehen lassen sich alle gezeigten Moden in der unteren $k_{x}$-Halbebene verbinden.

Bei der Phase der Feldadmittanz, Abb. 4.2, sind Punkte zu sehen, die analog den Verzweigungspunkten sind. Die Phase hat dort einen Sattelpunkt - in der Abbildung beispielsweise bei $k_{x} \approx(300-70 i) \mathrm{m}^{-1}$. Dort kommen sich zwei Moden nahe. Würde die Dämpfung der Wand verringert, so vertauschen die Zweige der Moden. Für eine Frequenzänderung wurde dies oben beschrieben, dabei verändert sich aber die gezeigte Feldadmittanz. Bei einer Änderung der Wandeigenschaft bleibt sie dagegen gleich.

In den Arbeiten von Briggs [3] und Crighton und Leppington [7] wurden Kriterien entwickelt, die eine verstärkte Mode durch Variation der Dispersionsrelation im Komplexen auffinden. Sie betrachten allerdings nur Fälle, in denen die Moden in einem 


\begin{tabular}{|c|c|c|c|c|c|c|c|}
\hline \multicolumn{4}{|c|}{$m=0, \mathrm{Ma}=0,1$} & \multicolumn{4}{|c|}{$m=0, \mathrm{Ma}=0,2$} \\
\hline $\operatorname{Re}(f)$ & $\operatorname{Im}(f)$ & $\operatorname{Re}\left(k_{x}\right)$ & $\operatorname{Im}\left(k_{x}\right)$ & $\operatorname{Re}(f)$ & $\operatorname{Im}(f)$ & $\operatorname{Re}\left(k_{x}\right)$ & $\operatorname{Im}\left(k_{x}\right)$ \\
\hline \multirow[t]{4}{*}{898} & 26 & 49 & 106 & & & & \\
\hline & & & & 339 & 183 & -80 & 435 \\
\hline & & & & 316 & 112 & -80 & 296 \\
\hline & & & & 283 & 25 & -70 & 160 \\
\hline 572 & 68 & -66 & -134 & & & & \\
\hline 621 & 36 & -82 & -266 & & & & \\
\hline 640 & 12 & -87 & -398 & & & & \\
\hline \multirow[t]{2}{*}{ (*) 844} & 4 & 421 & -80 & 845 & 81 & 204 & -38 \\
\hline & & & & 971 & 70 & 49 & -103 \\
\hline 899 & 57 & 63 & -232 & 935 & 220 & 65 & -230 \\
\hline 888 & 35 & 72 & -355 & 889 & 304 & 76 & -354 \\
\hline 875 & 52 & 79 & -480 & 858 & 362 & 90 & -482 \\
\hline 864 & 64 & 85 & -603 & 838 & 411 & 90 & -605 \\
\hline
\end{tabular}

Tabelle 4.1: Frequenz $f[\mathrm{~Hz}]$ und Wellenzahl $k_{x}\left[\mathrm{~m}^{-1}\right]$ einiger Verzweigungspunkte mit positiven $\operatorname{Im}(\omega)$ zu $m=0, \mathrm{Ma}=0,1$ und 0,2 mit Strömungsprofil. Geordnet nach Quadranten, sowie nach $\operatorname{Im}\left(k_{x}\right)$. Der mit $\left(^{*}\right)$ markierte Verzweigungspunkt skaliert mit der Machzahl.

beschränkten Gebiet liegen. In einem abgeschlossenen Raum - wie hier das Rohr existieren dagegen unendlich viele Moden, die in $\operatorname{Im}\left(k_{x}\right)$ nicht beschränkt sind. Daher kann hier ein solches Verfahren vermutlich nicht angewandt werden.

\subsubsection{Weitere Hinweise zur Ausbreitungsrichtung}

Nach meiner Einschätzung ist im vorliegenden Fall die Ausbreitungsrichtung der Moden bisher auf mathematischen Wege nicht sicher festzustellen.

Daher sollen weitere Eigenschaften betrachtet werden, um nach plausiblen Argumenten zur Bestimmung der Ausbreitungsrichtung zu suchen. 


\section{Einordnung in der $k_{x}$-Ebene}

Ohne Strömung und ohne dissipative Effekte liegen die sich nach rechts (stromab) ausbreitenden Moden in der $k_{x}$-Ebene auf der positiven reellen Achse wenn die Mode ausbreitungsfähig ist, ansonsten liegen sie auf der positiven imaginären Achse. Die nach links (stromab) laufenden Moden liegen entsprechend auf den negativen Achsenabschnitten. Unter Berücksichtigung einer Dämpfung im Medium oder in der Wandimpedanz liegen die Moden im ersten bzw. dritten Quadranten.

Demnach sollte eine stromauf angefachte Mode im zweiten, eine stromab angefachte Mode im vierten Quadranten liegen. Wenn die Phasengeschwindigkeit entgegen der Ausbreitungsgeschwindigkeit läuft, könnte eine stromab angefachte Mode auch im dritten Quadranten liegen.

Eine konvektive Instabilität breitet sich typischerweise mit einer Geschwindigkeit in der Größenordnung der halben Strömungsgeschwindigkeit aus, dann ist $k_{x} \approx$ $\frac{2 \omega}{\bar{u}}$, eine akustische Welle höchstens mit der konvektierten Schallgeschwindigkeit. Wenn die Phasengeschwindigkeit gleich der Ausbreitungsgeschwindigkeit ist, sollte $\operatorname{Re}\left(k_{x}\right) \gtrless \pm \frac{\omega}{c} \frac{1}{1 \pm \text { Ma }}$ gelten.

\section{Verhalten bei Parameteränderung}

Um die Ausbreitungsrichtung festzustellen, wurde bereits die Änderung der Frequenz in der komplexen Ebene diskutiert. Ebenso können weitere Parameter geändert werden. Sie sollen nun im Hinblick auf die Ausbreitungsrichtung betrachtet werden. Wie auch bei den komplexen Frequenzen sollte eine stetige Änderung eines Parameters zu einer stetigen Änderung der Mode führen. Wenn von einer bekannten Lösung ausgegangen wird, kann dies als ein Argument für eine Ausbreitungsrichtung genommen werden.

Stetige Parameter sind neben der Frequenz und der Resonatortiefe noch die Dämpfung und die Strömungsgeschwindigkeit. Als diskreter Parameter kommt die azimutale Wellenzahl $m$ hinzu. Auf Änderungen am Strömungsprofil wird nicht eingegangen.

Es ist nicht zu erwarten, dass eine Mode für einen begrenzten Parameterbereich die reelle Achse in $k_{x}$ überschreitet: die Wandadmittanz nach Gleichung 2.4 besitzt immer einen Realteil ungleich Null. Für ein reelles $k_{x}$ ist die Wellengleichung rein reell, die damit berechnete Feldadmittanz folglich rein imaginär, weshalb die Moden der rellen $k_{x}$-Achse nicht beliebig nahe kommen können (siehe Großer [10]). 
Allerdings können mit Strömungsprofil neue Moden hinzukommen, vgl. Abb. 4.2. Diese können sich möglicherweise so verhalten, wie es aus dem Experiment für eine instabile Mode zu erwarten wäre.

\section{Resonatortiefe}

Das Verhalten bei einer Änderung der Resonatortiefe $R_{a}$ ist in der obigen Abb. 4.1 für das Rechteckprofil und in Abb. 4.2 für das realistische Strömungsprofil zu sehen. Mit wachsendem $R_{a}$ kommen beim Rechteckprofil zwei Moden, bei dem realistischen Strömungsprofil eine Mode von großen $k_{x}$ in den betrachteten Bereich. Bei einem hartwandigen Rohr ohne Resonatoren ist $R_{a}=R$. An den Startpunkten der Moden sind also alle Moden stabil, es gibt keinen Mechanismus, der zu einer Instabilität führen kann. Da sich ihre Lage nur relativ wenig verschiebt, sollten es auch stabile Moden bleiben - ausgenommen die von großen $k_{x}$ kommenden Moden, über deren Stabilität nichts gesagt werden kann; sie sind Kandidaten für eine Instabilität. Allerdings münden sie in der Antiresonanz wieder nahe den bekannten stabilen Moden. Auf diesem Weg müsste eine instabile Mode ihre Ausbreitungsrichtung umgekehrt haben.

Mit Strömungsprofil läuft nur eine Mode mit wachsendem $R_{a}$ in den betrachteten Bereich. Eine weitere entsteht an der reellen Achse, wenn $R_{a}$ zwischen 89 und 92,5 mm ist. Um die Schallverstärkung aus dem Experiment erklären zu können, sind diese Radien zu klein. Sie liegen um das Maximum der Wandadmittanz $Y_{W}$. Die Frequenz ist in der Auftragung so gewählt, dass $R_{a} \approx 100 \mathrm{~mm}$ sein müsste.

\section{Dämpfung}

Wird die Dämpfung, also der Realteil der Wandadmittanz, auf Null verkleinert, müssen im Grenzwert die schwarzen Kurven in Abb. 4.1 und in Abb. 4.2 auf den weißen zu liegen kommen. Bemerkenswert daran ist, dass sich mit abnehmender Dämpfung die von großen $\operatorname{Re}\left(k_{x}\right)$ kommende Mode der imaginären Achse nähert. Falls es sich um eine instabile Mode handelt, so nimmt die Anfachung $a b$, wenn die Dämpfung verringert wird. Das spricht gegen eine instabile Welle.

Mit dem Strömungsprofil ist das Verhalten ähnlich, mit Ausnahme des kurzen Abschnitts, der an der Unstetigkeit hervortritt. Dieser entfernt sich mit verringerter Dämpfung zunächst von der Achse und trifft bei $\operatorname{Im}\left(k_{x}\right)=60 \mathrm{~m}^{-1}$ mit der benachbarten Mode zusammen. Bei einer weiteren Verringerung der Dämpfung trennen sich die ursprünglichen Moden am Treffpunkt auf und verschmelzen mit den Zweigen der Nachbarmode. Bei verschwindender Dämpfung kommen sie schließlich auch auf der weiß eingezeichneten Kontur zu liegen. 


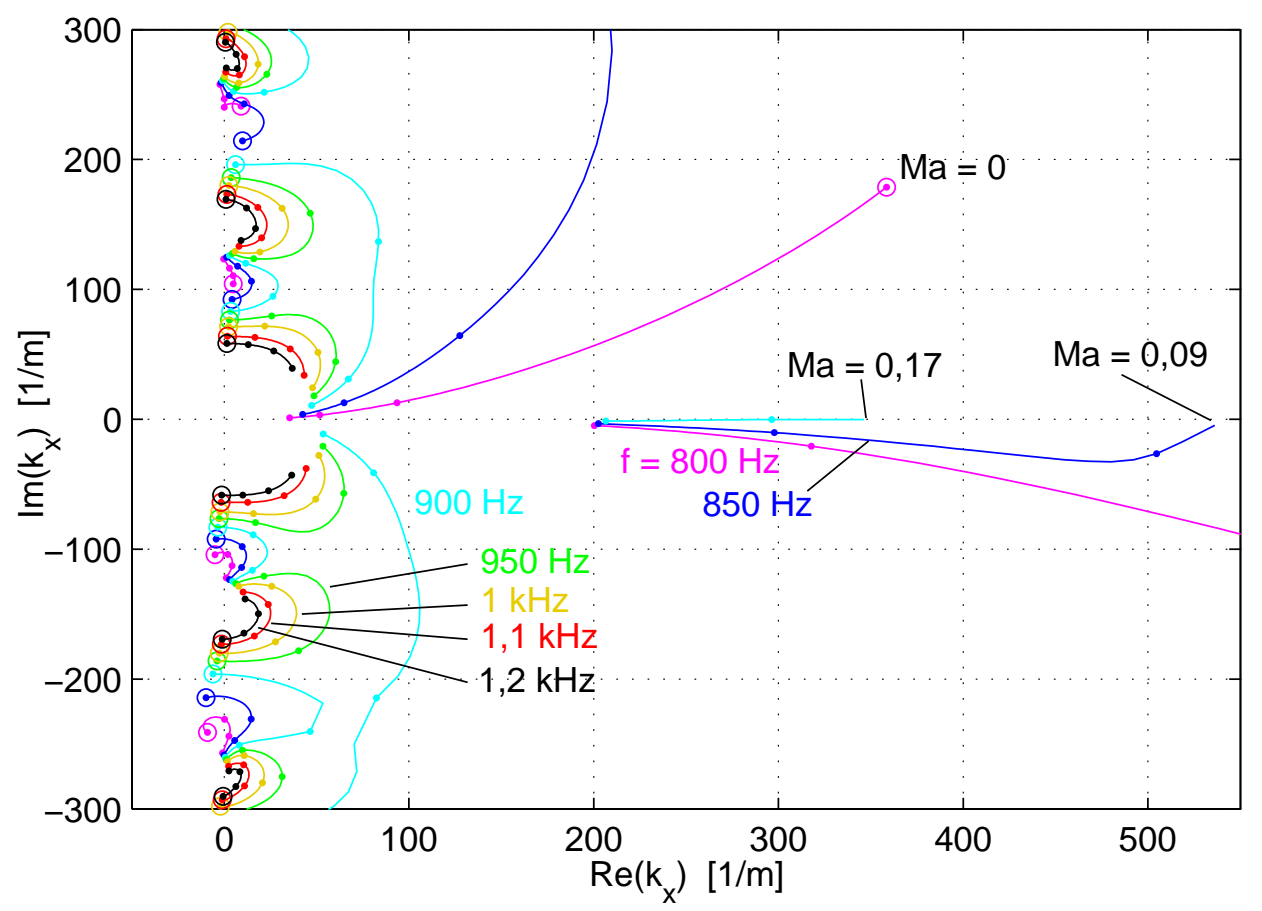

Abb. 4.4: Verlauf der Moden in der $k_{x}$-Ebene für $m=0$ mit Strömungsprofil bei Änderung der Machzahl von $\mathrm{Ma}=0$ (Kreise) bis $\mathrm{Ma}=0,3$, für verschiedene Frequenzen. Punkte markieren die Moden zu Ma =0,1, 0,2 und 0,3.

Im dritten Quadranten liegt eine Mode, deren negative Imatinärteile mit abnehmender Dämpfung größer werden. Der Resonatorradius $R_{a}$ hat dabei allerdings Werte, die keiner im Experiment ausgezeichneten Frequenz entsprechen, das Verhalten tritt deutlich unterhalb der Resonanz auf.

\section{Strömungsgeschwindigkeit}

Wenn keine Strömung vorhanden ist, so können nur gedämpfte Moden existieren. Abb. 4.4 zeigt den Verlauf der Moden in der $k_{x}$-Ebene für ebene Moden $m=0$, die von ruhender Luft (mit Kreisen gekennzeichnet) bis Ma $=0,3$ verfolgt werden. Die Frequenzen liegen zwischen 0,8 und $1,2 \mathrm{kHz}$, also um dem Bereich, in dem im Experiment die Instabilität auftritt. Für noch größere Frequenzen ändert sich die Lage der Moden zunächst nur wenig.

Für die Frequenzen bis $900 \mathrm{~Hz}$ entsteht an der reellen Achse eine Mode, wenn eine zugehörige Machzahl überschritten wird. Da sie ihren Ausgangspunkt nicht in stabilen Moden hat, könnte es sich um verstärkte Mode handeln. Das experimentelle Verhalten kann damit jedoch nicht erklärt werden, da diese Moden nur für Frequenzen bis $900 \mathrm{~Hz}$ 
auftreten. Wenn es sich um die verstärkte Mode handelt, ist auch die Abhängigkeit von der Strömungsgeschwindigkeit anders als im Experiment beobachtet. Mit steigender Machzahl nimmt die Verstärkung ab (bis auf einen kurzen Bereich, an dem die Mode beginnt).

Die übrigen Moden lassen sich von ihrem Startpunkt bei ruhender Luft verfolgen. Im ersten Quadranten nähern sich mit zunehmender Machzahl für 800 und $850 \mathrm{~Hz}$ stark gedämpfte Moden der reellen Achse, wobei auch ihr Realteil kleiner wird. Sie werden nahezu ungedämpft ausbreitungsfähig. Für Frequenzen ab $900 \mathrm{~Hz}$ behalten die angrenzenden Moden auch für $\mathrm{Ma}=0,3$ einen zunehmend größeren Imaginärteil. Allerdings kommen diese Moden ab $900 \mathrm{~Hz}$ Moden mit annähernd konjugiert komplexen Wellenzahlen entgegen - ein Verhalten, das im Folgenden noch öfters bei Frequenzen auftreten wird, bei denen nach dem Experiment eine Instabilität zu erwarten ist. Ein formales Argument, warum es sich hier um instabile Moden handeln sollte, ist jedoch nicht ersichtlich; ohne Strömung haben die Wellenzahlen dieser dann evaneszenten Moden entgegengesetzte Vorzeichen.

Für alle dargestellten Moden ab $950 \mathrm{~Hz}$ nähert sich der Imaginärteil mit steigender Machzahl der reellen Achse. Wenn eine instabile Mode vorhanden ist, würde sie demnach zu größeren Machzahlen schwächer angefacht sein.

Für $m=1$ ist in Abb. 4.5 die Lage der Moden um die Resonanz bei 1,1 kHz dargestellt. Die Moden wurden wie bei der vorigen Abbildung von ruhender Luft ausgehend (mit einem Kreis gekennzeichnet) verfolgt. Die betrachteten Frequenzen liegen zwischen 1,0 und $1,3 \mathrm{kHz}$. Der Verlauf der Moden bei ruhender Luft in diesem Frequenzbereich ist gestrichelt gezeichnet.

Auch hier treten von großen $\operatorname{Re}\left(k_{x}\right)$ mit steigender Machzahl Moden in den betrachteten Bereich, deren Imaginärteil sich dabei der imaginären Achse nähert. Ab der Frequenz, an der eine Instabilität einsetzen sollte, kommen diese Moden nicht mehr vor. Bei wenig höheren Frequenzen liegen sich um den Koordinatenursprung wieder näherungsweise konjugiert komplexe Moden gegenüber.

Schließlich soll zur Änderung der Strömungsgeschwindigkeit noch der Verlauf von tieffrequenten Moden in der $k_{x}$-Ebene betrachtet werden, in Abb. 4.6a ist er für $m=1$ und Frequenzen von 100 bis $700 \mathrm{~Hz}$ dargestellt. Die Machzahl reicht wieder bis 0,3. Zudem ist die Lage einer Mode mit $m=2$ bei $400 \mathrm{~Hz}$ eingezeichnet. Die übrigen Moden mit $m=2$ verlaufen für tiefe Frequenzen nahezu deckungsgleich auf dieser Kurve. Die verschiedenen Frequenzen unterscheiden sich hauptsächlich darin, an welchem Punkt entlang der Kurve eine Mode bei einer gegebenen Machzahl zu liegen kommt.

Die Moden liegen symmetrisch zur reellen Achse. Mit steigender Machzahl laufen sie zunächst auf die Winkelhalbierende im ersten bzw. vierten Quadranten zu und nähern 


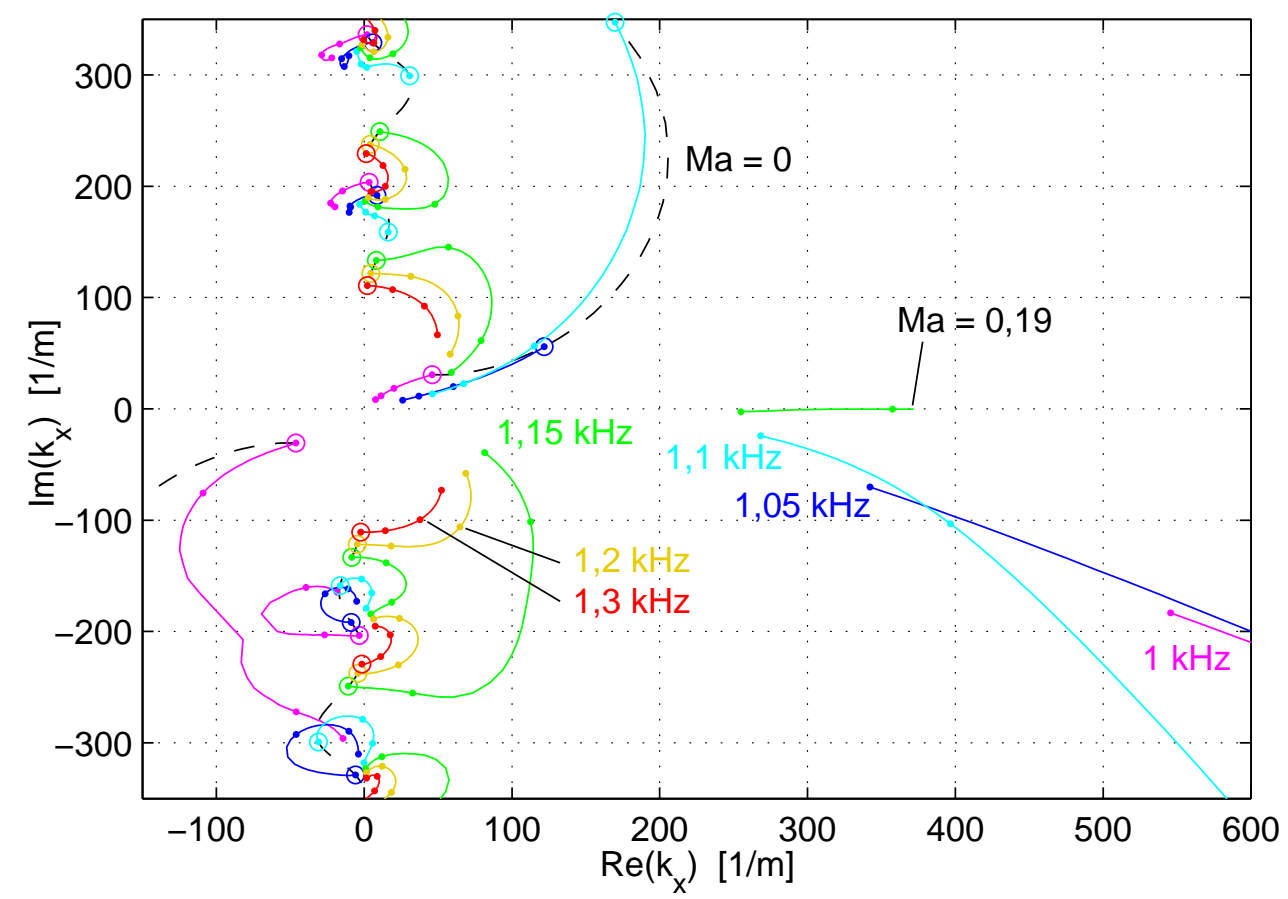

Abb. 4.5: Verlauf der Moden in der $k_{x}$-Ebene für $m=1$ mit Strömungsprofil bei Änderung der Machzahl von $\mathrm{Ma}=0$ (Kreise) bis $\mathrm{Ma}=0,3$ mit Frequenzen zwischen 1 und 1,3 kHz. Punkte markieren die Moden zu Ma =0,1, 0,2 und 0,3. Gestrichelt ist der Verlauf der Moden ohne Strömung von 1 bis $1,3 \mathrm{kHz}$ eingezeichnet.

sich dann $k_{x}=0$. Bei tiefen Frequenzen ändert sich die Wellenzahl stärker mit der Machzahl.

Im Experiment ist sowohl in der Phase turbulent angeregter Druckschwankungen (vgl. Abb. 2.26) als auch bei der Anregung der Mode $m=1$ bei tiefen Frequenzen (vgl. Abb. 2.29) ein ausgezeichnetes Verhalten für Frequenzen entlang der Geraden $f=$ $\mathrm{Ma} \cdot 2,2 \mathrm{kHz}$ zu beobachten. Abb. 4.6b stellt den Verlauf von Moden entlang dieser und ähnlicher Geraden von $f=\mathrm{Ma} \cdot 0,55 \mathrm{kHz}$ bis $f=\mathrm{Ma} \cdot 3,3 \mathrm{kHz}$ dar, wiederum für $m=1$ (durchgezogene Linie) und für die gleichen Verläufe mit $m=2$ (gepunktet). Die Kurven beginnen bei $\mathrm{Ma}=0,001$, also einer sehr kleinen Strömungsgeschwindigkeit. Ein Grenzwert, dem die Moden bei Ma $\rightarrow 0$ möglicherweise zustreben, wird hier nicht erreicht. Insgesamt ändern sich die Wellenzahlen der Moden nur wenig entlang der Parameterkombinationen.

Mit $m=1$ liegt die Instabilität im Experiment entlang $f=\mathrm{Ma} \cdot 2,2 \mathrm{kHz}$. Diese Kurve ist in Abb. 4.6b grün eingezeichnet. Von den benachbarten Moden unterscheidet sie sich 

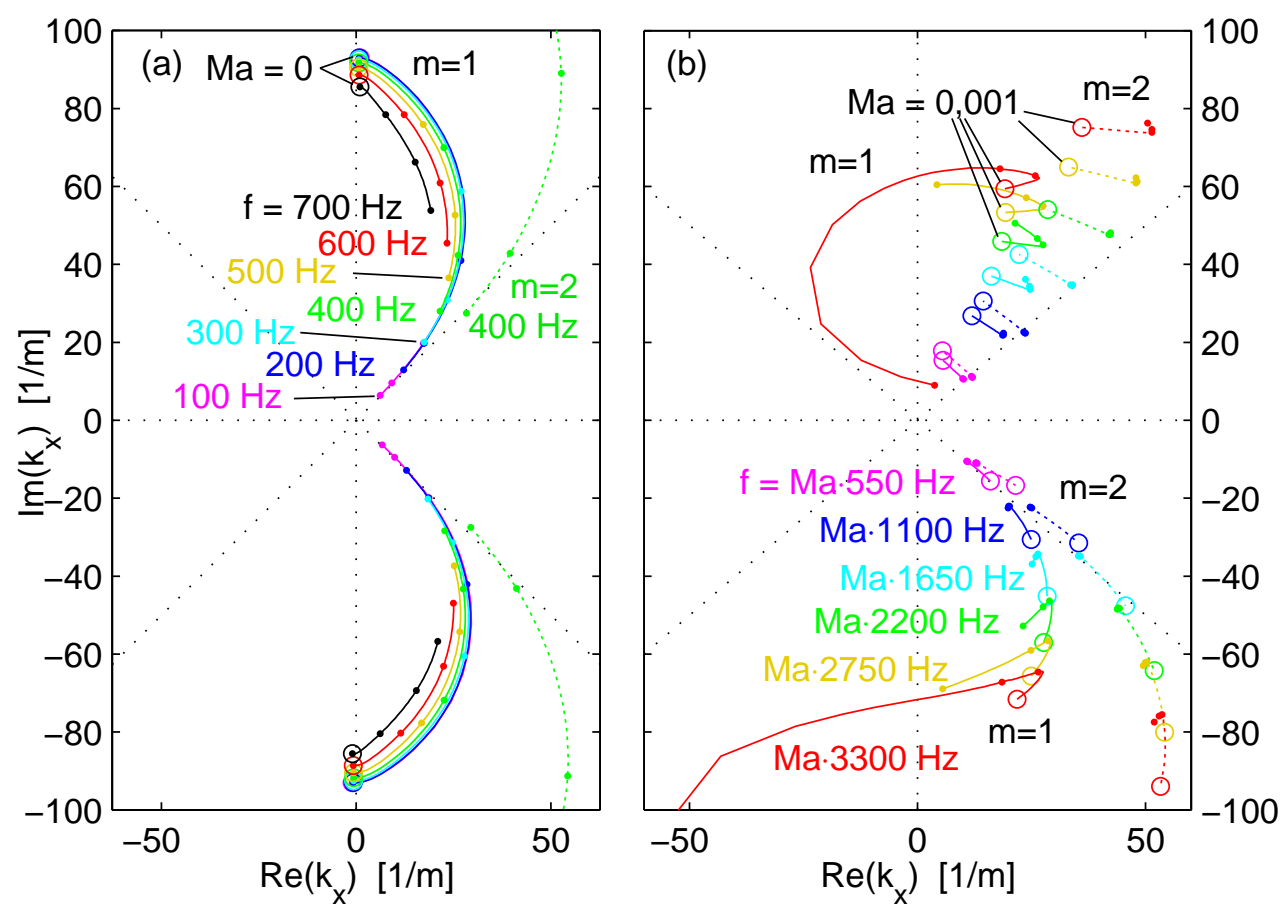

Abb. 4.6: Verlauf der Moden in der $k_{x}$-Ebene für $m=1$ (durchgezogene) und $m=2$ (gepunktete Linie) mit Strömungsprofil bei Änderung der Machzahl von $\mathrm{Ma} \approx 0$ (Kreise) bis $\mathrm{Ma}=0,3$, (a) bei den angegebenen Frequenzen. (b) bei Änderung von Frequenz $f$ und Machzahl für $f \sim$ Ma mit verschiedenen Steigungen. Punkte markieren die Moden zu Ma $=0,1,0,2$ und 0,3.

durch den größeren Realteil, wenn Ma $\lesssim 0,3$. Dagegen wird mit $m=2$ der maximale Realteil bei $f=\mathrm{Ma} \cdot 3,3 \mathrm{kHz}$ erreicht, ohne dass im Phasenspektrum entlang dieser Linie ein ausgezeichnetes Verhalten zu erkennen wäre, vgl. Abb. 2.26.

\section{Frequenz}

Als letzter Parameter wird die reelle Frequenz betrachtet. Der Verlauf der Moden zu $m=0$ in der $k_{x}$-Ebene ist in Abb. 4.7 für Frequenzen von 0,3 bis 1,5 kHz aufgetragen. Frequenzen bis $0,8 \mathrm{kHz}$, also deutlich unterhalb des verstärkten Bereichs, sind mit einer gepunkteten Linie gezeichnet. Kreise markieren die Moden zu $1 \mathrm{kHz}$. Mit einer schwarzen gestrichelten Linie ist der Verlauf der Moden für $0,8 \mathrm{kHz}$ für die Machzahl$\ddot{A}$ derung von $\mathrm{Ma}=0$ bis 0,3 eingetragen.

Bei tiefen Frequenzen beginnend laufen von rechts Moden in den vierten Quadranten der $k_{x}$-Ebene. Bei Frequenzen von ca. $900 \mathrm{~Hz}$ enden sie an der reellen Achse. Bei diese Moden könnten es sich um konvektierte, gedämpfte, stromauf laufende Moden 


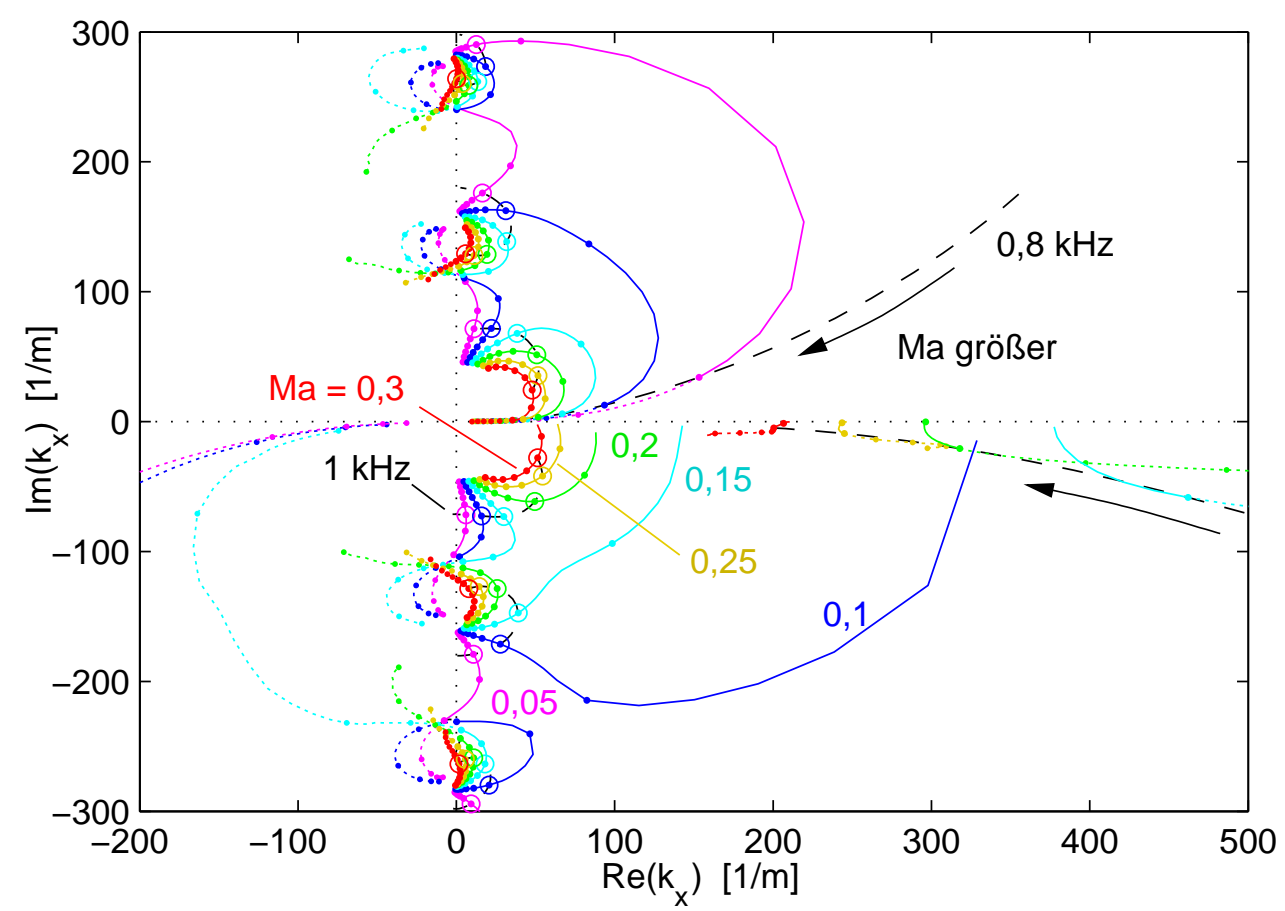

Abb. 4.7: Moden in der $k_{x}$-Ebene für $m=0$ mit Strömungsprofil als Funktion der Frequenz mit der Machzahl als Parameter. Die Frequenz läuft von 0,3 bis $1,5 \mathrm{kHz}$, unterhalb $0,8 \mathrm{kHz}$ ist der Verlauf gepunktet gezeichnet. Punkte markieren die Kurven im Abstand von $0,1 \mathrm{kHz}$, die Moden zu $1 \mathrm{kHz}$ sind mit Kreisen gekennzeichnet.

handeln. Das Verhalten der Wellenzahlen ohne Strömung ist bekannt: Die gedämpften Moden liegen im dritten Quadranten bzw. auf den angrenzenden Achsen. Im Grenzwert tiefer Frequenzen liegt die am schwächsten gedämpfte Mode bei $k_{x 0}^{-}=-\frac{1}{4} \omega / c$, wobei der Faktor 1/4 durch das Volumen der Resonatorkammern zustande kommt, das hier wie eine erhöhte Kompressibilität der Luft wirkt. Wenn sich die Frequenz der Resonanz nähert, läuft die Mode in der Wellenzahlebene nach außen. Schließlich führt eine Dämpfung dazu, dass die Mode durch den dritten Quadranten von einem Punkt nahe der reellen Achse zu einer stark gedämpften Mode auf der imaginären Achse läuft. Noch bevor sich die Mode der imaginären Achse nähert, soll der Effekt betrachtet werden, den eine einfache Konvektion verursacht. Die Phasengeschwindigkeit ist $c_{\mathrm{ph}}:=\omega / k_{x 0}^{-}$. Bei einer überlagerten Strömung mit rechteckigem Strömungsprofil wird daraus $c_{\mathrm{ph}}+$ Ma $c$. Wenn die Strömungsgeschwindigkeit also größer als die Phasengeschwindigkeit ist, verschiebt sich die Mode in den vierten Quadranten, in dem auch die potentiell verstärkten Moden liegen. 


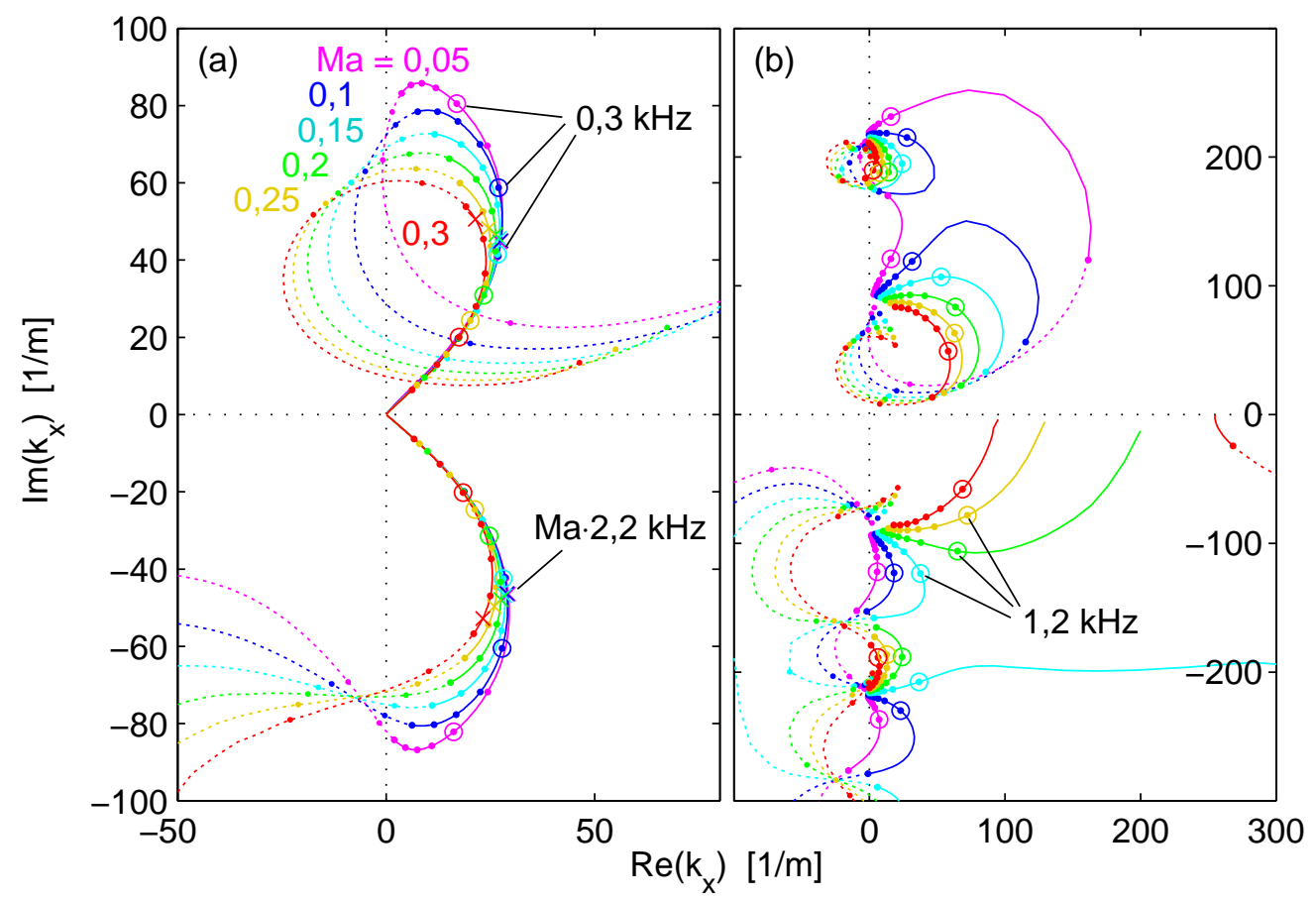

Abb. 4.8: Darstellung wie Abb. 4.7, nur für $m=1$ und geänderten Frequenzen; (a) links: Frequenzen bis $1,1 \mathrm{kHz}$, oberhalb von $0,7 \mathrm{kHz}$ gepunktet, (b) rechts: von 0,7 bis $1,8 \mathrm{kHz}$, unterhalb von $1,1 \mathrm{kHz}$ gepunktet.

Bei etwas tieferen Frequenzen um $850 \mathrm{~Hz}$ entstehen an der reellen Achse Moden mit kleinerem Realteil. Mit steigenden Frequenzen werden sie den konjugiert komplexen Moden ähnlicher, die für alle Frequenzen im ersten Quadranten liegen. Werden die Moden mit Rechteckprofil berechnet, so gehen die zuvor beschriebenen Moden in diese stetig über. Dann entstehen und verschwinden keine Moden bei der Frequenzänderung und der negative Imaginärteil wird kontinuierlich größer.

Aus Abb. 4.7 lässt sich die Gruppengeschwindigkeit $d \omega / d k_{x}$ bzw. $d \omega / \operatorname{Re}\left(d k_{x}\right)$ abschätzen. Wenn die Kurve parallel zur reellen Achse verläuft, sind die beiden Werte gleich und reell. Dies kommt beispielsweise für Ma $=0,2$ mit $f=1 \mathrm{kHz}$ bei den Moden um $k_{x}=(50 \pm 50 i) \mathrm{m}^{-1}$ vor. Mit steigendem $\omega$ wird $\operatorname{Re}\left(k_{x}\right)$ kleiner, die Gruppengeschwindigkeit ist also negativ. Wenn sie mit der Ausbreitungsrichtung übereinstimmen würde, müssten diese stromauf laufenden Moden beträchtlich angefacht sein. Auch bei den anderen Machzahlen ist die Gruppengeschwindigkeit stets stromauf gerichtet, wenn auch der Imaginärteil teils größer als der Realteil ist. Aus den experimentellen Daten wird hier eine stromab laufende Mode erwartet.

Abb. 4.8 zeigt den Verlauf der Moden mit der Frequenz für $m=1$. In Abb. 4.8a ist 
der Frequenzbereich von 0,0 bis $1,1 \mathrm{kHz}$ aufgetragen, mit Kreisen sind die Frequenzen zu $0,3 \mathrm{kHz}$ gekennzeichnet. Kreuze markieren die Moden entlang der erwarteten Verstärkung $f=\mathrm{Ma} \cdot 2,2 \mathrm{kHz}$, vgl. Abb. 4.6b. Die Moden zu den höheren Frequenzen sind in Abb. 4.8b dargestellt, die Frequenz reicht hier bis 1,8 kHz. Kreise markieren die Moden zu 1,2 kHz. In beiden Teilbildern sind die Frequenzen zwischen 0,7 und $1,1 \mathrm{kHz}$, in denen nach dem Experiment keine Instabilität zu erwarten ist, gepunktet aufgetragen.

Tiefe Frequenzen beginnen mit einer positiven Gruppengeschwindigkeit im ersten sowie im vierten Quadranten. Für die mit Kreuzen markierten Frequenzen in Abb. 4.8a wird $d \omega / d k$ nahezu rein imaginär. Nach dem Startpunkt der Mode im vierten Quadranten bei $k_{x}=0$ könnte dies eine instabile Mode sein. Wenn jedoch die Machzahl verringert wird, läuft sie auf eine stabile Mode zu.

\subsection{Zusammenfassung der Modenausbreitung}

Das Verhalten der Moden lässt keinen eindeutigen Schluss über deren Ausbreitungsrichtung zu.

Für eine exponentiell anwachsende Mode spricht, dass immer, wenn eine solche Mode nach dem Experiment erwartet wird, eine entsprechende Mode in der $k_{x}$-Ebene im vierten Quadranten liegt. Diese Mode hat das „richtige“ Vorzeichen der Phasengeschwindigkeit und das "richtige" Vorzeichen der Anfachungsrate. Sie wechselt teilweise das Vorzeichen der Anfachungsrate, wenn die Frequenz ins komplexe erweitert wird, und sich entsprechend einer starken Anfachung der Quelle ändert.

Mehrere Eigenschaften sprechen gegen die Existenz einer verstärkten Mode. So kann eine ins komplexe erweiterte Frequenz derart geändert werden, dass sich die Moden nahezu beliebig ineinander überführen lassen. Damit sind die Moden nicht mehr sinnvoll zu unterscheiden. Wenn eine verstärkte Mode vorhanden ist, so würde sich bei einer Erhöhung der Dissipation in den Resonatorkammern die Anfachung der Mode erhöhen, was zumindest der Intuition widerspricht. Mit steigender Strömungsgeschwindigkeit würde die Anfachung abnehmen, was entgegen dem im Experiment beobachteten Verhalten für $m=0$ steht. Die Frequenzabhängigkeit zeigt kein ausgezeichnetes Verhalten um den Verstärkungsbereich, es kann nicht erklärt werden, warum gerade diese und keine anderen Frequenzen verstärkt werden.

Durch einfache Parameteränderungen können die Moden so verschoben werden, dass eine Verstärkung ausgeschlossen werden kann und es sich um evaneszente Moden handelt. Dieser Übergang erfolgt kontinuierlich, es ist nicht zu erkennen, an welcher Stelle 
sich die Ausbreitungsrichtung ändert. Es ist daher zweifelhaft, ob überhaupt eine mit diesem Ansatz berechnete, exponentiell anwachsende Mode vorhanden ist.

\subsubsection{Alternativer Ansatz}

In den linearisierten Gleichungen sind die Wechselgrößen sehr klein gegen die zeitlich gemittelten Größen. An der Stelle, an der das Strömungsprofil unstetig ist, wurde in Anlehnung an Großer [10] neben dem Druck die radiale Auslenkung $\eta$ als stetige Größe angenommen.

Der Schall wird als infinitesimale Änderung betrachtet, die den zeitlich gemittelten Gleichkomponenten überlagert ist. Im Modell weist die Gleichströmung $\bar{u}(r)$ an der Stelle $r=R$ einen Sprung auf, der in einer mikroskopischen Betrachtung nicht vorhanden ist. Er wird durch die viskose Unterschicht gebildet. Im bisherigen Modell wird vorausgesetzt, dass die viskose Unterschicht groß gegenüber der radialen Auslenkung des Schallfeldes ist.

Die Auslenkung soll für den radialsymmetrischen Fall $(m=0)$ bei $1 \mathrm{kHz}$ abgeschätzt werden: Im Rohr liegt der Schalldruck etwa bei 50 Pa (siehe Abb. A.1). Nach Abb. 2.4 liegt er im Resonator um den Faktor 7 darüber, also bei 3,5 Promille des statischen Drucks. Um den Druck im Resonator statisch um diesen Wert zu erhöhen, muss die Luft von $R=25 \mathrm{~mm}$ um $\sqrt{R_{a}^{2}-\left(R_{a}^{2}-R^{2}\right) / 1,0035}-R=0,65 \mathrm{~mm}$ ausgelenkt werden. Die Auslenkung ist damit nicht klein gegen die viskose Unterschicht. Wenn der Schalldruck bis zur letzten Resonatorkammer um den Faktor 10 angestiegen ist, reicht die Auslenkung schon beträchtlich in das Rohr, selbst wenn die Resonanzüberhöhung mit der Strömung kleiner ist.

In jedem Fall sollte in einem künftigen Modell berücksichtigt werden, dass die Gleichströmung sich mit dem Schallfeld in radialer Richtung verschiebt. Wenn sie in Richtung der Resonatorkammern ausgelenkt ist, gibt sie axialen Impuls durch Reibung und Druckfelder an die Resonatorkammern ab. Wenn diese Impulsabgabe „phasenrichtig“ zur axialen Schallschnelle ist, kann damit möglicherweise sowohl die Schallverstärkung als auch der Druckverlust erklärt werden. 


\section{Kapitel 5}

\section{Schlussbetrachtungen}

\subsection{Ergebnisse}

In dieser Arbeit wurde eine strömungsakustische Instabilität untersucht, die in einem Rohr auftritt, in dem ein Abschnitt der Rohrwand aus gleichartigen, scheibenförmigen Resonatorkammern besteht. Die betrachtete Instabilität tritt auf, wenn die Wandadmittanz groß ist, also bei der Resonanz der Kammern. Die Frequenz größter Instabilität liegt meistens etwas oberhalb der Resonanzfrequenz.

Regt man die Instabilität durch eine ebene Schallwelle an, so kann sich der Druckabfall an dem Resonatorabschnitt in etwa verdoppeln. Dies führt allerdings zu einer starken Schallabstrahlung am Auslauf des Resonatorabschnitts.

Hier wurde erstmals gezeigt, dass Instabilitätswellen auch mit Wechselfeldern angeregt werden können, die keine ebenen Moden sind. Speziell wurde im runden Rohr die Mode mit der Umfangswellenzahl $m=1$ angeregt, mit der der Druckabfall fast ebenso effektiv gesteuert werden kann wie im axialsymmetrischen Fall. Diese Anregung bietet den Vorteil, dass die Wechselfelder auf den Resonatorabschnitt beschränkt bleiben, dass also kein Schall abgestrahlt wird. Die erste Resonanz der Kammern liegt bei dieser Anregung mit 1,11 kHz etwas höher als die axialsymmetrische Resonanz. Auch der Frequenzbereich, bei dem durch die Anregung von azimutalen Instabilitäten ein erhöhter Druckabfall auftritt, ist nach oben verschoben. Es sind zwei Maxima unabhängig von der Machzahl bei $1,2 \mathrm{kHz}$ und bei $1,3 \mathrm{kHz}$ zu beobachten.

In einem weiteren, ursprünglich nicht erwarteten Bereich tiefer Frequenzen kann der Druckabfall ebenfalls durch die Anregung einer azimutalen Instabilitätswelle erhöht werden. Die Frequenzen wachsen proportional zur Strömungsgeschwindigkeit. Auch hier ist die Wandadmittanz groß, wird aber mit wachsenden Frequenzen kleiner. Der 
erzielbare Druckabfall nimmt mit steigender Strömungsgeschwindigkeit (also zu größeren Frequenzen) ab. Für die verschiedenen Strömungsgeschwindigkeiten ändert sich der Frequenzverlauf des Druckabfalls recht stark. Bei einem ringförmigen Kanalquerschnitt nimmt der erreichbare Druckabfall mit der Strömungsgeschwindigkeit schneller ab, als beim kreisförmigen Querschnitt.

Im Rechteckkanal wurden zwei verschiedene Resonatorabschnitte betrachtet. Einmal befinden sich scheibenförmige Resonatoren an einer Kanalwand, im zweiten Aufbau befinden sie sich an zwei gegenüberliegenden Kanalwänden. Mit der ersten Anordnung ist aufgrund der Geometrie keine gegenphasige Anregung möglich. Schallverstärkung tritt um die 1. Resonanz ab Ma = 0,25 auf. Obwohl sich das Spektrum der turbulenten Druckschwankungen zwischen diesen Machzahlen deutlich ändert, sind die Änderungen im Transmissionsfaktor stetig. In der 2. Resonanz tritt die Schallverstärkung schon ab $\mathrm{Ma}=0,20$ auf und ist etwas stärker. Auch hier treten die Überhöhungen im Durckspektrum bei den gleichen Frequenzen auf, aber die Stärke der Überhöhung lässt nicht unmittelbar auf die Stärke des Transmissionsfaktors schließen.

Wenn zwei Kanalwände mit Resonatoren bestückt sind, so tritt bei Frequenzen um die 1. Resonanz bis $\mathrm{Ma}=0,25$ keine Schallverstärkung auf, obwohl im Spektrum eine Überhöhung zu sehen ist. Die Signale an den gegenüberliegenden, stromabseitigen Resonatorkammern sind nur schwach korreliert. Dagegen tritt um die 2. Resonanz ab $\mathrm{Ma}=0,2$ Schallverstärkung auf. Hier sind die Signale in den gegenüberliegenden Kammern stärker korreliert, allerdings sind die Signale überwiegend gegenphasig. Der erzielbare Druckabfall ist unabhängig davon, ob die Instabilität an beiden Wänden gleich- oder gegenphasig angeregt wird. Bei kleinen Machzahlen liegt der stärkste Druckabfall um die 1. Resonanz, mit wachsender Machzahl wird der Druckabfall kleiner. Im Gegenzug wächst der Druckabfall um die 2. Resonanz bis Ma =0,25 an, dort wird dann ein größerer Effekt als in der 1. Resonanz erzielt.

Der Druckabfall, der durch eine Anregung von Frequenzen im Instabilitätsbereich zustande kommt, verhält sich in Abhängigkeit von der Anregungsamplitude für alle Geometrien und Anregungsarten qualitativ ähnlich. Bei kleinen Amplituden tritt zunächst kein Effekt auf. Bei größeren Amplituden steigt der Druckabfall am Resonatorabschnitt linear an, wenn die Amplitude exponentiell erhöht wird.

Wenn die Anregung den Druckabfall am Resonatorabschnitt verändert, ist auch das Wechseldruckspektrum beeinflusst, das im Kanal stromab des Resonatorabschnitts gemessen wird. Im Rechteckkanal sind die spektralen Überhöhungen relativ schmalbandig, der Schalleinfluss auf das Spektrum wurde nur für den runden Abschnitt gezeigt, da er in diesem Fall besser zu sehen ist. Ohne zusätzliche Schallanregung werden die turbulenten Druckschwankungen in einem gewissen Bereich verstärkt. Mit einer 
monofrequenten Anregung nehmen die übrigen Spektralkomponenten zugunsten der eingebrachten Frequenz ab. Wie weit sie abnehmen, hängt von der Lage der Frequenz im verstärkten Bereich und von der Anregungsstärke ab.

Die Nichtlinearitäten, die im Druckabfall und im Wechseldruckspektrum deutlich werden, hängen möglicherweise ursächlich zusammen. Der erhöhte Druckabfall kommt durch einen vermehrten Transport von axialem Impuls zur Kanalwand zustande, der sich auch in einem geänderten mittleren Strömungsprofil bemerkbar macht. Dadurch wird die Verteilung der Turbulenz beeinflusst, die für den Impulstransport verantwortlich ist. Die Umwandlung der Turbulenz in Schall am Auslauf des Resonatorabschnitts ist damit von der Anregungsfrequenz und vom Druckabfall abhängig.

\subsection{Ausblick}

Das Auftreten der betrachteten Instabilität stellt sich in den verschiedenen Geometrien und Parameterbereichen äußerst komplex dar. Es erscheint nicht möglich, für ihr Auftreten einfache Forderungen an die Wandimpedanz und die Strömungsgeschwindigkeit zu stellen. Auch komplexere Ansätze, die die Geometrie des Kanals einbeziehen, waren bisher nicht erfolgreich (siehe Kapitel 4 in dieser Arbeit, Großer [10] und Rienstra [21], [22]).

Es ist noch immer unklar, welcher Mechanismus zu der Instabilitätswelle führt. Zur Klärung sind weitere, insbesondere theoretische Untersuchungen nötig. Diese sollten über die zitierten Ansätze hinausgehen. Vermutlich muss die periodische Änderung des lateralen Transports axialen Impulses berücksichtigt werden. In einer relativ einfachen Form lässt sich dieser Implulstransport über die Modulation der Wandschubspannung beschreiben. Ein solcher Ansatz, in den experimentell bestimmte Parameter eingeflossen sind, wurde für tiefe Frequenzen von Rebel und Ronngeberger benutzt [20].

Aus mathematischer Sicht bleibt die Frage nach den Randbedingungen besonders am Einlauf des Resonatorabschnitts interessant. Physikalisch formuliert wäre dies die Frage, welche Größe für die Anregung der Instabilitätswelle entscheidend ist, wie also die Instabilität besonders effektiv angeregt oder im Gegenzug unterdrückt werden kann.

Im Hinblick auf eine Anwendung als schallgesteuertes Ventil wäre eine Untersuchung von längeren Resonatorabschnitten von Interesse. Dabei stellt sich die Frage, wie die Sättigung des Druckabfalls verläuft. Möglicherweise setzt die globale Instabilität bei kleineren Strömungsgeschwindigkeiten ein und wird damit durch die Anregung weniger beeinflussbar. Es ist aber auch denkbar, dass eine angeregte Mode über den gesamten Resonatorabschnitt bestehen bleibt und so in langen Abschnitten der Druckabfall 
noch weit stärker beeinflusst werden kann. In diesem Zusammenhang wäre auch die Reaktionszeit von Interesse, die zwischen der Anregung und dem Druckabfall benötigt wird. 


\section{Anhang A}

\section{Ansteuerungsparameter}

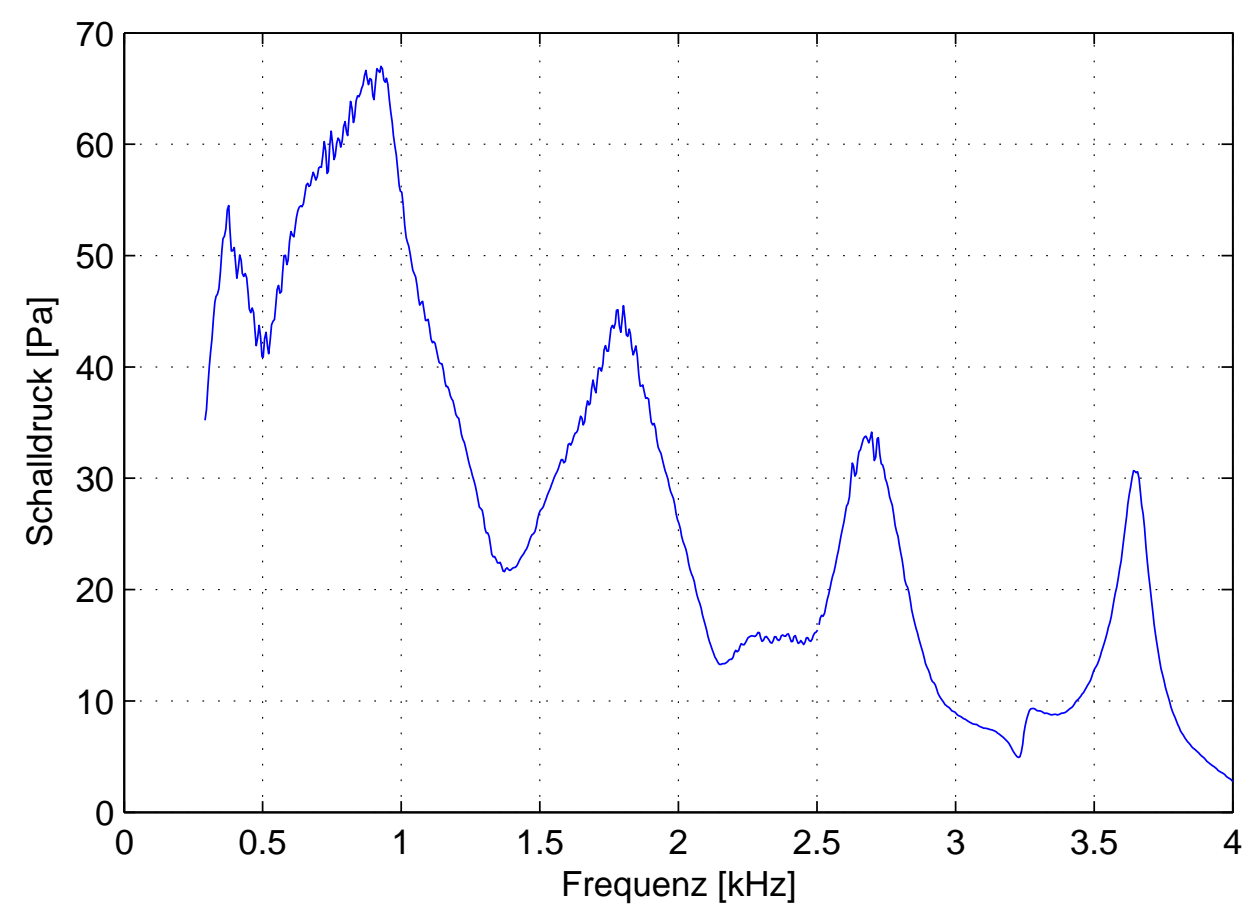

Abb. A.1: Frequenzabhängigkeit des Lautsprechers im leeren Messrohr unter der Annahme, dass die Mikrofonempfindlichkeit konstant bleibt. 


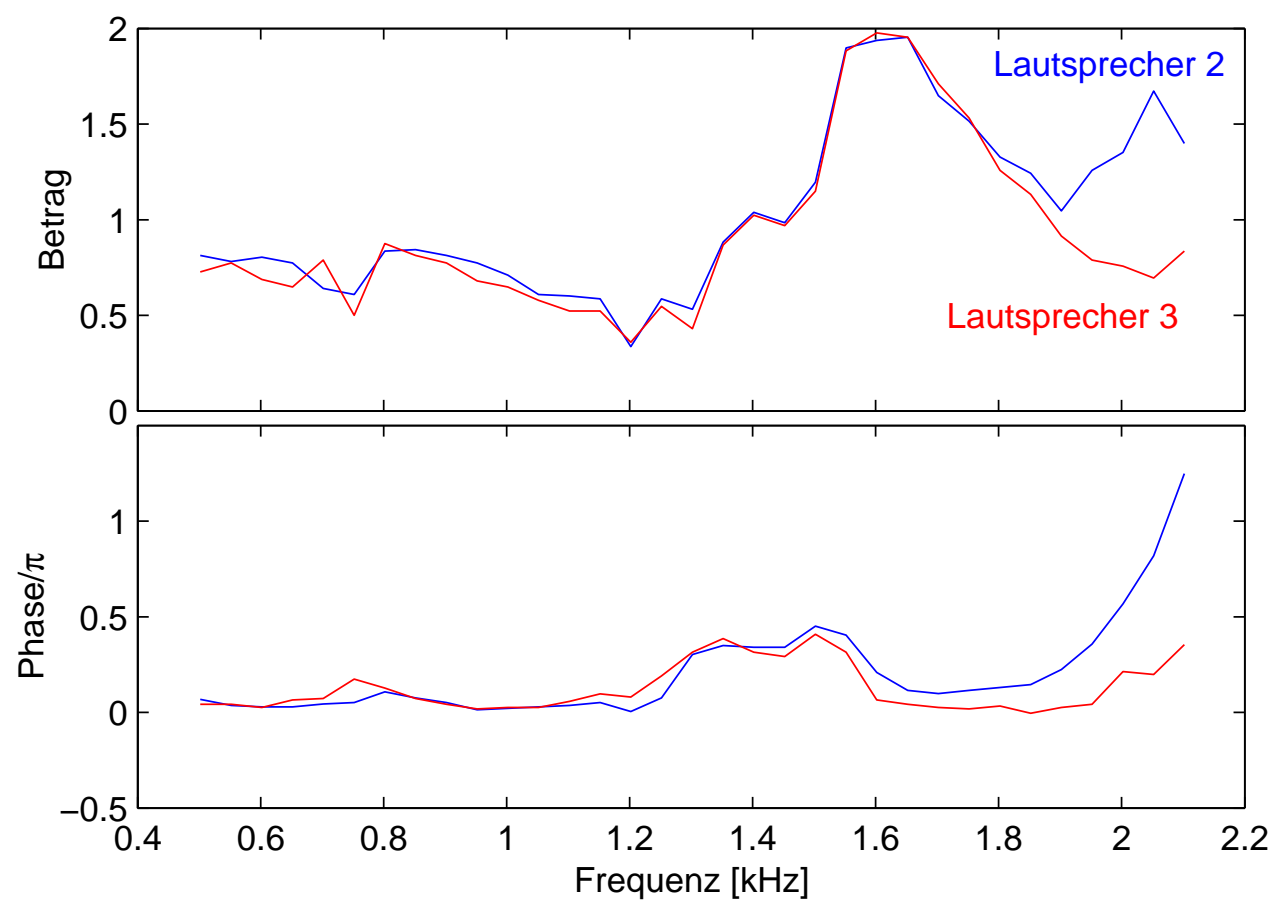

Abb. A.2: Ansteuerungsparamter für die beiden Kompensationslautsprecher, bezogen auf den Referenzlautsprecher 1 , hier für $\mathrm{Ma}=0,25$ gemessen. Der Betrag kann maximal mit 2 angesteuert werden. 


\section{Literaturverzeichnis}

[1] M. Brandes: Optimierung eines Meßverfahrens zur Bestimmung von akustischen Parametern im durchströmten Rohr. Diplomarbeit, Universität Göttingen, 1992.

[2] M. Brandes: Schallverstärkung in Strömungskanälen mit resonanzartiger Wandauskleidung. Dissertation, Universität Göttingen, 1997.

[3] R. J. Briggs: Electron-Stream Interaction with Plasma. Res. Monogr. No. 29, MIT press, Cambridge Massachusetts, 1964.

[4] I. N. Bronstein, K. A. Semendjajew, G. Musiol und G. Mühlig: Taschenbuch der Mathematik. Harri Deutsch Verlag, Frankfurt a. M., 1995.

[5] O. Cramer: The variation of the specific heat ratio and the speed of sound in air with temperature, pressure, humidity, and $\mathrm{CO}_{2}$ concentration. JASA 93, S. 2510-2516, 1993.

[6] L. Cremer: Die wissenschaftlichen Grundlagen der Raumakustik, III. Wellentheoretische Raumakustik. Hirzel, 1950.

[7] D. G. Crighton und F. G. Leppington: Radiation properties of the semi-infinite vortex sheet: the initial-value problem. J. Fluid Mech. 64, S. 393-414, 1974.

[8] L. Enghardt: Bestimmung akustischer Streufaktoren im durchströmten Rohr: Optimierung von Mikrofonanordnung und Meßprozedur. Diplomarbeit, Universität Göttingen, 1992.

[9] S. Förster: Einfluß der Wandschubspannung auf die Schallausbreitung in durchströmten Kanälen mit porösen Wänden. Diplomarbeit, Universität Göttingen, 1987.

[10] J. Großer: Modellbildung für die Schallverstärkung in nachgiebig ausgekleideten Strömungskanälen. Dissertation, Universität Göttingen, 2003. 
[11] P. Huerre und P. A. Monkewitz: Local and global instabilities in spatially developing flows. Annual Review of Fluid Mechanics 22, S. 473-537, 1990.

[12] D. S. Jones und J. D. Morgan: A linear model of a finite amplitude helmholtz instability. Proc R. Soc. Lond. A. 338, S. 17-41, 1974.

[13] W. Koch und W. Möhring: Eigensolutions for liners in uniform mean flow ducts. AIAA Journal 21, S. 200-213, 1983.

[14] M. Krause: Schallausbreitung in Strömungskanälen mit kassettierter Wandauskleidung. Diplomarbeit, Universität Göttingen, 1990.

[15] B. Lange: Aktive Schalldämmung mittels einer strömungsakustischen Instabilität. Diplomarbeit, Universität Göttingen, 1998.

[16] B. Lange und D. Ronneberger: Active noise control by use of an aeroacoustic instability. Acta Acustica united with Acustica 89, S. 658-665, 2003.

[17] B. Nilsson und O. Brander: The propagation of sound in cylindrical ducts with mean flow and bulk-reacting lining, i. modes in an infinite duct. J. Inst. Maths Applics 26, S. 269-298, 1980.

[18] M. C. Quinn und M. S. Howe: On the production and absorption of sound by lossless liners in the presence of mean flow. Jounal of Sound and Vibration 97, S. $1-9,1984$.

[19] J. Rebel: Experimentelle Untersuchung der Schallstreuung in Strömungskanälen mit Wandimpedanzsprüngen. Diplomarbeit, Universität Göttingen, 1988.

[20] J. Rebel und D. Ronneberger: The effect of shear stress on the propagation and scattering of sound in flow ducts. Jounal of Sound and Vibration 158, S. 469-496, 1992.

[21] S. W. Rienstra: A classification of duct modes based on surface waves. Wave Motion 37, S. 119-135, 2003.

[22] S. W. Rienstra und N. Peake: Modal scattering at an impedance transition in a lined flow duct. 11st AIAA/CEAS Aeroacoustics Conference, 2005.

[23] D. Ronneberger: Abschlußbericht zum DFG-Forschungsvorhaben „Akustische Refle xion und Transmission von Einbauten in durchströmten Kanälen ". Drittes Physik. Institut, Universität Göttingen, 1987.

[24] H. Schlichting: Grenzschichttheorie. G. Braun Verlag, Karlsruhe, 1982. 



\section{Danksagung}

Diese Arbeit wurde am Dritten Physikalischen Institut der Universität Göttingen unter Anleitung von Herrn Prof. Dr. Dirk Ronneberger angefertigt. Bei ihm möchte ich mich für das anhaltende Interesse an der Aufgabenstellung und die Unterstützung über seine Pensionierung hinaus bedanken.

Bei Herrn Prof. Dr. Ulrich Parlitz bedanke ich mich für die Übernahme des Korreferates.

Der mechanischen Werkstatt danke ich für die Anfertigung des rechteckigen Resonatorabschnitts als auch diversen kleineren Zubehörs. Mein Dank gilt auch der elektronischen Werstatt für die Ausführung zahlreicher kleinerer Arbeiten.

Danken möchte ich auch Dr. Arno Ickler für sein Interesse an meiner Arbeit sowie Dr. Christoph Jüschke für das Korrekturlesen. 



\section{Lebenslauf}

Name:

Geburtstag:

Geburtsort:

Staatsangehörigkeit:

$1991-1994$

4. Juli 1994

Juli 1994 - Juli 1995

Sept. 1995 - Juli 2001

Sept. 2001 - Aug. 2004

WS 2001/02 - SS 2006
Matthias Jüschke

31. Oktober 1974

Oldenburg

deutsch

Gymnasium Eversten in Oldenburg

Abitur

Wehrdienst bei der Marine in Wilhelmshaven

Physikstudium an der Georg-August-Universität Göttingen

Diplomarbeit am Dritten Physikalischen Institut unter Anleitung von Prof. Dr. D. Ronneberger

Stipendiat des Graduiertenkollegs „Strömungsinstabilitäten und Turbulenz"

Promotionsstudium an der Georg-August-Universität Göttingen 